DOE $|B C| 14652 \ldots-T \mid$

\title{
DISPERSION MEASUREMENT AS A METHOD OF QUANTIFYING GEOLOGIC CHARACTERIZATION AND DEFINING RESERVOIR HETEROGENEITY
}

Annual Report of the Period

July 12, 1990 - September 12, 1991

\author{
By \\ Donald E. Menzie \\ Principal Investigator
}

April 1992

Work Performed Under Contract No. 22-90BC14652.000

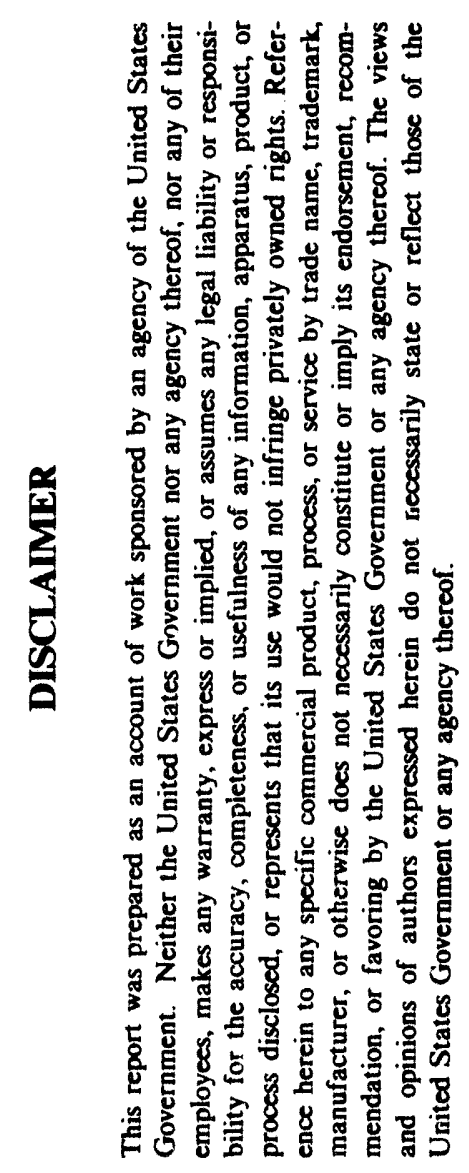

\author{
Prepared for \\ U.S. Department of Energy \\ Assistant Secretary for Fossil Energy \\ Gene Pauling \\ Contracting Officer's Representative \\ U.S. Department of Energy \\ Metairie Site Office \\ 900 Commerce Road, East \\ New Orleans, LA 70123 \\ Prepared by \\ University of Oklahoma \\ Norman, Oklahoma 73019
}




\section{Personnel Involved}

Principal Investigator: Post Doctoral Fellow: Post Graduate: Graduate:

Undergraduate:

\section{Donald E. Menzie}

Reza S. Shadizadeh

S. Chen, Ph.D. Saba Tahmassebi, Ph.D. student; Yan Li, Ph.D. student; Dianli Han, Ph.D. student; Wei Wang, M.S. student; and Yongqing Li, M.S. student

Robert Ho 


\section{TABLE OF CONTENTS}

Page

TABLE OF CONTENTS $\ldots \ldots \ldots \ldots \ldots \ldots \ldots \ldots \ldots$ iii

LIST OF TABLES $\ldots \ldots \ldots \ldots \ldots \ldots \ldots \ldots \ldots \ldots \ldots \ldots$

LIST OF FIGURES $\ldots \ldots \ldots \ldots \ldots \ldots \ldots \ldots \ldots \ldots$ vii

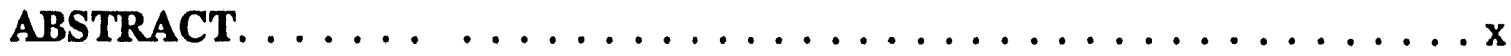

CHAPTER

1. INTRODUCTION $\ldots \ldots \ldots \ldots \ldots \ldots \ldots \ldots \ldots$

1.1 Why Well Logs? . . . . . . . . . . . . . . . . . 1

1.2 Objectives and Scope of the Study $\ldots \ldots \ldots \ldots \ldots \ldots \ldots$

2. LTTERATURE REVIEW $\ldots \ldots \ldots \ldots \ldots \ldots \ldots$

2.1 Occurrence of the Dispersion Phenumena . . . . . . . . . 3

2.2 General Models for Dispersion Phenomena . . . . . . . . . . . 5

2.2.1 Convection Dispersion (or Diffusion-type) Model . . . . . . . 5

2.2.2 Dispersion Capacitance Model . . . . . . . . . . 10

2.2.2.1 Brigham's Solution . . . . . . . . . . . . 10

2.2.2.2 Coats and Smith's Solution . . . . . . . . 12

2.3 Techniques for Determining Dispersivities $\ldots \ldots \ldots \ldots \ldots 14$

2.4 Controlling Factors for Dispersivity . . . . . . . . . . . 17

2.5 Correlations of Dispersivity with Rock Petrophysical Properties . . . 21

2.5.1 Dispersivity Versus Permeability . . . . . . . . . . 21

2.5.2 Dispersivity Versus Other Rock Parameters . . . . . . . 25

2.6 Scale Dependency of Dispersivity ... . . . . . . . . . 25

2.6.1 Arguments on Scale Dependency . . . . . . . . . 25

2.6.2 Hypotheses for Scale Dependency . . . . . . . . . . 30 
3. THEORETICAL DEVELOPMENT OF THE RELATIONSHIP BETWEEN DISPERSIVITY AND LOG-DERIVABLE ROCK PROPERTIES $\ldots \ldots \ldots \ldots \ldots \ldots \ldots \ldots \ldots$

3.1 The Concepts of the Tortuosity and Tortuosity Factor . . . . . . 31

3.1.1 Measurement of Tortuosity ............. 34

3.1.1.1 Fluid Flow Measurement $\ldots \ldots \ldots \ldots \ldots \ldots$

3.1.1.2 Electrical Measurement . . . . . . . . . . . . 36

3.2 Rock Properties in Terms of Tortuosity . . . . . . . . . . . 37

3.2.1 The Tortuous Capillaric Model for a Porous Medium . . . . . 37

3.2.2 Electrical Properties of a Porous Medium . . . . . . . . 38

3.2.2.1 Formation Factor Versus Tortuosity $\ldots \ldots \ldots \ldots 38$

3.2.3 Fluid Flow Properties of a Porous Medium . . . . . . . . 40

3.2.4 Dispersion Process in a Porous Medium . . . . . . . . 42

3.3 Relating Dispersivity to Rock Parameters . . . . . . . . . . . 43

4. DETERMINATION OF ROCK PROPERTIES FROM WELL LOGS 45

4.1 Pre-treatment of Well Log Data $\ldots \ldots \ldots \ldots \ldots \ldots$. . . . . . 45

4.2 Determination of Porosity . . . . . . . . . . . . . 45

4.3 Determination of Formation Factor and Tortuosity . . . . . . 46

4.3.1 Mud Fluid Invasion Profile . . . . . . . . . 46

4.3.2 Absolute Tortuosity From Micro-Log . . . . . . . . . . . 46

4.3.3 Undisturbed Formation Resistivity and Saturation . . . . . . 49

4.4 Estimation of In-Situ Permeability . . . . . . . . . . 51

4.5 Determination of Heterogeneity Factor $\ldots \ldots \ldots \ldots \ldots \ldots 2$

4.5.1 Defining Heterogeneity Factor in Terms of Permeability Variation . . . . . . . . . . . . 52

4.5.2 Determining Permeability Variation Coefficient . . . . . . . 54

5. LABORATORY EXPERIMENTAL DESIGN AND PROCEDURES . . 56

5.1 Preparation of Core Samples $\ldots \ldots \ldots \ldots \ldots \ldots \ldots$

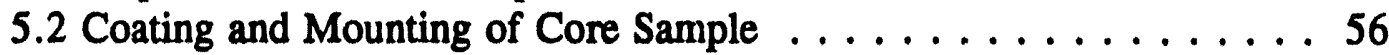

5.3 Preparation of Fluids . . . . . . . . . . . . . . 60

5.3.1 Brine Solution for Electrical Measurement . . . . . . . . 60

5.3.2 Miscible Fluids for Displacement Test . . . . . . . . 60

5.4 Measurements of Basic Rock Properties . . . . . . . . . . . . . 60

5.4.1 Measurement of Porosity, Formation Factor, and Permeability . 63

5.4.1.1 Porosity Measurement . . . . . . . . . . . . . 63

5.4.1.2 Formation Factor and Tortuosity Measurement . . . . 63

5.4.1.3 Preparation for Fluid Flow Tests . . . . . . . 65

5.4.1.4 Fluid Permeability Measurement . . . . . . . . . . 65 
5.5 Miscible Displacement Tests $\ldots \ldots \ldots \ldots \ldots \ldots$. . . . . 68

5.5.1 Equipment and System Setup . . . . . . . . . . . 68

5.5.2 Operation Procedure of the Experiment . . . . . . . . 68

6. CHARACTERIZING ROCK HETEROGENEITIES WITH

DISPERSION MEASUREMENT $\ldots \ldots \ldots \ldots \ldots \ldots \ldots \ldots 71$

6.1 Measurement of Heterogeneity . . . . . . . . . . . . 71

6.2 Correlations of Heterogeneity Factor with Rock Parameters . . . . 75

6.2.1 Heterogeneity Factor Versus Permeability . . . . . . . . 75

6.2.2 Heterogeneity Factor Versus Tortuosity . . . . . . . . . 75

7. CHARACTERIZING RESERVOIR ROCKS WITH DISPERSIVITY . 83

7.1 Dispersion Model for Matching Concentration Profiles $\ldots \ldots \ldots$. . 83

7.1.1 New Definition for the Parameter $f$ in the

Dispersion-Capacitance Model . . . . . . . . . . . . 83

7.1.2 Relationship Between Dispersion Fraction and

Heterogeneity Factor . . . . . . . . . . . . . . . . 84

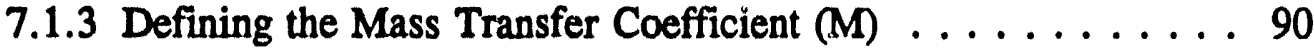

7.2 Determining the Standard Dispersivity . . . . . . . . 92

7.2.1 Matching Concentration Profile . . . . . . . . . . 92

7.2.2 Defining the Equivalent Dispersivity . . . . . . . . 92

7.2.3 Effect of the Multiple Solutions ............. . 95

7.3 Relating the Parameter $\sigma_{\mathrm{H}}$ to the Heterogeneity Factor $\mathrm{H} \ldots \ldots .95$

7.4 Comparison of Laboratory Measured Dispersivity With

Theoretical Dispersivity . . . . . . . . . . . . . 100

8. CONCLUSIONS . . . . . . . . . . . . . . . . . . . . . . 104

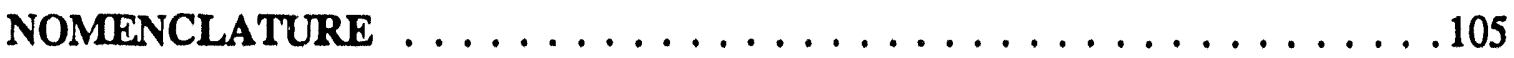

REFERENCES. . . . . . . . . . . . . . . . . . . . . . . 109 


\section{LIST OF TABLES}

Table

Page

2.1 Correlation of Dispersivity with Rock Parameters (after Klotz and Moser, 1974) . . . . . . . . . . . . . . . . . 26

5.1a Description of Core Samples (Group \#1) . . . . . . . . . . . . . 57

$5.1 b$ Description of Core Samples (Group \#2) . . . . . . . . . . . . . . 58

5.1c Description of Core Samples (Group $\# 3) \ldots \ldots$. . . . . . . . . 58

$5.1 \mathrm{~d}$ Description of Core Sarnples (Group \#4) . . . . . . . . . . . . . 59

5.1e Description of Core Samples (Group \#5) . . . . . . . . . . . . . . 59

5.2 Brine Solution Data $\ldots \ldots \ldots \ldots$. . . . . . . . . . . . 62

5.3 Physical Properties of Methanol and Toluene . . . . . . . . . . . . 62

5.4 Measured Petrophysical Properties of Core Samples . . . . . . . . . . . 66

6.1 Elements of Heterogeneity That Influence Fluid Flow in a Reservoir (after Alpay, 1972) . . . . . . . . . . . . . . . 72

6.2 Evaluated Heterogeneity Factors of Core Samples . . . . . . . . . . . . 76

7.1 Fitting Parameters for Matching Concentration Profiles and the Resultant Dispersivities . . . . . . . . . . . . . . . . . 93

7.2 Rock Heterogeneity Factors and Theoretical Dispersivities . . . . . . . . 101

$7.3 \sigma_{H}$ Values for Natural Sandstones . . . . . . . . . . . . . . . . . . 102 
2.1 Solvent concentration profile in an idealized linear miscible displacement (Chen et al., 1984) $\ldots \ldots \ldots \ldots \ldots \ldots \ldots \ldots \ldots$

2.2 Microscopic convective dispersion (Stalkup, 1983) $\ldots \ldots \ldots \ldots \ldots 6$

2.3 Concentration profiles showing capacitance effect

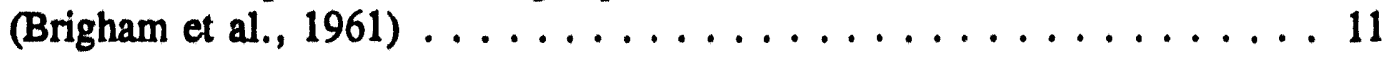

2.4 Effects of the parameters in capacitance model on the shapes of concentration profiles (Batycky et al., 1982) $\ldots \ldots \ldots \ldots \ldots \ldots 15$

2.5 Illustration of the graphical method to determine dispersivity $\ldots \ldots \ldots 18$

2.6 Dispersion coefficient versus peclet number and grain size (Blackwell, 1962) . . . . . . . . . . . . . . . . . . . . . 19

2.7 Correlation between dispersion and particle size (Harleman et al., 1963) . . . . . . . . . . . . . . . . . 22

2.8 Correlation between dispersion and permeability (Harleman et al., 1963) . . . . . . . . . . . . . . . . . 23

2.9 Correlation between dispersivity and permeability (data from Harleman et al., 1963) . . . . . . . . . . . . . . . . . . . . 24

2.10 Laboratory measured dispersivities on sand packs showing

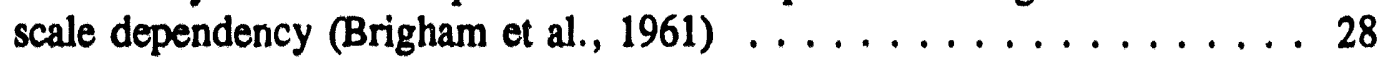

2.11 Field and laboratory dispersivities showing scale-dependency (Lake et al., 1986) . . . . . . . . . . . . . . . . . . . . . . . . 29

3.1 Illustration of a tortuous path in a porous medium (Walsh and Bruce, 1984) 
3.2 Variation of the cross-sectional area of a tortuous path (Winsauer et al., 1952) $\ldots \ldots \ldots \ldots \ldots \ldots \ldots \ldots \ldots$

3.3 Illustration of the electrical double-layer on the interface of solid and fluid phases in a porous medium (Serra, 1984) . . . . . . . 35

3.4 Illustration of the tortuous capillaric model for a porous medium ([a] from Pfannkuch, 1972; [b] from Walsh and Brace, 1984) . . . . . . 39

4.1 Mud fluid invasion profile (Schlumberger, 1991) . . . . . . . . . 47

4.2 Electric log responses in mud fluid invaded formations (Schlumberger, 1990) . . . . . . . . . . . . . . . . . . . 48

4.3 Relationship between heterogeneity factor and Dykstra-Parsons permeability variation (from Koval, 1963) . . . . . . . . . . 53

5.1 "J-B Weld" coated core assembly and cross section (Menzie et al., 1988) 62

5.2 A conductivity bridge apparatus for measuring resistivities $\ldots \ldots \ldots \ldots 6$

5.3 System setup for steady-state miscible displacement test $\ldots \ldots$. . . . 6 69

6.1 Defining heterogeneity factor in terms of solvent cut at one pore volume of solvent injection in a matched viscosity flood . . . . . . 74

6.2 Permeability versus heterogeneity for Group \#1 Berea sandstone samples . 78

6.3 Permeability versus heterogeneity for Group \#2 Berea sandstone samples . 79

6.4 Permeability versus heterogeneity factor for 1.5 " diameter Berea sandstone samples (Groups $\# 3$ and $\# 4) \ldots \ldots \ldots \ldots \ldots$

6.5 Permeability versus heterogeneity for 1 " diameter Brown sandstone samples (Group \#5) $\ldots \ldots \ldots \ldots \ldots \ldots$. . . . . . . . . 81

6.6 Heterogeneity factor as a function of tortuosity in Berea and Brown sandstone samples . . . . . . . . . . . . . . . . 82

7.1 Defining dispersion fraction based on the peak of the first derivative of concentration $\ldots \ldots \ldots \ldots \ldots \ldots \ldots$

7.2 Relationship between dispersion fraction $\left(f_{\alpha}\right)$ and heterogeneity factor $(H), \quad 87$ 
7.3 Dispersion fraction as a function of heteroginneity factor in $1^{\text {" diameter }}$ Berea Samples . . . . . . . . . . . . . . . . . . 88

7.4 Dispersion fraction as a function of heterogeneity factor in 1 " diameter Brown samples $\ldots \ldots \ldots \ldots \ldots \ldots \ldots$. . . . . . . . . 89

7.5 Mass transfer coefficient $(M)$ as a function of stagnant pore fraction in Berea and Brown sandstone samples . . . . . . . . . . . 91

7.6 Effects of dispersion fraction and mass transfer coefficient on the equivalent dispersion coefficient (Baker, 1977) . . . . . . . . . . 94

7.7a Multiple solutions to the capacitance model resulting in almost the same dispersivity $\ldots \ldots \ldots \ldots \ldots \ldots \ldots \ldots$

7.7b Multiple solutions to the capacitance model resulting in almost the same dispersivity . . . . . . . . . . . . . . . . . . 97

7.8 Dispersivity versus heterogeneity factor in Berea and Brown sandstone samples $\ldots \ldots \ldots \ldots \ldots \ldots \ldots$. . . . . . . . 99

7.9 Comparison of laboratory measured dispersivity with theoretical prediction . . . . . . . . . . . . . . . . . 103 


\begin{abstract}
Since reservoirs are heterogeneous, nonuniform, and anisotropic, the success or failure of many enhanced oil recovery techniques rests on our prediction of internal variability and the paths of fluid flow in the reservoir. The main objective of this project is to develop a greater understanding of reservoir heterogeneities through dispersion measurement. In this annual report, an approach to ways to estimate the dispersivities of reservoir rocks from well logs is presented. From a series of rock property measurements and dispersion tests the following studies have been made:

(a) A measure of rock heterogeneity is developed by using the effluent concentration at one pore volume injection in a matched viscosity miscible displacement. By this approach, a heterogeneity factor is determined from the measured S-shaped dispersion curve.

(b) The parameter $f$ in the Coats-Smith capacitance model is redefined as the dispersion fraction $f_{d}$ (or mechanical mixing fraction). At the $f_{d}$ pore volume injection, the dynamic miscible displacement efficiency reaches maximum. Reflected on the dispersion curve, this number corresponds to the peak of the first derivative of concentration.

(c) With the concept of dispersion fraction, a unique solution to the capacitance model is obtained, and then an equivalent dispersivity is defined. Through experimental data on Berea and Brown sandstone samples, it has been found that the equivalent dispersivity is an exponential function of the heterogeneity factor and can be used as a reservoir characteristic.

(d) Through a key parameter of tortuosity, dispersivity is related to rock petrophysical properties. This semi-theoretical relationship forms the basis for determining dispersivities from well logs. The approach is validated through experimental studies on Berea and Brown sandstone samples. It has been found that the equivalent dispersivity is an exponential function of the heterogeneity factor and can be used as a reservoir characteristic.
\end{abstract}




\section{CHAPTER 1}

\section{INTRODUCTION}

A reservoir rock can be conventionally characterized by a variety of macroscopic pore structure parameters such as porosity, permeability, specific surface area, formation resistivity factor, and tortuosity. All of these macroscopic parameters are influenced, to a greater or lesser degree, by the pore structure (Dullien, 1979). Even though there are many sharacteristic parameters available to describe a reservoir rock, it is still difficult to characterize a dispersion process when two miscible fluids flow through the reservoir rock. Thus a new characteristic parameter, dispersivity, is proposed.

By definition, dispersivity is a property that characterizes the mixing ability of a porous rock when miscible fluids flow through it. Theoretically it has been shown that dispersivity is primarily a function of the pore structure of the porous medium. Due to this fact, one of the primary goals in the present study is the characterization of reservoir rocks using the parameter of dispersivity.

\subsection{Why Well Logs ?}

Currently the dispersivity of a reservoir rock is usually determined either by matching a laboratory measured S-shaped concentration profile with a theoretical model or by using Brigham's method of plotting laboratory measured data on an arithmetic probability coordinate paper (Brigham, 1972). Field tracer tests are often precluded due to the excessive expense and time involved. Tests on core samples (plugs) in the laboratory are often impossible because the availability of cores is very limited. Practically, very few wells are usually cored because of high expenses and the time involved. To obtain the field-wide dispersivity values of the reservoir rock, a core-independent technique of determining dispersivities using other available sources of information must be developed.

It is well-known that well logging is an alternative, and very effective, approach to obtain in-situ measurements of rock properties. To date, many different types of well logging tools such as electrical logs, sonic logs, nuclear logs, and mechanical logs have been developed. With these tools, a number of physical parameters that are related to both geological and petrophysical properties of the strata that have been penetrated are measured. This information is sometimes incomplete or disturbed, but always permanent, continuous and objective (Serra, 1984). Traditionally, well logging data are usually used for geological correlation, porosity and hydrocarbon saturation determination. In recent years, well logging measurements have been firmly established 
as having applications in reservoir evaluation and characterization. If the rock dispersivity can be successfully determined from well logs, the pivotal role of well logs in reservoir characterization can be enhanced.

In general, the major advantages of using well logging data rather than core analysis of dispersivity determination are the following:

(a) well logging provides in-situ information about the reservoir rocks, which eliminates the effects of inappropriately simulated underground conditions and the disturbance caused by the coring process;

(b) well logging provides a larger scale of measurements, which results in a better representation of rock heterogeneities;

(c) well logging provides unbiased and relatively uniform quality data from well to well, even field to field;

(d) well logs are extensive throughout the entire reservoir and continuous along the total depth of the borehole because almost all wells drilled for petroleum exploration and development, even dry holes, are logged; and

(e) well logs are very cost effective and time saving.

\subsection{Objectives and Scope of the Study}

The objectives of the work presented in this annual report are three-fold: (1) to investigate the potential of using the parameter of dispersivity to characterize reservoir rocks, (2) to evaluate the rock heterogeneities with dispersion measurement, and (3) to develop a method of estimating the dispersivities of reservoir rocks from well logs. 


\section{CHAPTER 2}

\section{LITERATURE REVIEW}

The object of this chapter is to describe the basic dispersion phenomena, to review dispersivity studies involved in the miscible displacement processes in porous media and to put in perspective the various contributions to this subject.

Because of the potential applications of dispersivity in various fields, the subject of the dispersion phenomena has received not only the attention of petroleum industry researchers, but also of civil, chemical, hydrology, physics, and environmental industry researchers. Over the past several decades, a great amount of literature has been published about this subject ranging from theoretical analysis, mathematical formulation, model development, computer modeling, and experimental studies, to field tracer tests. Since the literature on this subject is so voluminous, only the papers that are most pertinent to the scope of this work are presented in this section.

\subsection{Occurrence of the Dispersion Phenomena}

When two miscible fluids are in contact, with one fluid being displaced by another, dispersion occurs. A mixing zone will develop with a resultant S-shaped concentration profile. This process is illustrated in Figure 2.1 where different S-shaped curves are observed at different times and different locations inside the medium. This phenomenon was first reported by Slichter (1905) who used an electrolyte as a tracer in studying ground water movement. In his experiment, Slichter noticed that the tracer's concentration increases gradually at the observation point, and that even in a uniform fluid field, the tracer moves in the direction of the flow in a pear-like form which becomes longer and wider as it advances.

Many mechanisms are responsible for the occurrence of dispersion. Some of them described by Greenkorn and Kessler (1970) are (a) molecular diffusion, (b) eddy migration, (c) tortuosity, (d) connectivity, (e) flow restriction and recirculation, (f) deadend pores, (g) adsorption and (h) adherence. Bear (1969) described the mechanisms as: (a) external forces acting on the liquids, (b) the microscopic intricate geometry of the pore system, (c) variations in liquid properties such as density and viscosity which affect the flow pattern, (d) changes of the displacing fluid's concentration due to chemical and physical processes within the liquid phase, and (e) interactions between the fluid and the solid phases. Generally, three distinct mechanisms are believed to contribute to the growth of the mixing zone: molecular diffusion, microscopic convective dispersion, and macroscopic convective dispersion. Molecular diffusion is the transport of mass because 


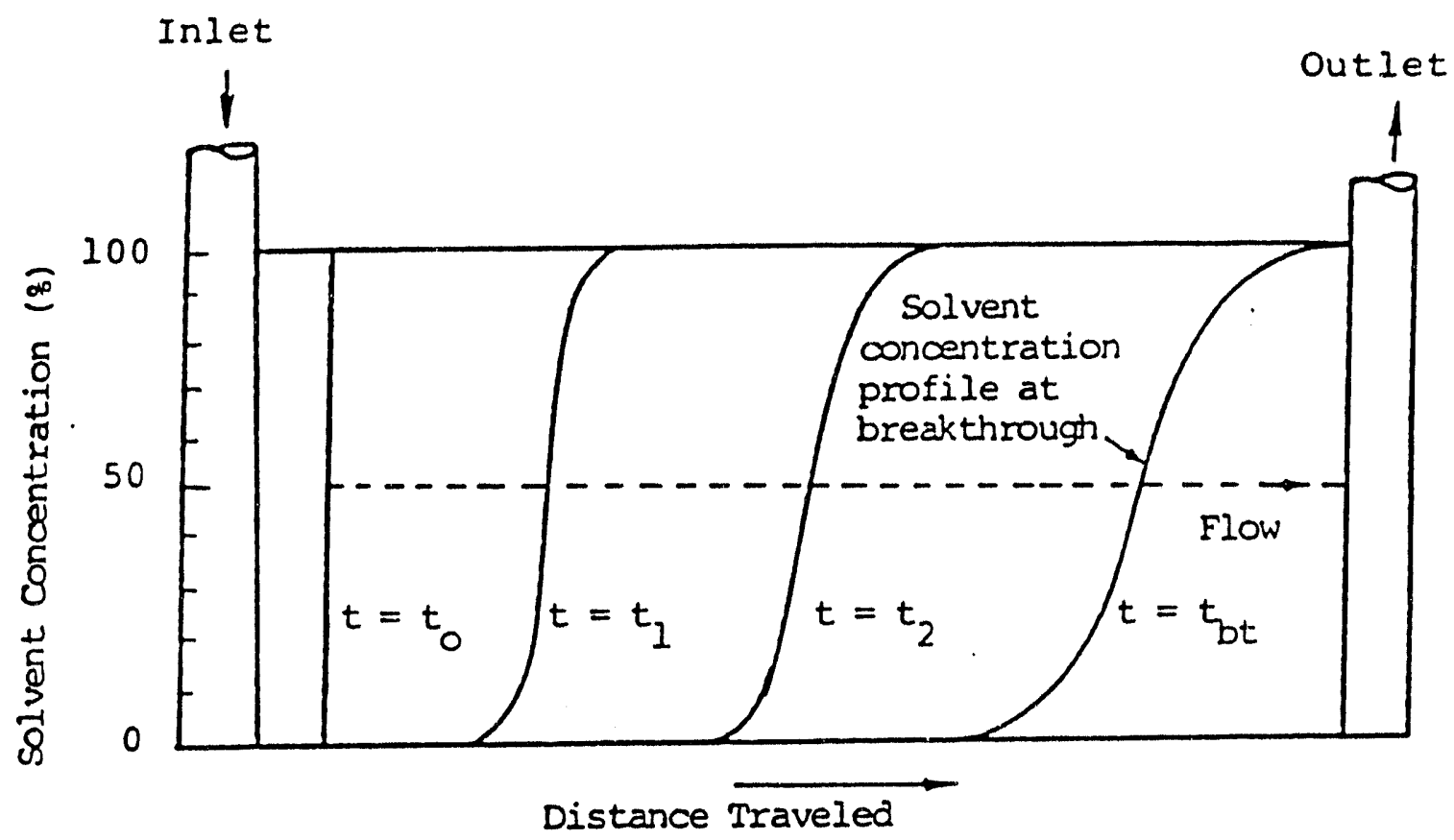

Figure 2.1: Solvent concentration profile in an idealized linear miscible displacement (Chen et al., 1984) 
of spatial concentration differences. Macroscopic convective dispersion results from the flow paths caused by permeability variation, while microscopic convective dispersion is mixing caused by variations in the microscopic velocity within each flow channel and from one channel to another. The way in which uneven flow velocity enlarges the mixing zone can be understood in terms of a "mixing cell" model, as illustrated in Figure 2.2 .

As shown in Figure 2.2, Stalkup (1983) describes this as follows: "the individual Streamlines 1,2, and 3 follow a tortuous path through the porous medium, although the average direction of each streamline must be in the direction of mean flow. Suppose that different solvent concentrations initially are traveling along each streamline. The solvent concentrations associated with Streamlines 1 and 2 enter Pore A through small pore connections or pore throats. Within Pore A the solvent concentration is equalized by molecular diffusion such that a uniform concentration emerges from Pore A. The solvent of altered composition associated with Streamline 2 then mixes in Pore $\mathrm{C}$ with the solvent of composition associated with Streamline 3. In Pore C, diffusion again equalizes concentration such that solvent of twice-altered composition emerges from Pore C along Streamline 2. Mixing between Streamlines 1 and 2 then occurs in Pore D, etc."

\subsection{Generalized Models for Dispersion Phenomena}

Two generalized models for the dispersion phenomena have been developed from the continuum approach. They are the convection dispersion (or diffusion-type) model and the dispersion capacitance model.

\subsubsection{Convection Dispersion (or Diffusion-type) Model}

Taylor (1953) was the first to present a mathematical analysis of the transient convective diffusion equation to describe dispersion in fluid flow systems. Many other investigators including Aris (1956, 1957, 1959), Bear (1960, 1961), Brigham et al. (1961) and some others have also contributed to the development of this classic dispersion model. Most of the investigators approached the problem in a field of flow by first constructing the partial differential equation which describes the dispersion process and then solving it under the specified boundary condition. Scheidegger (1954) and Bear and Bachmat (1967) derived the general equation that describes the overall transport and mixing of fluids flowing through a porous medium as:

$$
\nabla(D \nabla C)-(\nabla \nabla C)=\phi \frac{\partial C}{\partial t}
$$

where:

$$
\begin{aligned}
& \nabla=\text { gradient operator } \\
& D=\text { dispersion coefficient }\left(\mathrm{cm}^{2} / \mathrm{sec}\right)
\end{aligned}
$$




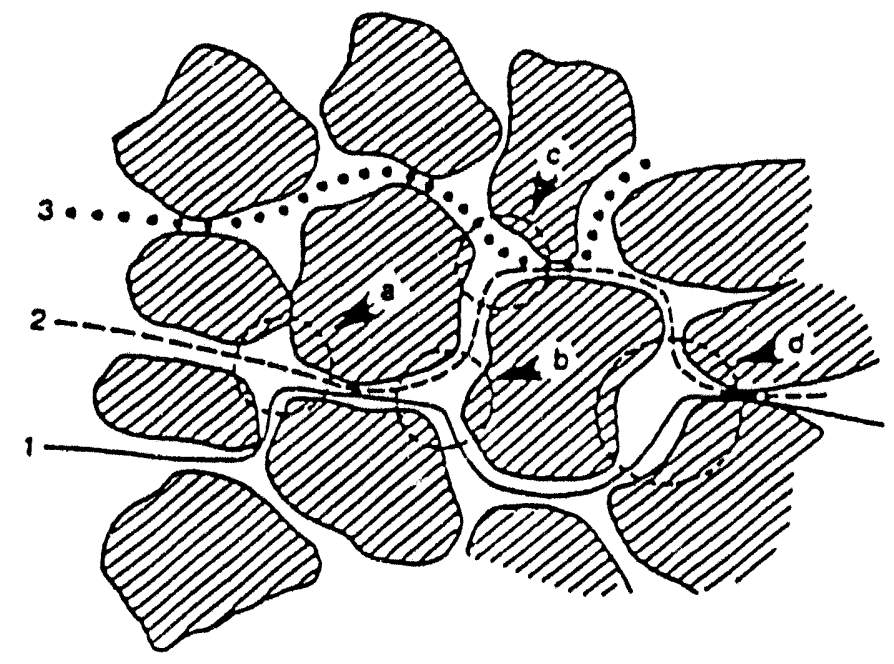

Figure 2.2: Microscopic convective dispersion (Stalkup, 1983) 
$\mathrm{C}=$ effluent concentration (mg/liter),

$\mathrm{V}=$ Darcy velocity $(\mathrm{cm} / \mathrm{sec})$,

$\phi=$ porosity of the porous medium (\% pore volume), and

$t=$ time (sec).

Since the dispersion process is influenced by many factors which are be discussed later, a "standard" system must be used to obtain consistent values of dispersivity for making correlations with other rock parameters. For such a purpose, the "standard" miscible displacement system is defined as follows:

(a) the porous medium has a constant cross section and length,

(b) the porous medium is initially 100 percent saturated with a single phase resident fluid (displaced fluid),

(c) the resident fluid is displaced by a displacing fluid having the same density and viscosity as the resident fluid,

(d) the compressibilities of the fluids and of the porous medium are negligible,

(e) the fluids flow at constant rates,

(f) the fluid flow rate is in the range of laminar flow,

(g) the dispersion coefficient is independent of the fluid concentrations,

(h) the effects of molecular diffusion and transverse dispersion are negligible compared with the longitudinal dispersion, and

(i) there is no chemical reaction taking place between the injected fluid and the porous medium or the resident fluid.

For such system, Eq.(1.1) is simplified as:

$$
D_{L} \frac{\partial^{2} C}{\partial x^{2}}-V_{\cdot} \frac{\partial C}{\partial x}=\frac{\partial C}{\partial t}
$$

where:

$D_{L}=$ the longitudinal dispersion coefficient $\left(\mathrm{cm}^{2} / \mathrm{sec}\right)$,

$\mathrm{x}=$ the distance along the system $(\mathrm{cm})$, and

$\mathrm{V}_{\mathrm{c}}=$ Dupuit-Forchheimer's interstitial velocity $(\mathrm{V} / \phi),(\mathrm{cm} / \mathrm{sec})$.

This equation has been solved with a set of initial and boundary conditions. Many 
different types of solutions have been obtained for different sets of boundary conditions. Kreft and Zuber (1978) compared these different types of boundary conditions and the resultant solutions. By introducing the concepts of the distinction between the concentration of solvent in resident fluid and in fluid flux, they concluded that the differences between the solutions for different injection-detection modes are essential in cases of low Peclet numbers (Peclet number is defined below). In systems having high Peclet numbers, the numerical differences between the solutions become negligible and the injection-detection mode is not so important.

By definition, Peclet number is a dimensionless ratio of the product of average interstitial velocity and particle diameter divided by the dispersion coefficient. It is defined as (Perkins and Johnston, 1963)

$$
P_{c}=\frac{d_{p} V_{c}}{D_{L}}
$$

where $P_{c}=$ Peclet number, and $d_{p}=$ particle diameter.

One solution of Eq. (2.1) has been developed with respect to the following initial and boundary conditions:

$$
\begin{array}{lll}
C=C_{0} & x=0 & t>0 \\
C=0 & x>0 & t=0 \\
C=0 & x \rightarrow \infty & t \geq 0
\end{array}
$$

The solution has been previously developed to yield the following (Sandrea and Nielsen, 1974):

$$
\frac{C}{C_{0}}=\frac{1}{2}\left[\operatorname{erfc}\left(\frac{x-v_{c} t}{\sqrt{4 D_{L} t}}\right)+\exp \left(\frac{v_{x} x}{D_{L}}\right) \operatorname{erfc}\left(\frac{x+v_{e} t}{\sqrt{4 D_{L} t}}\right)\right]
$$

Ogata and Banks (1961) have shown that the second term of Eq.(2.4) can be neglected when $D_{L} / v_{0} x$ is less than 0.0075 with an error of less than 5 percent. Thus, Eq. (2.4) can be expressed in a simpler form as:

$$
\frac{c}{c_{0}}=\frac{1}{2}\left[\operatorname{erfc}\left(\frac{x-v_{e} t}{\sqrt{4 D_{L} t}}\right)\right]
$$

The above equation can be used to determine the dispersion coefficient from a measured breakthrough curve. The equation indicates that the point where the relative 
concentration of a solute (or the displacing fluid concentration) is equal to 0.5 , occurs whenever $x=v_{0} t$ (i.e., whenever one pore volume of displacing fluid has been displaced). Also, it identifies the shape of the displacing concentration front (breakthrough curve) as being exactly symmetrical about the 0.5 relative concentration value (i.e., the solute distribution in porous media is Gaussian or normal).

Generally, one measures the effluent concentration at the outlet of the core in the laburatory. To compare the model equation with the experimental data, Brigham et al. (1961) proposed to set $x$ equal to $L$ (the length of medium) in Eq.(2.5) and calculate the concentration for various values of injected pore volume. For the "standard" miscible displacement system, the following relationships hold:

$$
\begin{aligned}
& x-V_{c} t=L\left(\frac{V_{p}-V_{t}}{V_{p}}\right) \\
& t=T_{b c}\left(\frac{V_{t}}{V_{p}}\right)=\frac{L}{V_{c}}\left(\frac{V_{t}}{V_{p}}\right) \\
& I=\frac{V_{t}}{V_{p}} \\
& \gamma=\frac{L V_{c}}{D_{L}}
\end{aligned}
$$

where:

$L=$ length of the system,

$V_{p}=$ pore volume of the porous medium,

$V_{1}=$ volume of injected displacing fluid,

$T_{b c}=$ breakthrough time of injected fluid at the outlet,

$I$ = pore volume number of injected fluid, and

$\gamma=$ dimensionless dispersion coefficient.

Substituting Eqs.(2.6) through (2.9) into Eq.(2.5) yields the solution to the convectiondispersion model as: 


$$
C=\frac{1}{2} e r f c\left[\frac{1-I}{\sqrt{\frac{4 I}{\gamma}}}\right]
$$

The plot of this equation gives an S-shaped curve as shown in Figure 2.1. Therefore, the laboratory measured S-shaped concentration profile can be matched with the convection-dispersion model by adjusting the dimensionless dispersion coefficient $(\gamma)$.

\subsubsection{Dispersion Capacitance Model}

The convection dispersion model has been widely used for solving dispersion problems in homogeneous materials. Laboratory experiments on sand packs usually resulted in almust symmetrical effluen: concentration profiles as predicted by Bq.(2.10). However, many tests carried out on actual reservoir consolidated rocks show a marked deviation from this ideal behavior. These concentration profiles as shown in Figure 2.3 were characterized by:

\# breakthrough of the 50 percent concentration prior to the injection of one pore volume of displacing fluid (Brigham et al, 1961), and

\# an asymmetrical effluent concentration profile showing a long tail on the last portion of the curve (Scheidegger and Larson, 1958).

Because of these deviations, most investigators have realized that the simple dispersion model (i.e., convection-dispersion model) can not effectiveiy describe the mixing process and a modified model must be developed. The most modified models which are frequently used in the dispersion studies are presented in the following sections.

\subsubsection{Brigham's Solution}

Brigham (1972) treated the problem in terms of the difference between in-situ concentration and flowing concentration. He noted that Bq.(2.2) and the boundary conditions presented in previous pages define the fluid concentration in-situ, while the laboratory measured fluid concentration at the outlet of the core is the average flowing concentration. Based on the material balance, Brigham derived the following relationship:

$$
C_{f}=C-\frac{D_{L}}{V_{l}}\left(\frac{\partial C}{\partial x}\right)
$$

where:

$$
C_{\mathbf{f}}=\text { fluid concentration measured in flux, }
$$




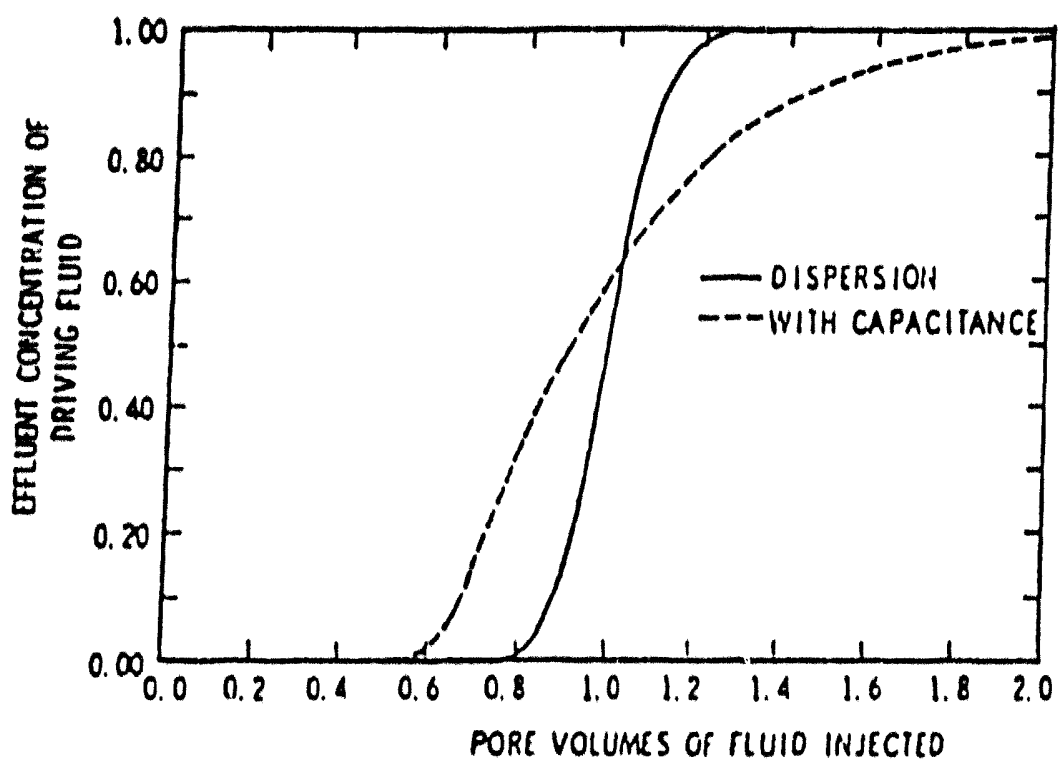

Figure 2.3: Concentration profiles showing capacitance effect (Brigham et al., 1961) 
$C=$ in-situ fluid concentration, and

$\partial C / \partial x=$ concentration gradient along the length of core sample.

About this equation, Brigham (1972) explained: "Since $\partial \mathrm{C} / \partial \mathrm{x}$ is negative, Bq.(2.11) says that the average concentration flowing across a plane is always greater than the concentration in the plane". At first this scoms paradoxical, but actually it is quite logical. This is somewhat analogous to the situation in two-phase flow in pipes. There, the in-situ gas/liquid ratio is nearly always different from the flowing gas/liquid ratio, because one fluid is flowing faster than the other. It is also analogous to BuckleyLeverett immiscible displacement, where the fraction flowing $\left(f_{m}\right)$ is different from the fraction in place $\left(s_{w}\right)$. In Bq. (2.11) it is evident that owing to the influence of the concentration gradient, the displacing fluid is flowing faster than the displaced fluid. Substituting Eq.(2.10) into Bq.(2.11) yields

$$
C_{f}=\frac{1}{2} \operatorname{erfc}\left(\frac{1-I}{\sqrt{\frac{4 I}{\gamma}}}\right)+\frac{1}{\sqrt{4 \pi \gamma I}} e^{-\left(\frac{1-1}{\sqrt{\frac{\mu}{\gamma}-}}\right)^{\prime}}
$$

Further by setting $I=1.0$, the above equation gives the effluent concentration at one pore volume of injection:

$$
C_{f, 0 / 01}=\frac{1}{2}\left[1+\frac{1}{\sqrt{\pi y}}\right]
$$

Apparently the effluer ' concentration at one pore volume injection is always greater than 50 percent. In this case, the material balance is preserved in Figure 2.3 where the areas to the right and the left of 1 pore volume are equal. So, Brigham (1972) suggested that Bq.(2.12), rather than Bq. (2.10), should be applied to match laboratory measured data.

Although Eq.(2.12) can match the front part of a concentration profile, it can not match the tail part of the concentration profile because the second term on the right side of this equation is relatively too small compared with the first term. Especially for the concentration profiles with very long tails, the convection-dispersion model fails to match. For these cases, the next section presents a more complicated model which should be used.

\subsubsection{Coats and Smith's Solution}

Tumer (1958) first developed a model of flow systems in which there are stagnant 
pockets of various depths opening on the flow channels. Carberry and Bretton (1958) called these "stagnant pockets" the "dead-end pores". The effect of these dead-end pores were then referred to as the "capacitance". Aris (1959) applied Turner's "stagnant pocket" model to derive a general method for calculating Taylor's dispersion coefficient.

The concept of capacitance implies the presence of a nonflowing volume in the medium. If it is to have an effect on the flowing stream, this stagnant volume must be connected to the flowing volume by some sort of resistance to material transport. Hence, Deans (1963) introduced two new parameters: stagnant volume fraction (1-f) and a masstransfer factor (M). As a result, Deans developed a three-parameter mathematical model to predict the longitudinal dispersion process. Later, based on Deans' work, Coats and Smith (196.4) fully developed the capacitance-dispersion model to take into account the effect of a stagnant pore volume on the growth of the mixing zone. The modified equations are:

$$
\begin{aligned}
& D_{L} \frac{\partial^{2} C}{\partial x^{2}}-V_{\bullet} \frac{\partial C}{\partial x}=f \frac{\partial C}{\partial t}+(1-f) \frac{\partial C^{\bullet}}{\partial t} \\
& (1-f) \frac{\partial C^{\bullet}}{\partial t}=M\left(C-C^{\bullet}\right)
\end{aligned}
$$

where:

$$
\begin{aligned}
& C^{*}=\text { in-situ concentra'ion of fluid in stagnant pockets, } \\
& \mathbf{f}=\text { flow fraction of the pore volume, and } \\
& M=\text { mass transfer coefficient between flowing fluid and stagnant fluid. }
\end{aligned}
$$

For sufficiently small velocity, the mass transfer process is essentially instantaneous. The model reduces to the convection-dispersion model, Eq.(2.2). For sufficiently large velocity, the mass transfer is negligibly small and the model also reduces to Eq.(2.2). From this fact, it can be established that the dispersion capacitance model is a more general model than the convection dispersion model.

The solution to Eqs. (2.14) and (2.15) has been given by Coats and Smith (1964) and later modified by Brigham (1972). The solution is complex and is usually expressed in terms of an integral function as:

$$
C(J)=\frac{2 e^{J}}{\pi} \int_{0}^{\infty} \frac{e^{\frac{\gamma}{2}\left(1-J \pi \cos \frac{1}{2}\right)}}{a_{1}^{2}+a_{2}^{2}}\left[a_{1} \cos (\beta J-w)+a_{2} \sin (\beta J-w)\right] d \beta
$$

where $J=I / f$, with other terms as defined in the Nomenclature. 
Further, by considering the difference between the in-situ concentration and the concentration in flux as indicated by Bq.(2.11), Eq.(2.16) should be modified accordingly as follows:

$$
C_{\gamma}(J)+\frac{2 e^{J}}{\pi} \int_{0}^{\infty} \frac{e^{\frac{\gamma}{2}\left(1-J \cos \frac{0}{2}\right)}}{a_{1}^{2}+a_{2}^{2}}\left[\Gamma_{1} \cos (\beta J-w)+\Gamma_{2} \sin (\beta J-w)\right] d \beta
$$

where

$$
\begin{aligned}
& \Gamma_{2}=\frac{a_{2}}{1}\left[1+\sqrt{\rho} \cos \left(\frac{\theta}{2}\right)\right] \frac{a_{1} w}{\gamma} \\
& \Gamma_{1}=\frac{a_{1}}{1}\left(1+\sqrt{\rho} \cos \left(\frac{\theta}{2}\right)\right] \frac{a_{2} w}{\gamma}
\end{aligned}
$$

Obviously Bq. (2.17), rather than Bq.(2.16), should be used for matching laboratory data.

In addition, it is noted that there are throe adjustable parameters in this model. They are flow fraction $(f)$, the mass transfer coefficient $(M)$, and the dispersion coefficient $\left(D_{\mathcal{L}}\right)$. Their different effects on the solution equation are illustrated in Figure 2.4. Because of this flexibility, it is not surprising that Eq.(2.17) should be better able to match any type of laboratory measured concentration profiles. However, Coats and Smith (1964) wamed that a better match is no proof that capacitance is an actual physical phenomenon, rather than merely a conceptual one.

\subsection{Techniques for Determining Dispersivities}

Currently, dispersivity can not be measured directly. It is calculated from the measured S-shaped concentration profile. Concentration profiles can be obtained either in the laboratory by conducting miscible displacement tests on core samples or in the field by tracer tests. In practice, three general techniques are usually employed to obtain rock dispersivities from the measured S-shaped concentration profiles. They are: (1) graphical method, (2) analytical method, and (3) numerical or finite difference method. Poulin (1985) compared these methods and found that the graphical method is more sensitive to flow rate variation than the other two methods. At sufficient 'ow flow rates, all three methods provided equivalent results. However, since the graphical method is sasier to use than the other two methods, it is discussed briefly below.

Basically, the graphical method is based on the analytical solution to the convection dispersion model It is known that Eq. (2.10) defines the well known S-shaped curve of the error function integral. A plot of this equation will yield a straight line on arithmetic 

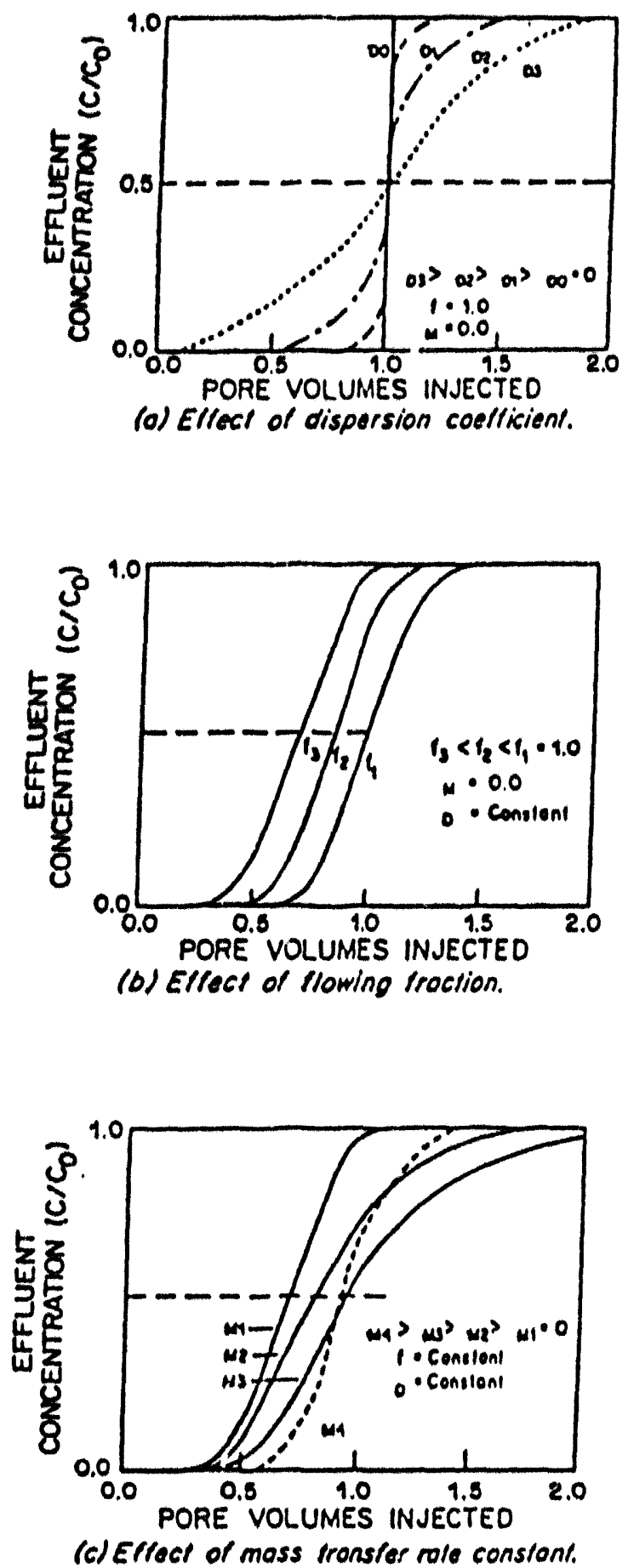

Figure 2.4: Effects of the parameters in capacitance model on the shapes of concentration profiles (Batycky et al., 1982) 
probability co-ordinate paper. However, Bq.(2.10) defines the concentration profile in the porous medium at a given time, while the experimental cata gives the concentration at a given point as the constantly growing mixed zone moves past the observer. Therefore, a volume modifying function is defined by Brigham (1972) as follows:

$$
\lambda=\frac{\frac{V_{1}}{V_{p}}-1}{\sqrt{\frac{V_{1}}{V_{p}}}}=\frac{I-1}{\sqrt{I}}
$$

where $\lambda$ is the volume modifying function parameter. With this definition, Bq.(2.10) becomes

$$
C=\frac{1}{2}\left[1+\operatorname{erf}\left(\sqrt{\frac{\gamma}{4} \lambda}\right)\right]
$$

It is necessary to define the matationship between the error function parameter $\lambda$ and the rate of growth of the mixed zone which is characterized by the dispersion coefficient $D_{L}$. Taylor (1953) has shown that $D_{L}$ can be related to the length of the transition zone between any two specified compositions. Because of the asymptotic behavior of the complementary error function, it is not practical to define the mixing zone as that in which $\mathrm{C}$ ranges from zero to unity. Instead, it is customary to select arbitrary values of concentration, such as 10 percent and 90 percent, to define the mixing zone boundaries. Substituting these values for $\mathrm{C}$ in Bq.(2.21) yields

$$
\lambda_{90}-\lambda_{10}=\frac{3.625}{\sqrt{\gamma}}
$$

where:

$\lambda_{90}=$ the $\lambda$ value corresponding to the 90 percent concentration, and

$\lambda_{10}=$ the $\lambda$ value corresponding to the 10 percent concentration, and Obviously, $\left(\lambda_{90}-\lambda_{10}\right) / 3.625$ is the slope of the straight line on an arithmetic probability coordinate paper. Then combining Eq.(2.22) with Eq.(2.9) yields the equation for calculating the dispersivity:

$$
\alpha_{D}=L\left(\frac{\lambda_{90}-\lambda_{10}}{3.625}\right)^{2}
$$

where $\alpha_{D}$ is a rock dispersivity (cm). 
Similarly, if 20 percent and 80 percent of concentration are defined as the mixed zone boundaries, the equivalent equation is

$$
\alpha_{D}=L\left[\frac{\lambda_{30}-\lambda_{20}}{2.38}\right]^{2}
$$

How to determine dispersivity of a porous medium using the graphical method is illustrated in Figure 2.5.

\subsection{Controlling Factors for Dispersivity}

Many factors influencing dispersivity values have been recognized. These factors include (1) ratio of particle diameter to sample medium diameter, (2) particle size distribution (3) particle shape, (4) packing or permeability heterogeneities, (5) difference of viscosities, (6) difference of densities, (7)non-laminar (non-Darcy's) flow, (8) an immobile phase, (9) wettability, and (10) diffusivity. The effects of these variables are discussed in detail by Perkins and Johnston (1964), Raimondi et al. $(1959,1961)$, Orlob and Radhakrishna (1958), Blackwell (1962), Stalkup (1970, 1983), Kasraie (1979), Maini et al. (1986), Wong (1988), and others. Most of the experimental and analytical results have shown the dependence of the magnitude of the dispersion coefficient on the fluid velocity and the effective grain size of the porous medium. This dependency comes about because, provided the sand is uniform, the geometric characteristics are described by a single parameter --- the sand-grain diameter (Ogata, 1970). Based on the experimental data, Blackwell (1962) established a graph (Figure 2.6) which shows the relationship between the magnitude of the dispersion coefficient and grain sizes. Scheidegger (1957) has shown that there are two possible extremes for the form of dispersion coefficient: one directly proportional to velocity, and the other proportional to the square of velocity, i.e.,

$$
D_{L} \sim \sim \alpha_{D, s} V_{e}
$$

and

$$
D_{L} \sim \sim \alpha_{D, d} V_{e}^{2}
$$

where:

$\alpha_{D, g}=$ the geometric dispersivity applicable where there is no appreciable molatar transverse diffusion, and

$\alpha_{D, d}=$ the dynarnic dispersivity derived by a dynamic procedure where there is appreciable mixing by molecular transverse diffusion.

In other words, the first equation reflects the situation where there is enough time for complete mixing of the fluids while the second equation represents incomplete mixing. 


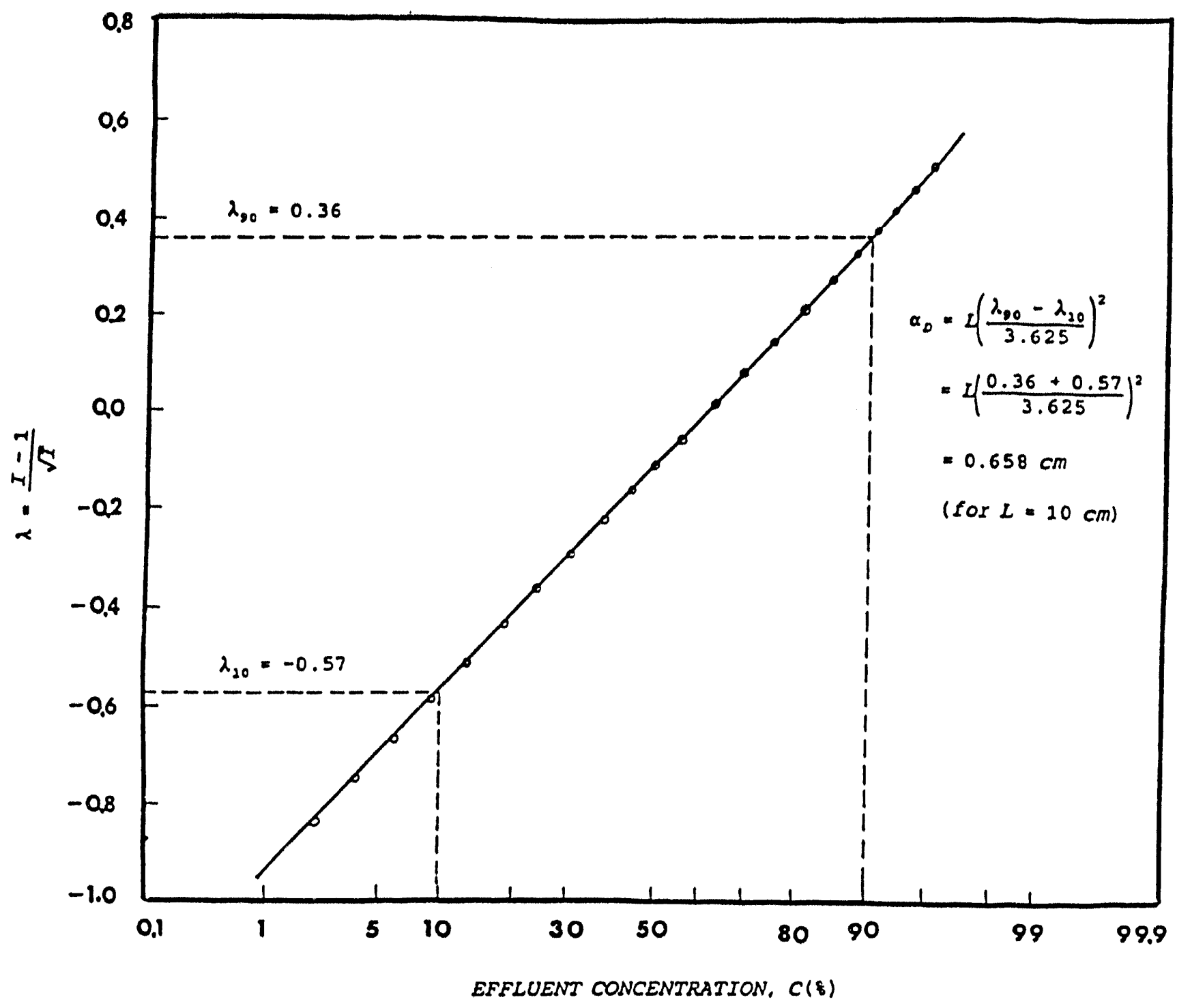

Figure 2.5: Illustration of the graphical method to determine dispersivity 


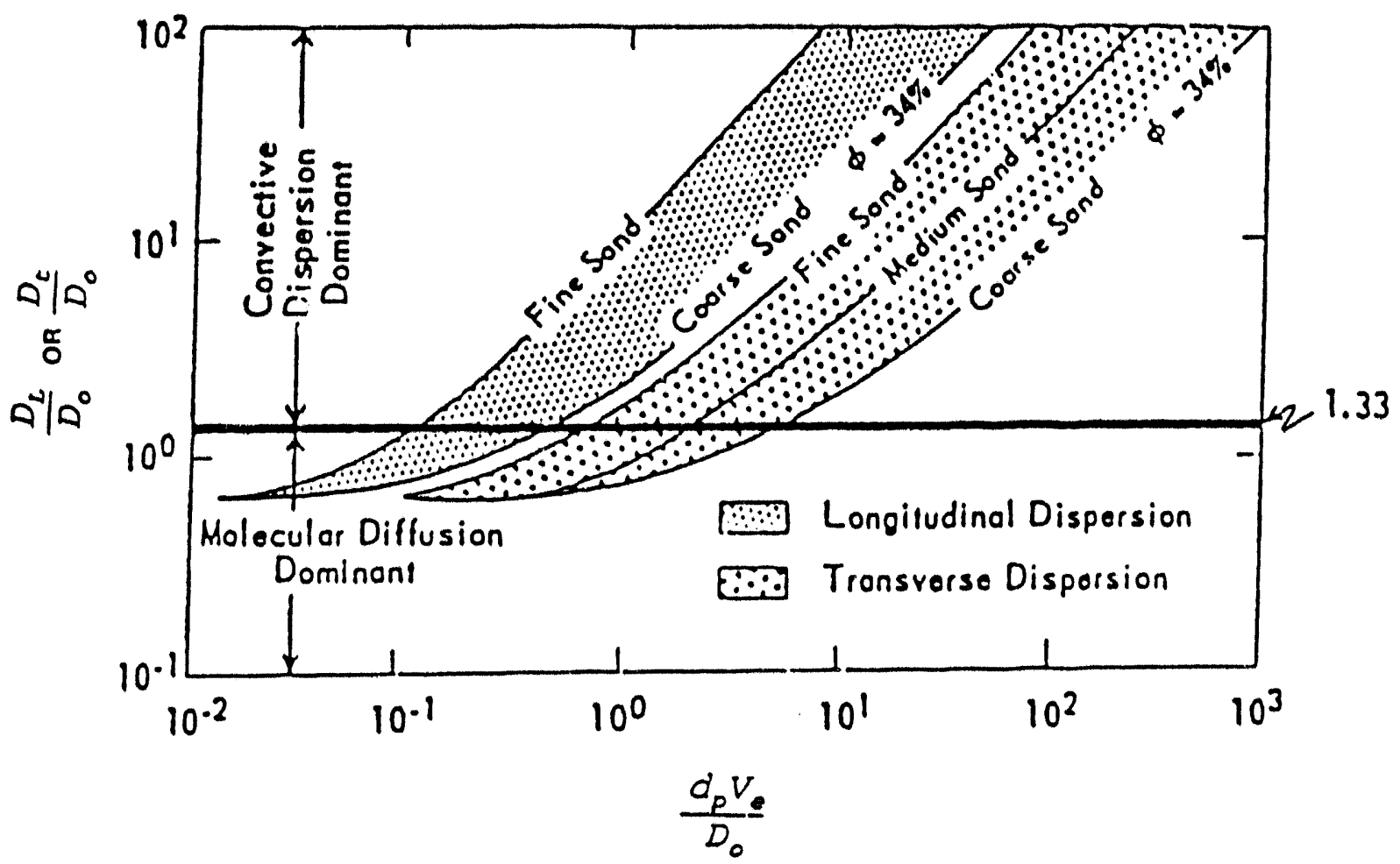

Figure 2.6: Dispersion coefficient versus peclet number and grain size (Blackwell, 1962) 
Generally, this relationship is expressed as

$$
D_{L} \sim \alpha_{D} V_{e}^{n_{*}}
$$

where $n_{v}$ is the velocity exponent which ranges from 1 to 2 .

Nearly all literature data shows that this velocity exponent, $\mathrm{n}_{v}$, is slightly greater than 1.0 and less than 1.2. Blackwell et al. (1958) found $\mathrm{n}_{v}=1.24,1.20$, and 1.19 in the 0.47 $\mathrm{mm}$ beads, $0.100 \mathrm{~mm}$ beads, and the Berea core, respectively. Pleshek (1968) observed a significant effect of particle size or permeability on this exponent. But another possible factor - wettability, which has been neglected before, might also influence this exponent as well. In general, experiments seem to indicate that Bq.(2.25) corresponds to physical reality (Scheidegger, 1961). The present research will use the first relationship, Eq.(2.25) for defining dispersivity. For the sake of simplicity, the parameter $\alpha_{D_{s}}$ will be simply written as $\alpha_{D}$.

Raimondi et al. (1959) have given a general form of the relationship between the dispersion coefficient, grain size, and velocity:

$$
D_{L}=D_{m}+c \sigma_{H} V_{c} d_{p}
$$

where:

$$
\begin{aligned}
& D_{m}=\text { effective molecular diffusion coefficient }\left(=D_{d} / \tau^{2}\right), \\
& \tau=\text { tortuosity, } \\
& D_{0}=\text { fluid diffusivity, } \\
& c=\text { a constant, and } \\
& \sigma_{H}=\text { a parameter that is a function of heterogeneity factor }(H) .
\end{aligned}
$$

Note that $\sigma_{H}$ is called the heterogeneity-related parameter because it is different from the heterogeneity factor defined by Koval (1963).

Perkins and Johnston (1963) have shown that the longitudinal dispersion coefficient for unconsolidated sand of bead packs can be represented by the following equation:

$$
D_{L}=D_{m}+1.75 V d_{p} \quad\left[2<\frac{V d_{p}}{D_{0}}<50\right)
$$

Furthermore, Aris and Amundson (1957), Carberry (1958), and Prausnitz (1958) have shown that $c=0.5$ by using the mixing-cell theory. This would then give a value of about $\sigma_{\mathrm{H}}=3.5$ for the typical random pack. However, we should expect that $\sigma_{\mathrm{H}}$ would be much larger than 3.5 for consolidated sandstones. 


\subsection{Correlations of Dispersivity with Rock Petrophysical Properties}

A considerable amount of research effort has been devoted to the development of a quantitative relationship describing the dispersivity as a function of various displacement parameters. The most sought relationship is that between dispersivity and permeability because both parameters are directly related to fluid flow.

\subsubsection{Dlspersivity versus Permeabllity}

Harleman et al. (1963) have derived the semi-theoretical correlations between the dispersion coefficient, permeability, and the average particle size. In these correlations, Harleman and his co-workers used the particle size Reynolds number and permeability Reynolds number as follows:

$$
\frac{D_{L}}{\mu}=\psi\left(\frac{V d_{s 0}}{\mu}\right)^{n_{\nu}}=\psi R_{d_{\mu}}^{n_{m}}
$$

and

$$
\frac{D_{L}}{\mu}=\chi\left(\frac{V_{c} \sqrt{K}}{\mu}\right)^{n_{t}}=\chi R_{k}^{n_{t}}
$$

where:

$$
\begin{aligned}
& \mu=\text { fluid kinematic viscosity, } \\
& \psi, \chi=\text { dimensionless coefficients presumably dependent on only the particle shape } \\
& \text { and pore size distribution, } \\
& \mathrm{n}_{\mathrm{t}}=\text { an exponent which is a function of primarily the size distribution, } \\
& R_{d s 0}=\text { particle size Reynolds number }\left(=V_{\sigma} K^{0.5} / \mu\right) \text {, } \\
& \mathbf{K}=\text { permeability, } \\
& d_{30}=50 \text { percent particle size, and } \\
& \mathbf{R}_{\mathbf{t}}=\text { permeability Reynolds number }\left(=\mathrm{V}_{\mathrm{\sigma}} \mathrm{K}^{0.5} / \mu\right) \text {. }
\end{aligned}
$$

For the media composed of spheres, $\psi=0.66$ and $x=54$. For the angular particles, $\psi=0.90$ and $x=83$. The exponent $n_{k}$ was found to be independent of particle size and shape, being equal to 1.2 for all cases (see Figures 2.7 and 2.8). Preliminary observations indicate that $n_{4}$ decreases with increasing nonuniformity of the particle sizes of a medium and approaches unity for highly nonuniform media.

Further, based on the data provided by Harleman et al., rock dispersivities were calculated and then plotted against permeabilities. As shown in Figure 2.9, a general linear relationship between dispersivity and permeability can be observed. 84 percent 


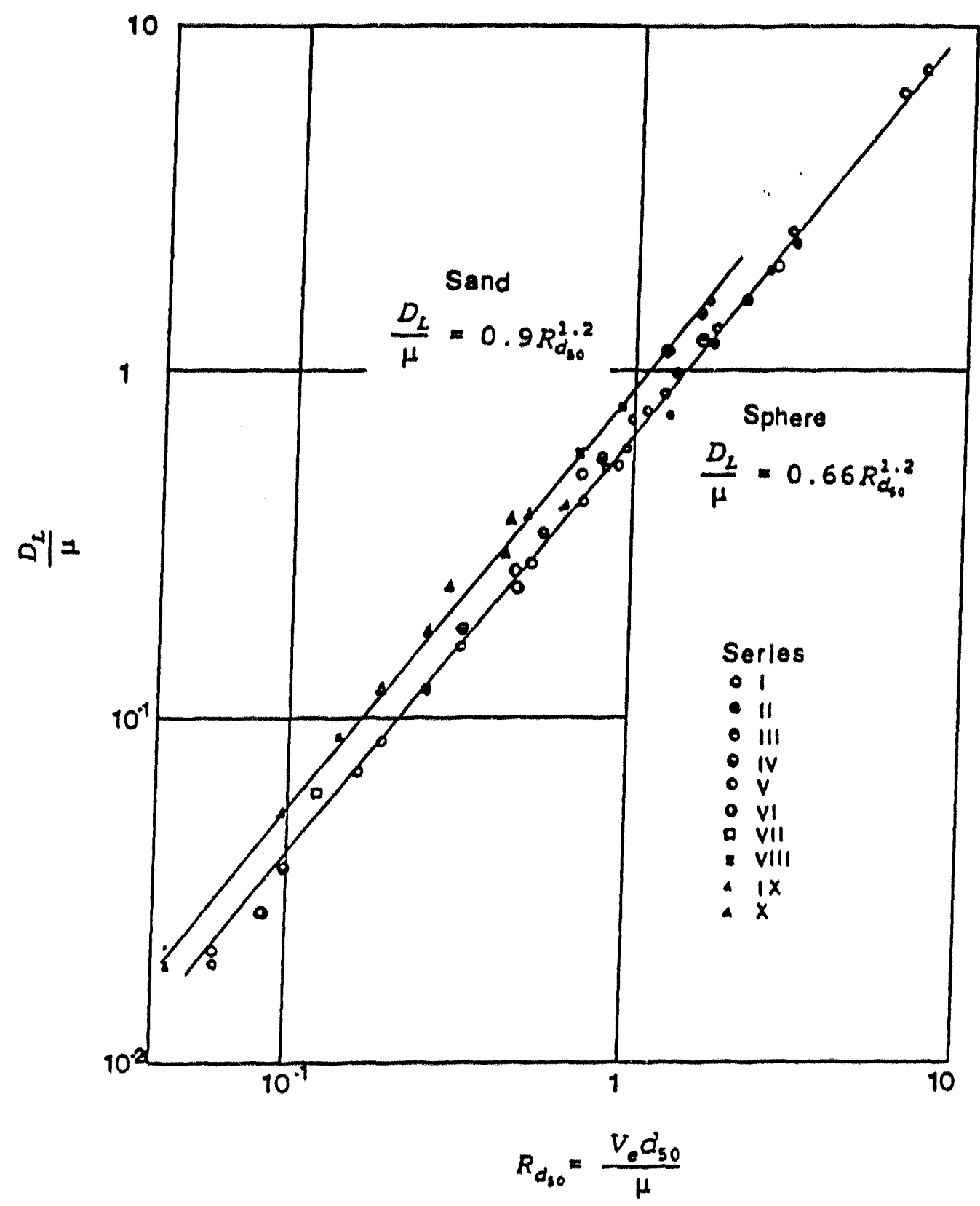

Figure 2.7: Correlation between dispersion and particle size (Harleman et al., 1963) 


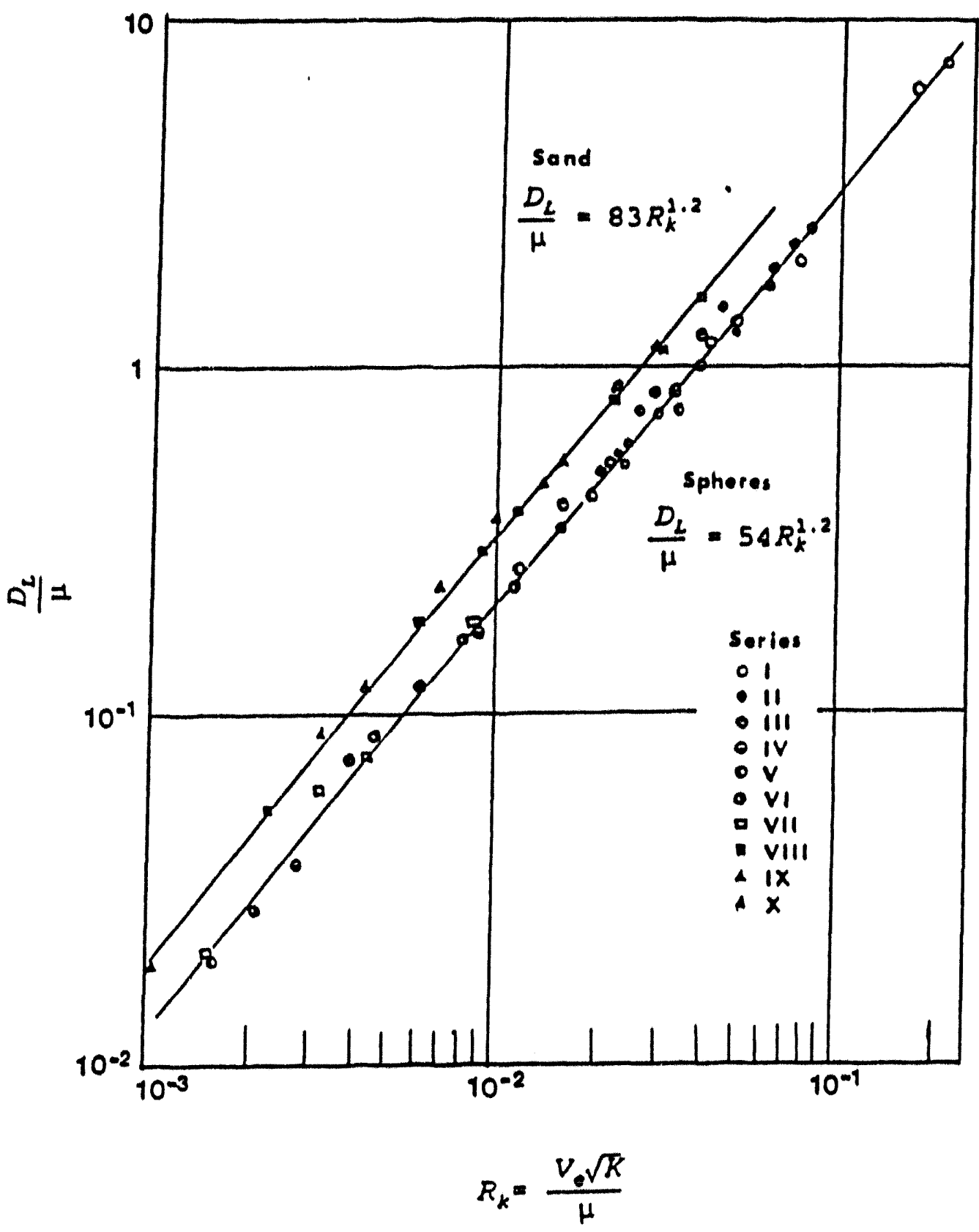

Figure 2.8: Correlation between dispersion and permeability (Harleman et al., 1963) 


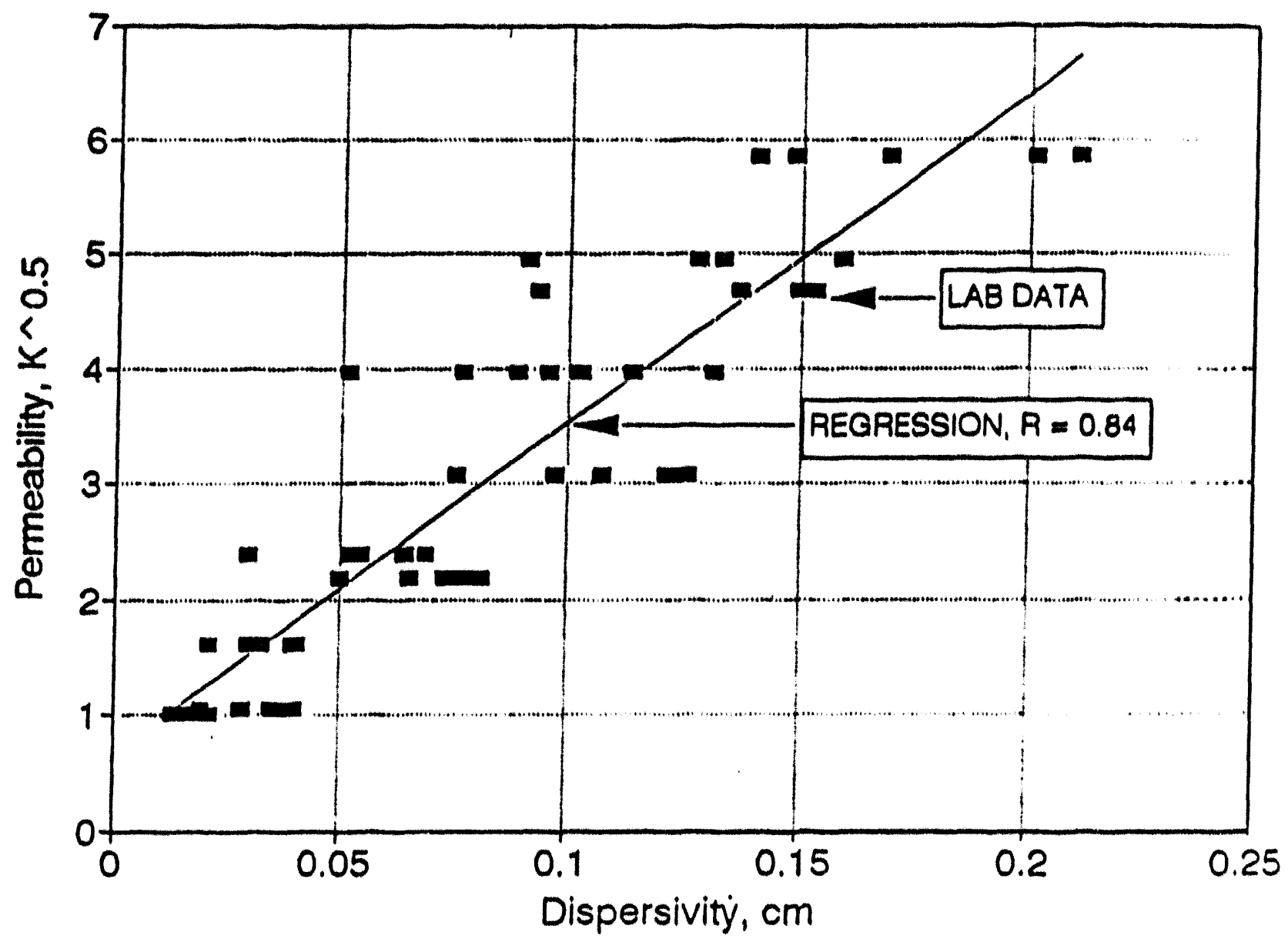

Figure 2.9: Correlation between dispersivity and permeability (data from Harleman et al., 1963) 
of the variance in the data can be described by this correlation.

The variation of the data in Figure 2.9 could be influenced by many factors such as different sample lengths and heterogeneities. Similar correlations have been made by Pleshek (1968) and Niemann (1969) through experiments on nonuniform heterogeneous porous media. More recent atudies by Chen of al. (1984), Patel and Greaves (1987), and Menzie of al. (1989) also indicate the dependence of dispersivity on the media permeability.

\subsubsection{Dispersivity Versus Other Rock Parameters}

Dispersivity has also been correlated with other rock parameters. Excellent work has been done by Klotz and Moser (197a). They performed about 2500 dispersion tests on both artificial and natural materials to determine the dependence of the longitudinal dispersivity on the parameters of the streaming fluid and on the characteristics of the porous media. These artificial and natural materials are different in grain size, grain shape, grain roughness and angularity, compactness, and by verious uniformity coefficients of grain-size distribution. Table 2.1 lists the qualitative relationships between dispersivity and those rock parameters. For comparison between dispersivity and permeability, the relationships between rock permeability and those rock parameters are also listed in the table 2.1. It can be scen from this comparison that although a unique relationship may be expected between dispersivity and permeability, it is difficult to establish such a relationship for natural reservoir rocks. This is because, with the expectation of the grain size, the dependence of all other rock parameters on dispersivity and permeability is inversely related. The dispersivity must therefore be an independent parameter in studies of petrophysics (Klotz and Moser, 1974).

\subsection{Scale Dependency of Dispersivity}

Laboratory experiments in granular materials have yielded values of longitudinal dispersivity which are typically in the range of $0.001 \mathrm{~cm}$ to $6 \mathrm{~cm}$, while the dispersivity values determined from field experiments showed three to four orders of magnitude larger than that determined from laboratory experiments (Anderson, 1979; Freeze and Cherry, 1979).

\subsubsection{Arguments on Scale Dependency}

From the literature, two opposite arguments can be found about the scale dependency of dispersivity values:

(1) Most investigators (Pickens and Grisak, 1981a \& 1981b; Moltz et al., 1983; Davis, 1986; and Taylor et al., 1987) believe that dispersivity should be scale dependent in the field scale, but should be independent in the laboratory scale, because laboratory 
Table 2.1: Corrolation of Dispersivity with Rock Panameters (after Klotz and Moser, 1974)

(f increasing, and + decrasing)

\begin{tabular}{|c|c|c|}
\hline Parameters & $\begin{array}{c}\text { Permeability } \\
k \text { (md) }\end{array}$ & $\begin{array}{c}\text { Dispersivity } \\
\alpha_{D}(\mathrm{~cm})\end{array}$ \\
\hline $\begin{array}{l}\text { Kinematic viscosity of } \\
\text { solvent solution, } \mu\end{array}$ & $\mu \uparrow: x+$ & $\mu \uparrow: \alpha_{D} \uparrow$ \\
\hline Temperature, $T_{1}$ & $T \uparrow: K \uparrow$ & $T \uparrow: \alpha_{D} \downarrow$ \\
\hline Porosity, $\phi$ & $\phi \uparrow: K \uparrow$ & $\phi \uparrow: a_{D} \downarrow$ \\
\hline Effective grain size, $d_{p}$ & $a_{p} i: k \uparrow$ & $a_{p} \uparrow: \alpha_{0} \uparrow$ \\
\hline $\begin{array}{l}\text { Deviation of grain shape } \\
\text { from a sphere, sh }\end{array}$ & $\operatorname{sh} \uparrow: k+$ & $\operatorname{sh} \uparrow: a_{D} \uparrow$ \\
\hline $\begin{array}{l}\text { Roughness and angularity } \\
\text { of grains, G }\end{array}$ & G $1: k l$ & $G 1: \alpha_{D} 1$ \\
\hline $\begin{array}{l}\text { Uniformity coeficient, } \\
u_{c}\end{array}$ & $U_{c} \uparrow: K \downarrow$ & $u_{C} \uparrow: \alpha_{D} \uparrow$ \\
\hline
\end{tabular}


experiments are typically conducted in relatively homogeneous media and therefore, laboratory dispersion measurement can not reflect the reservoir heterogeneity which is reflected by the field test (Kelkar and Gupta, 1988).

A counterexample is illustrated in Figure 2.10. In this figure, three groups of dispersion measurements conducted on three sand packs fall nearly in a straight line. These three and packs were the same except for the length. Respectively, they are $83.3,167.2$, and $678 \mathrm{~cm}$ long. From this graph and Bq. (2.24), the dispersivities of the throe sand packs were calculated to be $0.92,1.84$, and $7.46 \mathrm{~cm}$, respectively.

Since the sand pa ks are the same except for their lengths, their dispersivities are expected to be equal. However, the calculated dispersivity values clearly indicated strong dependency on length scale. This plot was used by Brigham at al. (1961) to illustrate the graphical method for determining the dispersion coefficient. They did not acknowledge this scale-dependency of the measured dispersivities in their paper.

(2) Some investigators (Yellig and Baker, 1981) believe that dispersivity should be scale dependent in the haboratory scale, but should be scale independent in the field scale. This point of view is mainly possessed by Yellig and Baker (1981). Through examining the Coats-Smith capacitance model, Yellig and Baker defined the separation distance between $C$ and $C^{\prime}$ 'flood fronts as:

$$
d_{d}=\frac{L(1-f) V_{c}}{M L+(1-f) V_{0}}
$$

where $d_{1}$ is the separation distance between the flood fronts of concentration $C$ and $C^{*}$. This equation indicates that as $L$ increases, $d_{1}$ approaches a stabilized value. By assuming $d$, to be 98 percent of the stabilized distance, Yellig and Baker derived the critical system length as:

$$
L_{c}=49 \frac{(1-f) V_{c}}{M}
$$

where $L_{0}$ is the critical system length. If the travel distance of the solvent is larger than this critical length, the measured dispersivity should be scale independent; and if the travel distance is less than this critical length, dispersivity should be scale dependent. Since almost all system lengths in the laboratory scale are less than this critical length, the measured dispersivity should be scalo-dependent.

A counterexample of this viow is illustrated in Figure 2.11. It is obvious that the measured dispersivities increase as the travel distance of the tracer increases. It was surprising that even through the travel distance was up to 10,000 meters, the measured dispersivities still did not stabilize as predicted by Eq.(2.32). 


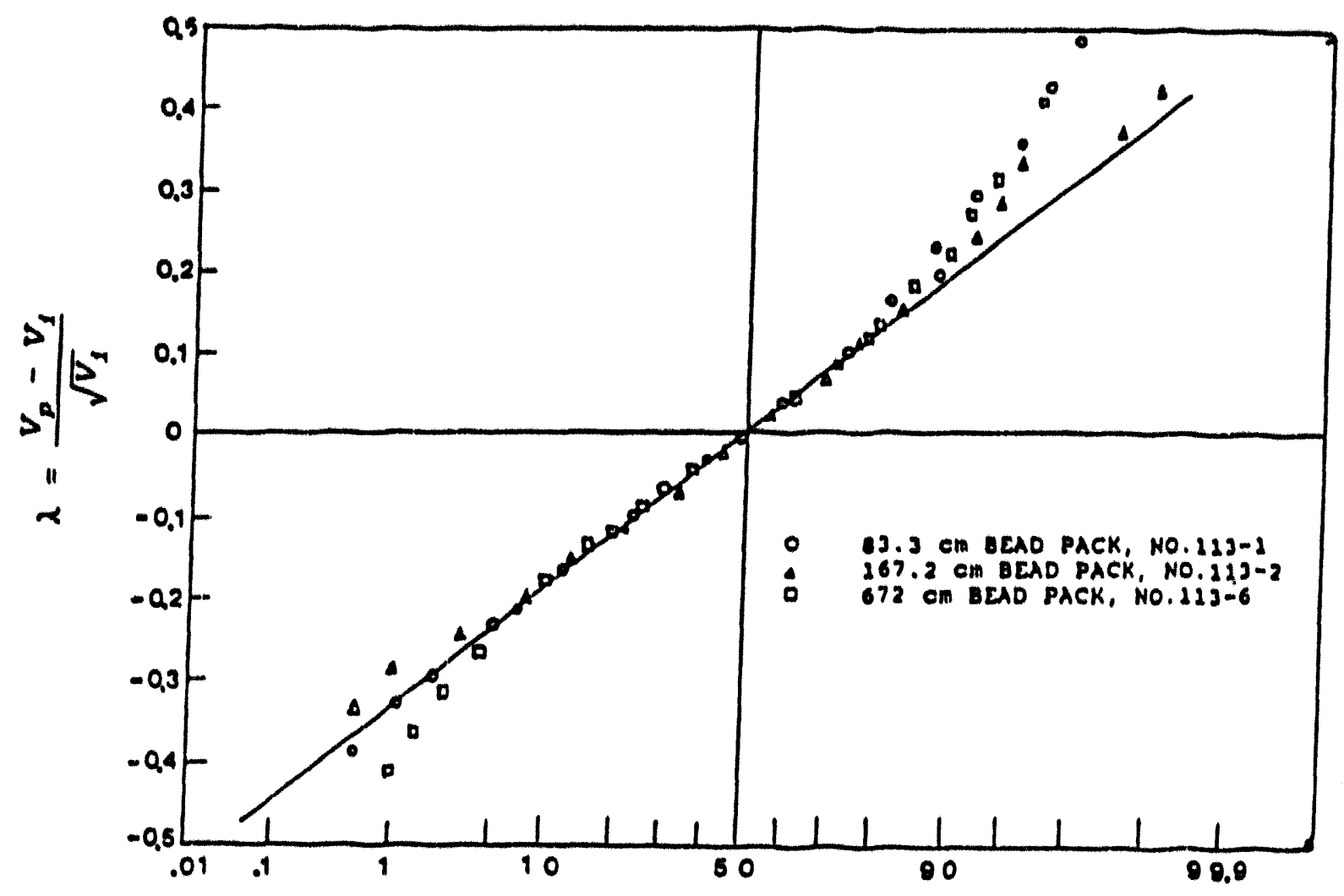

- DISPLACING FLUID

Figure 2.10: Laboratory measured dispersivities on sand packs showing scale dependency (Brigham et al., 1961) 


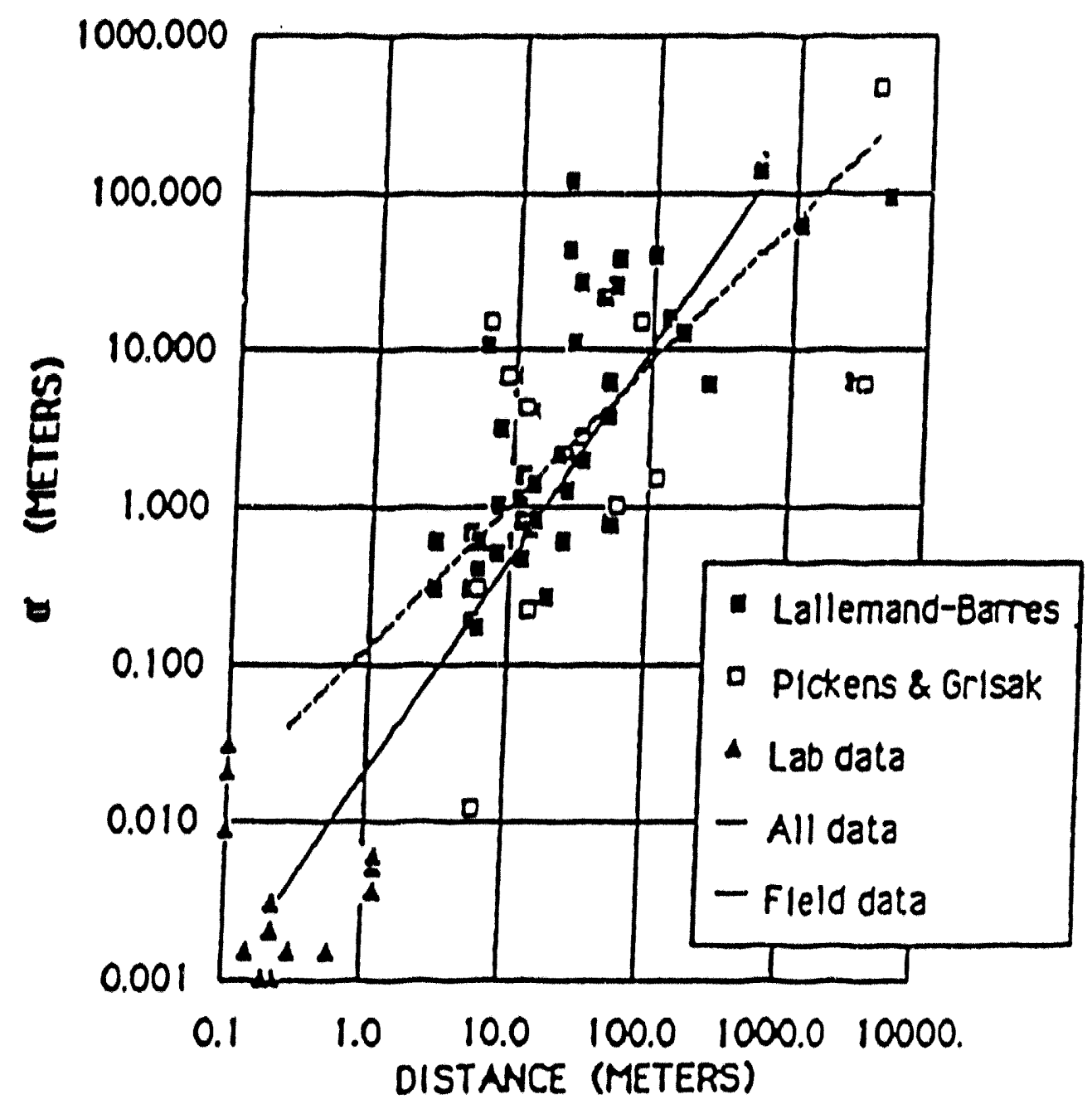

Figure 2.11: Field and laboratory dispersivities showing scaledependency (Lake et al., 1986) 
Using the data presented in Figure 2.11, Lake et al. (1986) obtained the following correlations:

$$
\alpha_{D}=0.229 L^{0.73 s}
$$

for field data only, and

$$
\alpha_{D}=0.044 L^{1.13}
$$

for all data.

A rather simple relationship used by Pickens and Grisak (1981a) in simulating solute transport in porous media is:

$$
\alpha_{D}=0.1 L
$$

Through the above discussion, it seems that both laboratory and field measured dispersivities are scale dependent.

\subsubsection{Hypotheses for Scale Dependency}

Many hypotheses to explain the scale effects have been proposed by investigators. Theis (1962) concluded that the increased values of hydrodynamic dispersion observed in the field, compared to the lab, must result from wide distribution and heterogeneities in hydraulic conductivities and velocities within the reservoir which may not bo accurately represented in laboratory core samples. Schwartz (1977) numerically simulated a hypothetical regional system and found that it was impossible to define a unique dispersivity value unless the hydraulic conductivity contrasts were homogeneously distributed within the reservoir. Pickens et al. (1978) observed that the size of the sample volume has a measurable influence on calculated dispersivity values. Domenico and Robbins (1984) postulated that another, perhaps more common, reason is the inability of the fitted model to account for the three-dimensional nature of dispersion in field conditions. Dominco and Robbins (1984) also indicated that the wide variation in field values may also be due to the sampling techniques employed. 


\section{CHAPTER 3}

\section{THEORETICAL DEVELOPMENT OF THE RELATIONSHIP BETWEEN DISPERSIVITY AND LOG-DERIVABLE ROCK PROPERTIES}

As discussed in the previous chapter, dispersivity is a characteristic of a porous medium. If it can be determined from well logs, a new dimension will be added to the characterization of reservoir rocks.

To determine dispersivities from well logs, a relationship between dispersivity and the log-derivable parameters must first be established. Since all macroscopic rock properties are influenced, to a greater or lesser degree, by the pore structure, a pore structure parameter will be crucial to the development of the relationships between rock property parameters. As a good connecting parameter between rock properties, tortuosity is chosen as the pore structure parameter to bridge the gap between dispersivity and the log-derivable rock parameters.

During the past several decades, tortuosity has been widely used in fluid flow studies and some other areas, but unfortunately, it little research were completed on its effect on rock properties. To date, great confusion still exists in the literature. This confusion will be discussed in this chapter.

\subsection{The Concepts of Tortuosity and the Tortuosity Factor}

When a fluid or an electrical current flows through a porous medium, fluid particles or ions do not travel along a straight path as in a pipe; instead, they follow a tortuous path inside the medium as shown in Figure 3.1. To describe this tortuous path, Carman (1937) defined a parameter, called tortuosity, as the ratio of the actual length of path followed by a particle or ion in passing through the porous medium, $L_{c}$, to the geometrical length of the medium, $\mathbf{L}$. Mathematically, it is expressed as:

$$
\tau=\frac{L_{e}}{L}
$$

However, even though the tortuosity of a rock is determined primarily by the manner in which the pore channels twist and turn in going around sand grains, there is also an effect due to the variation of the cross-sectional area of the tortuous paths. This can be represented by Figure 3.2. In this figure it is apparent that a small part of fluid or current will flow along a perfectly straight streamline, and a larger part will flow along curved streamlines. Therefore, the average length of flow path from plane $x$ to plane 


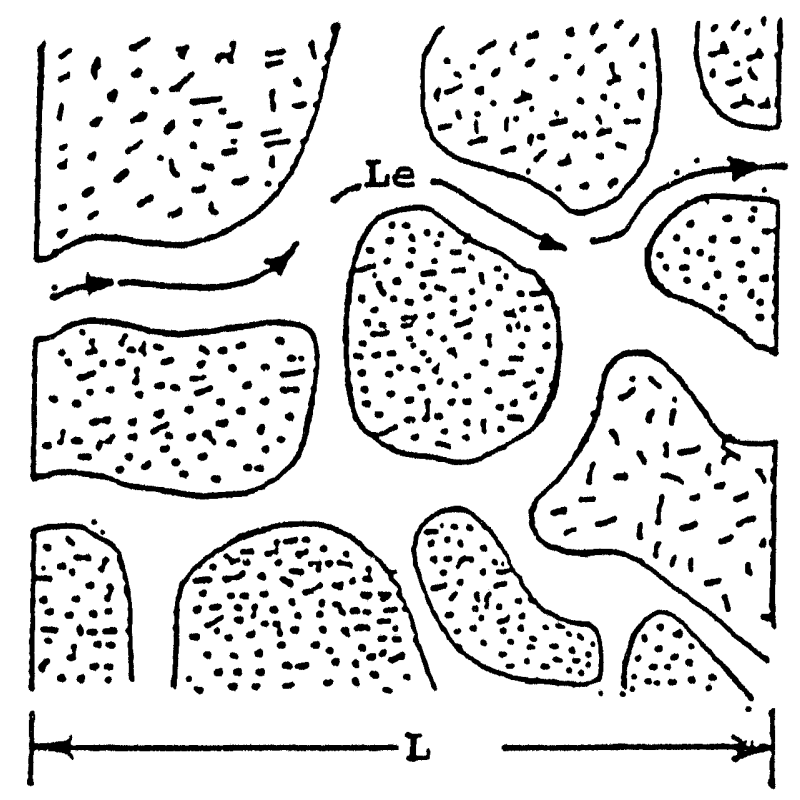

Figure 3.1: Illustration of a tortuous path in a porous medium (Walsh and Bruce, 1984) 


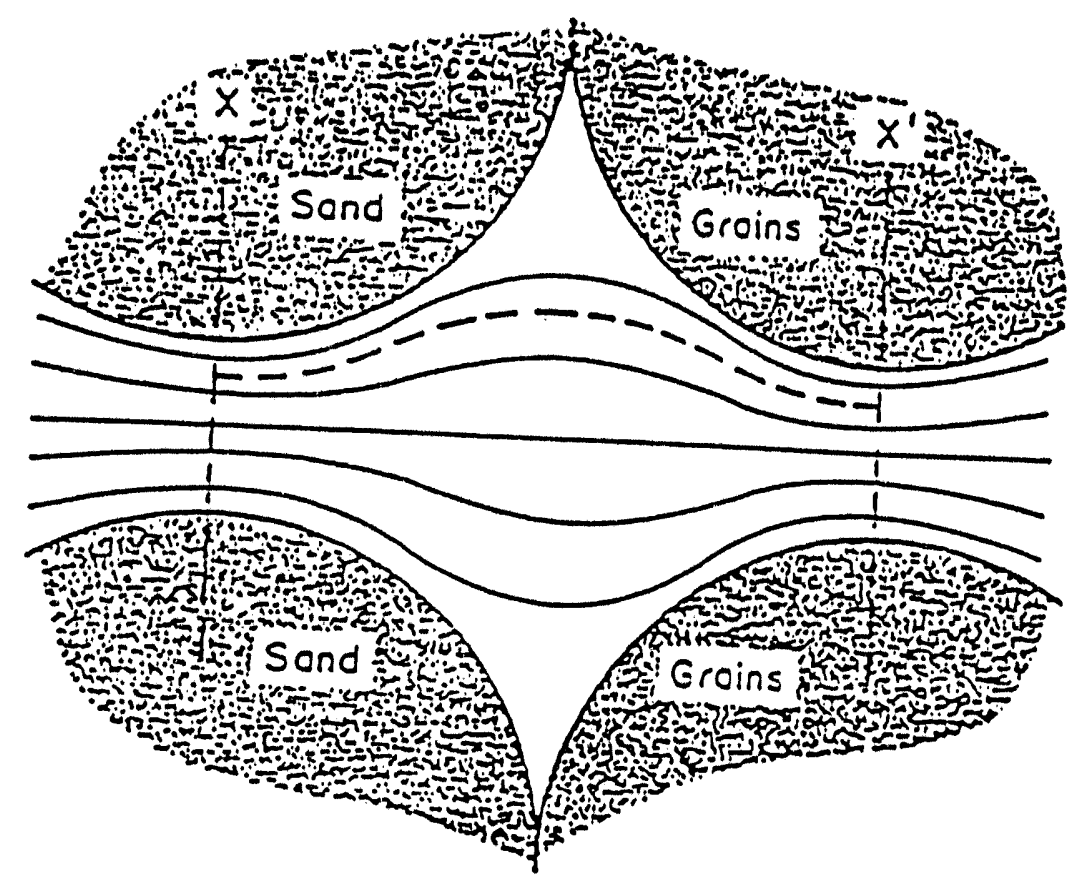

Figure 3.2: Variation of the cross-sectional area of a tortuous path (Winsauer et al., 1952) 
$\mathbf{x}^{\prime}$ will be somewhat longer than the direct distance between the planes. The heavy dotted curved line in Figure 3.2 illustrates this "average" path. Thus, the occurrence of constrictions and enlargements in a pore channel leads to the tortuosity being greater than unity (Winsauer et al., 1952).

By considering the variations of the tortuous path in both the horizontal dimension and the vertical dimension, the overall effect of tortuosity in electric ions or fluid flow should be a power law relationship. To account for the effect of tortuosity on fluid or electric ion flow properties, another parameter, named tortuosity factor, has been arbitrarily defined as

$$
T=\tau^{2}=\left[\frac{L_{c}}{L}\right]^{2}
$$

However, by correlating the measured formation factor and the independentlymeasured tortuosity (measurement methods will be described below), Winsauer et al. (1952) obtained a slightly different form of the correlation as

$$
T=\tau^{1.67}=\left(\frac{L_{e}}{L}\right)^{1.67}
$$

The difference between the powers to the tortuosity in the above two equations is possibly due to the effect of the electrical double-layer (see Figure 3.3) on the electrical measurement of formation factor. The presence of the electrical double-layer in porous media results in a lower formation factor and therefore, the lower power to the tortuosity.

\subsubsection{Measurement of Tortuosity}

It has been shown that tortuosity is a result of certain simplifying assumptions on the pore structure. It is a fundamental property of the streamlines in the conducting capillaries. A knowledge of tortuosity would be instrumental to correlating rock properties. From the definition, it can be measured by having some particles passing through the porous medium. Based on the different measurement mechanisms, there are five available techniques that have been developed. These techniques are: (1) ionic transit time, (2) molecular diffusion, (3) fluid flow, (4) electrical conductance, and (5) acoustic wave propagation. Only fluid flow measurement and electrical measurement techniques are described in this section. 

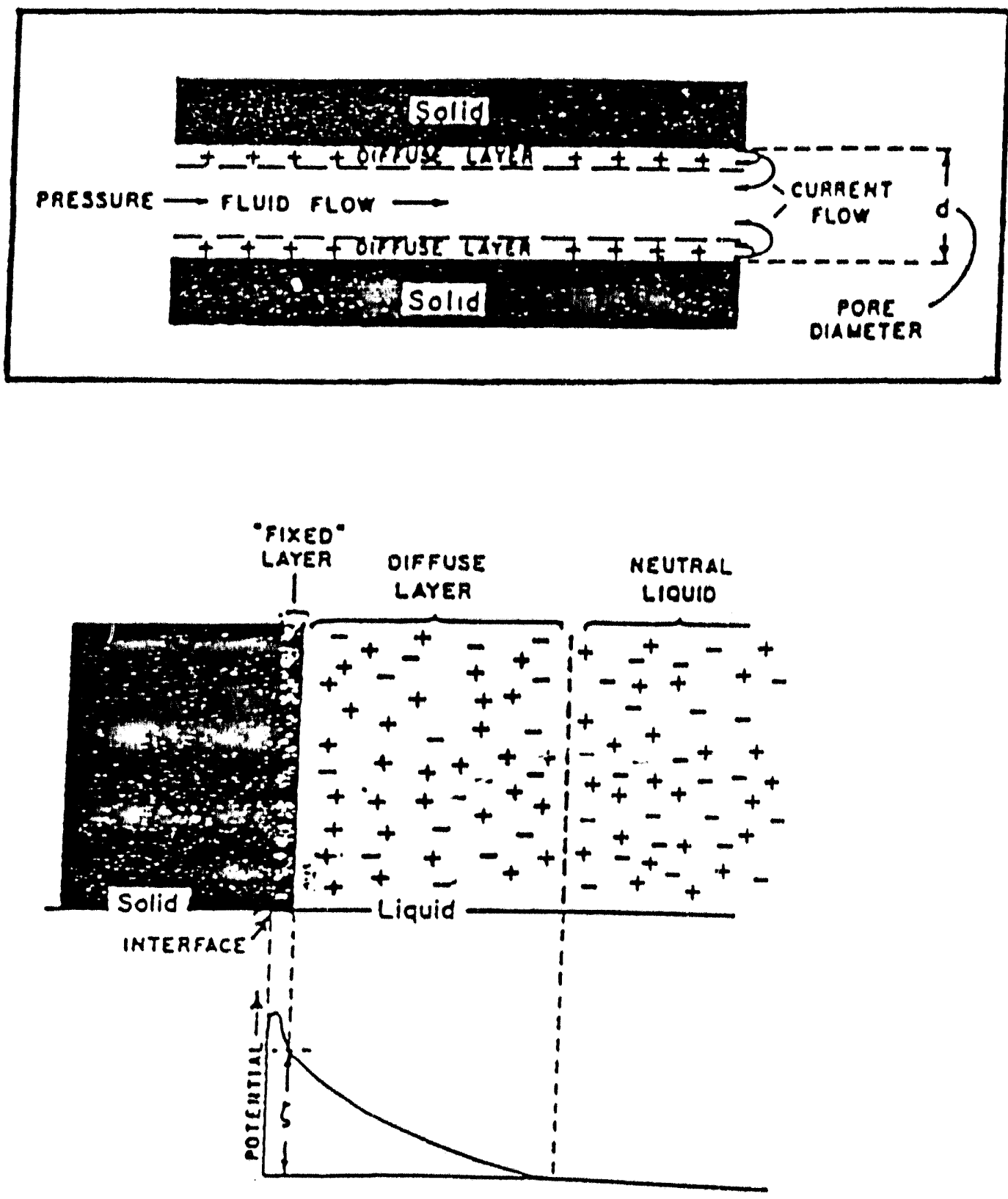

Figure 3.3: Illustration of the electrical double-layer on the interface of solid and fluid phases in a porous medium (Serra, 1984) 


\subsubsection{Fluld Flow Measurement}

This technique is based on the Kozeny-Carman equation (Detailed derivation will be discussed later):

$$
R=\frac{\epsilon}{36 k_{0}} \frac{\phi^{3}}{(1-\phi)^{2}} \frac{d_{p}^{2}}{\tau^{2}}
$$

where:

c = surface factor which is unity for a sphere and less than unity for all nonspherical shapes, and

$k_{0}=$ shape factor which ranges from 2 to 3 , usually 2.5 is assumed.

For a given homogeneous porous medium, particle diameter and porosity are usually known; according to the above equation, the tortuosity can be determined by measuring fluid flow permeability:

$$
\tau=\frac{d_{p}}{6}\left[\frac{\phi}{1-\phi}\right] \sqrt{\frac{\epsilon}{k_{0}} \frac{\phi}{K}}
$$

The tortuosity determined by this method is usually called the "hydraulic tortuosity", to differentiate from the "electrical tortuosity" which is determined from electrical measurement (Scheidegger, 1972).

\subsubsection{Electrical Measurement}

The most common practice in determining tortuosity is by electrical measurement. Archie (1942) defined an electrical parameter, named formation factor, as follows:

$$
F=\frac{R_{0}}{R_{w}}
$$

where:

$$
\begin{aligned}
& F=\text { formation factor, } \\
& \mathbf{R}_{0}=\text { resistivity of a fully saturated rock, and } \\
& \mathbf{R}_{w}=\text { resistivity of the saturating fluid. }
\end{aligned}
$$

For a porous medium, J3q.(3.6) can be expressed as: (Detailed derivation will be discussed later in this chapter)

$$
F=\frac{\tau^{2}}{\phi}
$$

Then the tortuosity is casily calculated from Bq.(3.7) by measuring the formation factor 
and the porosity of the medium.

Since electrical measurement is very easy to perform in the laboratory, this technique is usually used in the industry for measuring tortuosity of a porous medium. But, when the conductivity of the saturation fluid is relatively low, the excess conductivity caused by the double-layer on the interface of fluid and solid (Figure 3.3) cannot be neglected. The electric tortuosity would be under measured. To reduce the effect of the electrical double-layer, the saturation fluid having a sufficiently high conductivity should be used. Bikerman (1940) has shown that the contribution of surface conductance (caused by electrical double-layer on the interface) to the overall conductance can be expressed as a function of salt concentration in solution:

$$
\frac{C_{t}}{C_{t}}=f\left(\frac{1}{\sqrt{C_{\text {sat }}}}\right)
$$

where:

$C_{1}=$ surface conductance caused by the electrical double-layer,

$C_{1}=$ overall conductance, and

$C_{w a}=$ salt concentration (salinity) in the saturation fluid.

Equation (3.8) says when fluid salinity approaches infinity, the contribution of the surface conductance approaches zero. Detailed discussion about the surface conduction of double-layer can be found in references by Winsauer and McCardell (1953), Rink and Schopper (1974), Bvers and Iyer (1975), and Serra (1984). This excess conductance due to ionic double-layer was falsely interpreted as the effect of the "conductive solids" in porous media by Patnode and Wyllie (1950) and de Witte (1950).

\subsection{Rock Properties in Terms of Tortuosity}

\subsubsection{The Tortuous Capillaric Model for a Porous Medium}

One could expect fluid flow through a porous medium to be described by a suitable solution of the Navier-Stokes equations. This could be done if one could formulate correctly the boundary conditions at the highly irregular boundaries. Since it is impossible to define the complicated geometry of the solid surface of the porous medium in any exact mathematical form, one cannot treat the problem in any microscopically exact manner. Fortunately, this difficulty may be circumvented by replacing the porous medium by a simplified fictitious model (Bear, 1969). This model will serve for the purpose if it (a) explains the problem on hand, (b) involves parameters that can be measured and then related to the corresponding properties of the porous medium, and (c) can be treated by currently available mathematical tools to yield a macruscopic description of the problem with discrepancies that can be ignored for practical purposes. 
Intuitively it appears that all rock properties including geometric, fluid flow, electrical, acoustic wave propagation, and some other properties are tied with the parameter of tortuosity of the medium. This fact indicates that a single tortuous capillaric model might suit the problem. Figure 3.4 illustrates the capillaric model for the porous medium.

According to Slawinald (1926), the pore volume of tortuous capillary should be equal to the total pore volume of the medium:

$$
L_{c} * A_{c}=L_{A \phi}
$$

that is

$$
A_{e}=\phi\left[\frac{L}{L_{e}}\right] A=\left(\frac{\phi}{r}\right] A
$$

where:

$A_{1}=$ area available to fluid flow, and

$A=$ cross-sectional area of the porous medium.

Notice that $A_{1}$ is different from $A_{1}$ which is the cross-sectional pore area of the porous medium $(A \phi)$.

\subsubsection{Electrical Propertles of a Porous Medium}

\subsubsection{Formation Factor versus Tortuosity}

An important electrical parameter for characterizing a reservoir rock is the formation factor which is defined by Archie (1942) in Eq.(3.6). Applying Ohm's law to the porous medium having a tortuous flow path as illustrated in Figure 3.4, the formation factor can be expressed as:

$$
F=\frac{\left(\frac{L_{c}}{L}\right)}{\left(\frac{A_{0}}{A}\right)}
$$




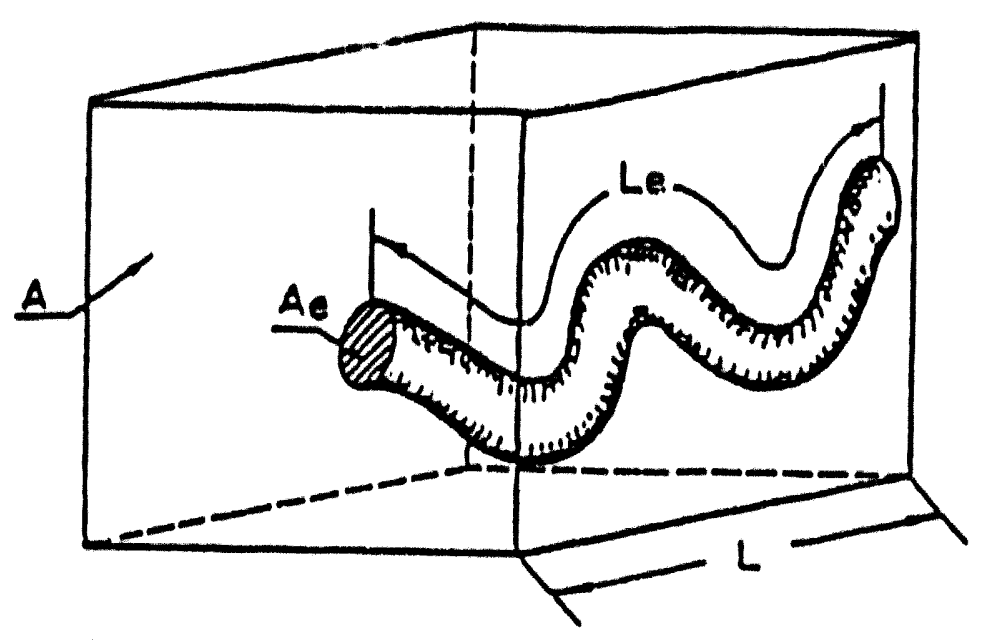

(a)

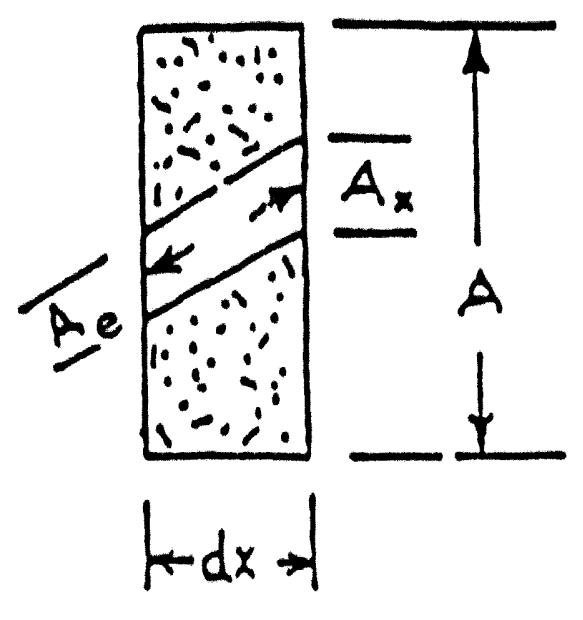

(b)

Figure 3.4: Illustration of the tortuous capillaric model for a porous medium ([a] from Pfannkuch, 1972; [b] from

Walsh and Brace, 1984) 
Substituting Bq.(3.10) into Bq.(3.11) yiolds

$$
F=\frac{\left[\frac{L_{c}}{L}\right)^{2}}{\phi}=\frac{r^{2}}{\phi}
$$

This is the equation (Eq.(3.12)) for determining tortuosity by electrical measurement. However, regarding the area available for flow (A, Wyllie and Rose (1950) assumed that it is

$$
A_{0}=\phi A
$$

Therefore, they arrived at an erroneous expression for the formation factor as:

$$
F=\frac{\left(\frac{L}{L}\right)}{\phi}=\frac{\tau}{\phi}
$$

and their subsequent analyses (Wyllie and Spangler, 1952; Wyllie and Gregory, 1955). Obviously, Wyllie and his co-workers did not differentiate between $A_{x}$ and $A_{1}$, leading to errors in their expressions. Cornell and Katz (1953) corrected this mistake and arrived at the correct expression for formation factor, Eq.(3.12).

\subsubsection{Fluid Flow Properties of a Porous Medium}

Poiseuille's law can be written in the form

$$
u_{0}=-\left(\frac{r_{h}^{2}}{k_{d \mu}}\right) \frac{d p}{d x}
$$

where:

$u_{1}=$ over the cross-section average velocity along a tortuous path, $\mathrm{dp} / \mathrm{dx} \mathrm{x}_{1}=$ pressure gradient along the tortuous path, and

$r_{h}=$ hydraulic radius which is the ratio of the volume of the tortuous path to its wetted area.

As illustrated by Figure 3.4(b), the following relationships hold 


$$
\frac{d p}{d x}-\frac{1}{r} \frac{d p}{d x}
$$

and

$$
u_{1}=\left(\frac{r}{\phi}\right) v=\left(\frac{r}{\phi}\right)\left(\frac{Q}{A}\right)
$$

where $\mathrm{dp} / \mathrm{dx}$ is a pressure gradient along the porous medium, and $\mathrm{Q}$ is a flow rate. Further, according to Darcy's law, the fluid permeability of the porous medium is defined as

$$
R=-\frac{\left(\frac{g}{A}\right)^{\mu}}{\left(\frac{d p}{d x}\right)}
$$

Substituting Bqs.(3.15) to (3.17) into Bq.(3.18) yields

$$
K=\frac{r_{h}^{2}}{k_{0}} \frac{\phi}{2}=\frac{r_{h}^{2}}{k_{\sigma} F}
$$

Furthermore, considering the definition about the mean hydraulic radius $\left(r_{b}\right)$ given by Carman (1956), it is

$$
r_{h}=\frac{\phi}{S}
$$

and

$$
S=S_{0}(1-\phi)=\frac{6}{c d_{p}}(1-\phi)
$$

where $S=$ particle surface area per unit bulk volume, and $S_{0}=$ specific surface area of the particles. Substituting Bqs.(3.20) and (3.21) into Bq.(3.19), the following equation for permeability is derived

$$
K=\frac{\epsilon}{36 k_{0}}\left(\frac{\phi}{1-\phi}\right)^{3} \frac{d_{p}^{2}}{F}
$$

This equation can also be expressed in terms of tortuosity by substituting Eq. (3.12) into Bq. (3.22): 


$$
X=\frac{e}{36 k_{0}} \frac{\phi^{3}}{(1-\phi)^{2}} \frac{d_{p}^{2}}{\tau^{2}}
$$

This is a different form of the Kozeny-Carman equation which relates fluid flow property (i.e., permeability) to the electrical property (i.e., formation factor or tortuosity). Similar results have been derived by Paterson (1983) and Walsh and Brace (1984).

\subsubsection{Dispersion Process in a Porous Medium}

By applying the basic dispersion equation to the tortuous capillaric model illustrated in Figure 3.4, the following equation for describing dispersion phenomena in a tortuous capillary can be derived:

$$
D_{L}^{\prime} \frac{\partial^{2} C}{\partial x_{s}^{2}}-V^{\prime} \frac{\partial C}{\partial x_{e}}=\frac{\partial C}{\partial t}
$$

with

$$
D_{L}^{\prime}=D\left(\frac{r^{2}}{\phi}\right)
$$

where $V^{\prime}=$ real pore velocity, and $D_{L}^{\prime}=$ dispersion coefficient in the tortuous capillary. Obviously, Eq.(3.24) is similar to Eq.(2.2) in form. Therefore, it can be solved in the similar way as Eq.(2.2):

$$
C=\frac{1}{2} \operatorname{ergc}\left(\frac{x_{i}-V^{\prime} t}{\sqrt{4 D_{L}^{\prime} t}}\right)
$$

Substituting Eqs.(3.25) and (3.26) into Eq.(3.27), exactly the same solution equation as Eq. (2.4) is obtained:

$$
C(x, t)=\frac{1}{2} \operatorname{erfc}\left(\frac{x-v_{\ell}}{\sqrt{4 D_{L} t}}\right)
$$

Therefore, the dispersion theories described in Chapter 2 can be applied directly to the tortuous capillaric model of porous media.

Similarly, the dispersion-capacitance model can also prove to be applicable to the tortuous capillaric model of a porous medium. 


\subsection{Relating DLspersivity to Rock Parameters}

As pointed out in Chapter 2, Raimondi ef al. (1959) have given a general form of the relationship between dispersion coefficient, grain size, and velocity in this form:

$$
D_{L}=D_{m}+c \sigma_{H} v_{c} d_{p}
$$

From this equation (Bq.(3.29)), the dispersivity can be mathematically defined as

$$
\alpha_{D}=\frac{D_{L}-D_{m}}{V_{c}}=c \sigma_{2} d_{p}
$$

In a more general form, Eq. (3.30) should be written as:

$$
\alpha_{D}=\cos _{H}^{\prime} d_{p}^{\prime}
$$

where $p$ and $q$ are correlation exponents.

Solving the particle diameter from Eq. (3.23), we have

$$
d_{p}=6 r\left(\frac{1-\phi}{\phi}\right) \sqrt{\frac{k_{0}}{6} \frac{R}{\phi}}
$$

Substituting Bq.(3.32) into Bq.(3.31) yields

$$
\alpha_{D}=\operatorname{co}_{H}^{p}\left[6 r\left(\frac{1-\phi}{\phi}\right) \sqrt{\frac{k_{0}}{\epsilon} \frac{K}{\phi}}\right]^{\prime}
$$

This is the equation that relates dispersivity to other rock property parameters. It indicates that if the rock property parameter such as porosity, tortuosity, permeability, and heterogeneity can be measured by or derived from well logs, the in-situ dispersivity of reservoir rocks can then be evaluated by Eq. (3.33).

In general, $p$ and $q$ are assumed to equal $1.0, k_{0}=2.5, \epsilon=1.0$, and $K$ in millidarcy ( $1 \mathrm{md}=0.869 \times 10^{-12} \mathrm{~cm}^{2}$ ). Substituting these values into Eq.(3.33) yields the practical equation as: 


$$
\alpha_{D}=2.98 \times 10^{-s} \operatorname{co}_{H^{\top}}\left(\frac{1-\phi}{\phi}\right) \sqrt{\frac{K}{\phi}}
$$

or expressed in terms of the formation factor as

$$
\alpha_{D}=2.98 \times 10^{-3} c \sigma_{M}\left(\frac{1-\phi}{\phi}\right) \sqrt{K F}
$$

where $\mathrm{K}$ is in millidarcy units.

Since the above derivation process is based on the single-phase fluid flow, the conclusion equation 3.35 is only valid for the single-phase miscible displacement systems. The next chapter will be a discussion of how to determine those rock properties, i.e., the parameters of the equation 3.35, from well logs. 


\section{CHAPTER 4}

\section{DETERMINATION OF ROCK PROPERTIES FROM WELL LOGS}

In the preceding chapter, the relationships between dispersivity and rock property parameters have been established. In those equations the rock property parameters such as porosity, tortuosity, permeability, and heterogeneity are included. Since one of the goals of this study is to determine the in-situ dispersivities of reservoir rocks from well logs, this chapter will focus on the techniques for determining those rock property parameters from well logs. However, since most of the log analysis techniques are available in the literature, only a brief discussion is presented in this chapter.

\subsection{Pre-Treatment of Well Log Data}

Since well logging is conducted after an interruption (or the termination) of drilling activity, the log readings will include rock properties plus borehole environments. Also all $\log$ tools need to be calibrated each time before use. Due to the different conditions at each time and human variations, the calibration for the same type of tool might have different errors involved. To obtain the logging parameters that accurately depict the reservoir, it is necessary to remove the effects caused by borehole conditions and improper tool calibration. To do this, the following two steps must be carried out: (1) the log readings must be environmentally corrected, that is, adjusted for borehole conditions, and (2) the log readings must be normalized.

These two procedures are vital to any petrophysical analysis using well log data. It must be pointed out that normalization must be tempered with discretion, so that reservoir characteristics will not be mistaken for calibration errors.

\subsection{Determination of Porosity}

Porosity-measuring log tools have been well developed. Currently, there are three porosity-measuring tools in common use --- Density, Neutron, and Sonic. There are several tools for one parameter because all porosity-measuring log tools respond not only to the porosity but also to the lithology of the rock and to the type of fluid filling the pore space. When the lithology of the rock and the type of fluid in the pore space are known, one porosity-measuring tool can provide the accurate porosity. In other cases more than one porosity-measurement is needed to sort out the parameters.

The Sonic log used to be the popular porosity tool because it was less sensitive to borehole and mud-cake variations than the early Density and Neutron tools. However, 
in recent years the Density-Neutron combination has replaced the Sonic log as the primary source of porosity information. There are several reasons why the Densityneutron combination is preferred to the Sonic log. These reasons are well summarized by Dewan (1983) as follows:

- Porosity can be determined without precise knowledge of the rock matrix,

- There is no need for the compaction correction required with Sonic porosity,

- Overlay of Density-Neutron curves is an excellent gas indicator,

- Transitions from one type of rock matrix to another can often be distinguished, and

- Shale effects are more evident and can be accounted for more precisely.

Consequently, the Sonic log becomes a backup porosity tool to be used where the Density or the Neutron log fails. In general, the Sonic log is needed where the hole is very irregular, where the hole is filled with air, where secondary porosity is important, or where heavy minerals (e.g. pyrite) are present.

\subsection{Determinations of Formation Factor and Tortuosity}

\subsubsection{Mud Fluid Invasion Profile}

It is well known that during mud drilling the hydrostatic pressure in the borehole must be kept greater than the reservoir pressure to prevent a well blowout. The differential pressure, therefore, forces drilling fluid into the formation. As this happens, solid particles in the drilling fluid plate out on the formation wall and form a mud cake. Liquid that filters through this mud cake (i.e., the mud filtrate) passes into the formation and displaces some of the reservoir fluid. An invaded zone is thus formed adjacent to the wellbore. This invaded zone is further arbitrarily subdivided into the flushed zone and the transition zone as illustrated in Figure 4.1. Further, due to the difference in density and mobility of water and oil, the invaded profiles in wet zone (water-bearing) and pay zone (hydrocarbon-bearing) are different; as a result, the electric log responses are also different (see Figure 4.2).

\subsubsection{Absolute Tortuosity from Micro-log}

The micro-log (or spherical-focused log) is designed to measure the resistivity of the flushed zone. Generally the flushed zone is assumed to contain 100 percent muci filtrate. Therefore, the micro-log readings can be used directly to evaluate the formation factor and the absolute tortuosity by the following equations:

$$
F=\frac{R_{S F Z}}{R_{m}}
$$




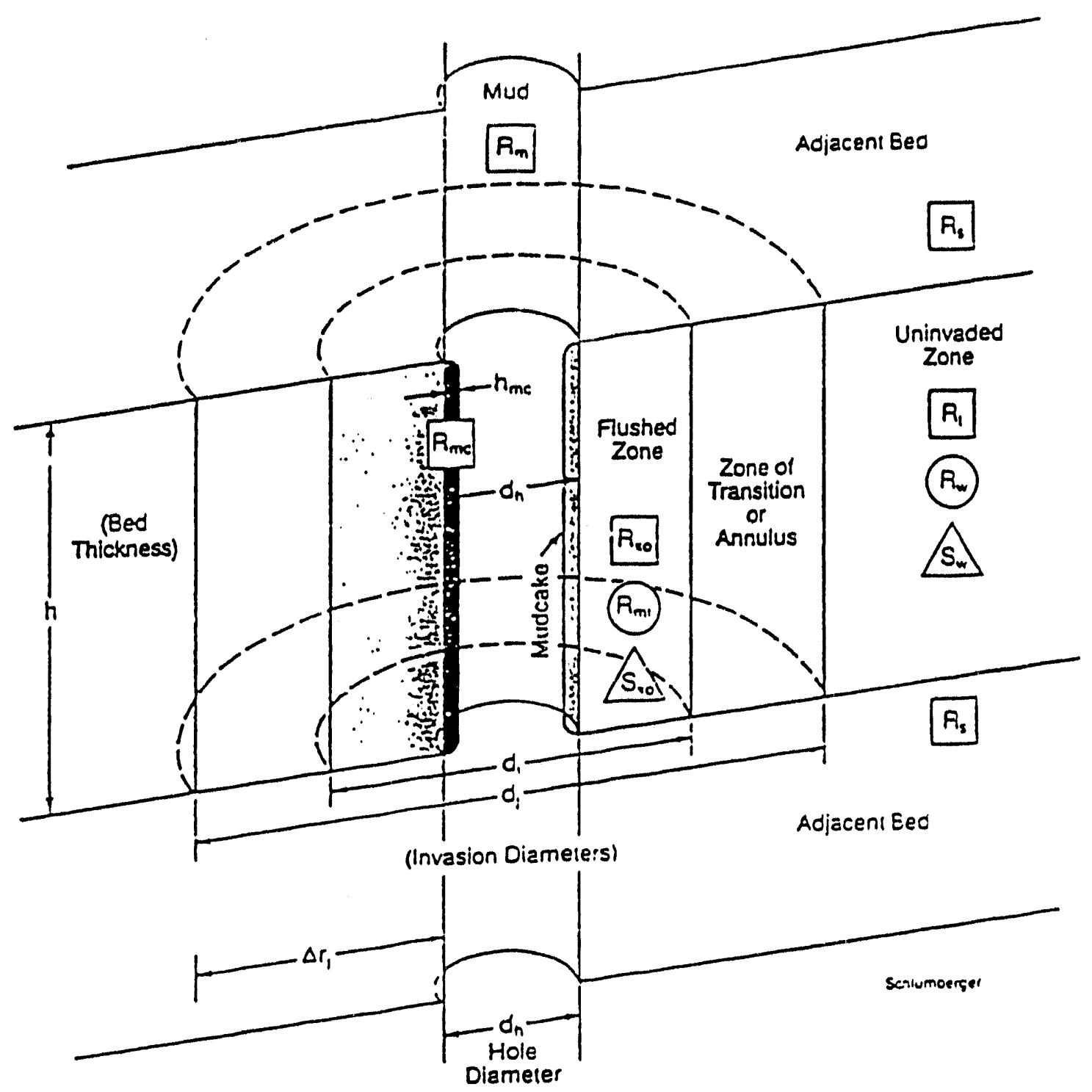

Figure 4.1: Mud fluid invasion profile (Schlumberger, 1991) 
(a) INVADED WET ZONE
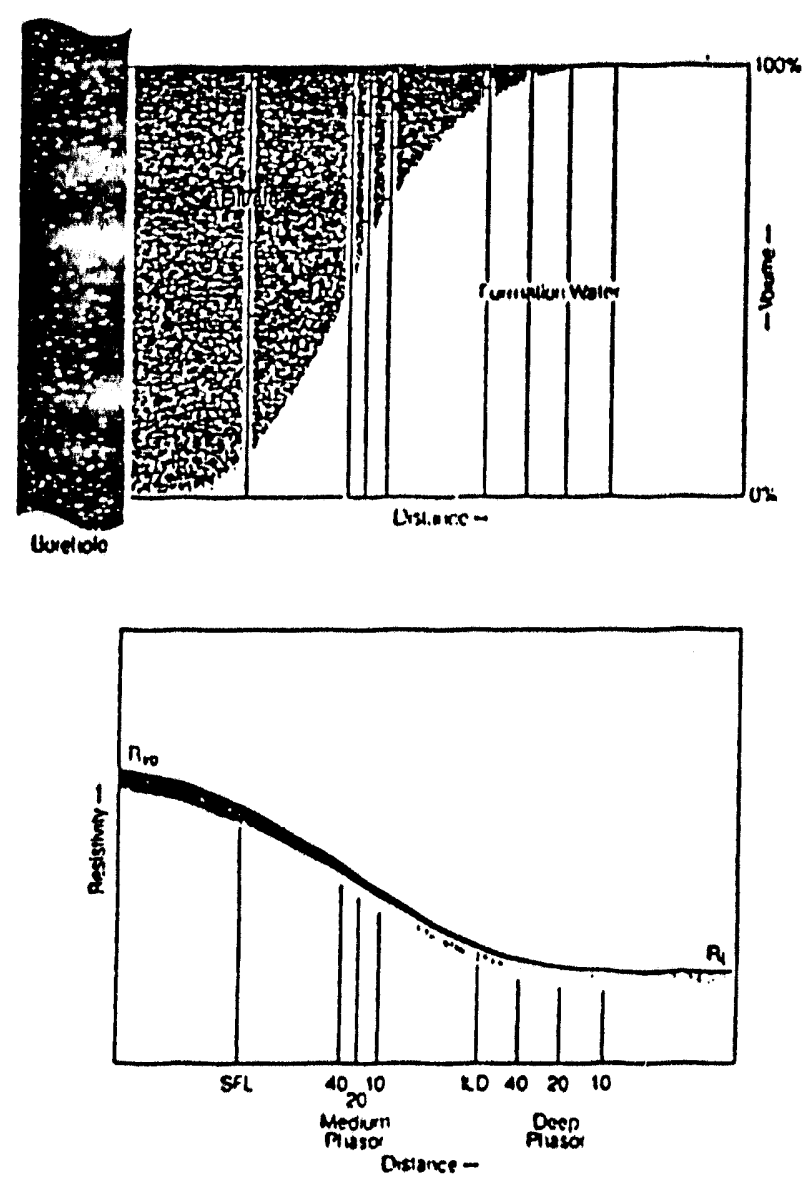

(b) INVADED PAY ZONE
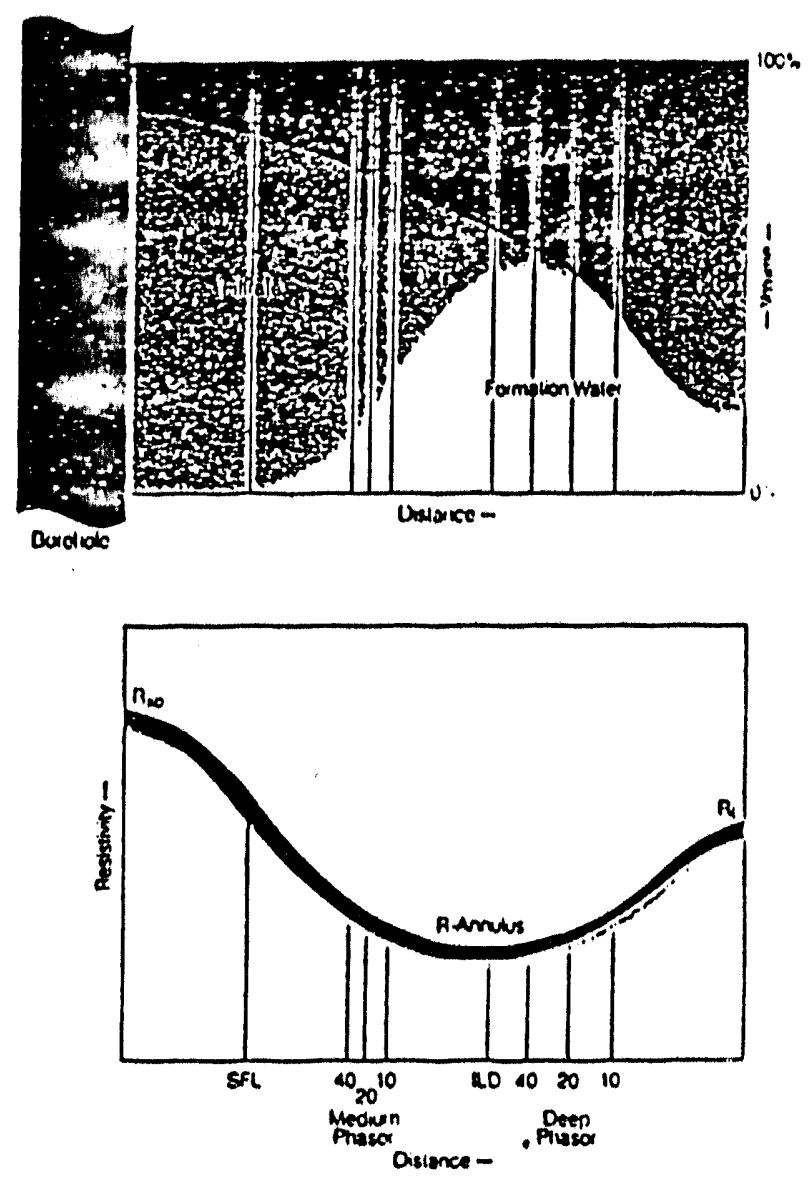

Figure 4.2: Electric log responses in mud fluid invaded formations (Schlumberger, 1990) 
and

$$
\tau=\sqrt{F \phi}=\sqrt{\left[\frac{R_{s z}}{R_{w l}}\right]_{\phi}}
$$

where $\mathrm{R}_{\mathrm{gFL}}=$ the micro-log resistivity, and $\mathrm{R}_{\mathrm{mf}}=$ mud filtrate resistivity.

\subsubsection{Undisturbed Formation Resistivity and Saturation}

To determine the original reservoir saturation, the undisturted formation resistivity must be determined. This can be accomplished with either Dual Induction or Dual Laterolog.

Dual resistivity measurements make invasion correction possible. However, invasion is not as easy as it looks because the invasion profile is not a piston-like front (see Figure 4.2). Some "tornado" charts for invasion correction are usually available from the logging service company. Chen et al. (1983) developed a new method for invasion corrections using the tool calibration coefficients, instead of the geometrical factors in the convectional methods. The equation is

$$
R_{t}=\left[\frac{K_{d}}{\overline{K_{d}-K_{d}}}\right] R_{d}-\left(\frac{K_{d}}{\overline{K_{d}}-\bar{K}_{d}}\right) R_{d}
$$

where:

$R_{d}=$ resistivity reading from the deep-investigating logging tools,

$R_{1}=$ resistivity reading from the shallow-investigating logging tool,

$K_{d}=$ the calibration coefficient of the deep-investigating logging tool, and

$\mathrm{K}_{\mathbf{q}}=$ the calibration coefficient of the shallow-investigating logging tool.

In a separate paper, Chen et al. (1985) further derived the equation for computing the tool calibration coefficient from the tool configuration:

$$
K_{d}=\frac{2 \pi h_{d}}{\ln \left[\frac{2 L_{d}}{d_{0}}\right]}
$$




$$
K_{1}=\frac{2 \pi h_{0}}{\ln \left(\frac{2 L_{d}}{d_{0}}\right)}
$$

where:

$h_{4}=$ the average thickness of the measuring electric current layer of the deep investigation tool,

$h_{1}=$ the average thickness of the measuring electric current layer of the shallow investigation tools,

$d_{0}=$ outer diameter of the tool,

$L_{d}=$ distance from wellbore to the equivalent zero potential point for the deep investigating tools, and

$L_{1}=$ distance from wellbore to the equivalent zero potential point for the shallow investigating tools.

The average thickness of the measuring electric current layer is approximately equal to (slightly greater than) the vertical resolution of the tool. The equivalent zero potential distance from the borehole is approximately equal to the distance from the transmitting electrode to the receiving electrode.

Equation 4.1 usually gives very good results when the invasion diameter is less than the depth of investigation of the shallow or medium $\log$ tool. When the invasion diameter is greater than the depth of investigation of the shallow log tool, Bq.(4.3) insufficient correction for the invasion effect results. In these cases, some other complementary approaches such as "tornado" charts should be used.

With the true resistivity of the undisturbed formation and the micro-log resistivity, the relative resistivity can be then determined by equation

$$
R_{r w}=\frac{R_{s m}}{R_{t}}
$$

Using the relationships in the last chapter, the water saturation is further calculated. If a water zone is available in the formation profile, the above results can be cross-checked by the following relationship:

$$
\frac{R_{s p z}}{R_{m v}}=\frac{R_{r}}{R_{w}}
$$

where $R_{w}$, the formation water resistivity, can be easily determined from the SP log. If the SP $\log$ is not available, the above equation can also be used to estimate $R_{w}$. 


\subsection{Estimation of In-Situ Permeability}

Estimation of in-situ permeability from well logs has been a goal of the log analyst for many years. Tremendous work has been done during the past four decades. As a result, soven methods have been developed for obtaining permeability from well log measurements (including formation testers). These seven methods are:

(1) permeability from electric logs,

(2) permeability from porosity logs,

(3) permeability from the combination of electric and porosity logs,

(4) permeability from nuclear magnetism logs,

(5) permeability from geochemical logs,

(6) permeability from acoustic logs, and

(7) permeability from repeated formation testers (RFT).

Among these seven methods, $\# 7$ usually gives the most accurate permeability values. However, due to the time and cost involved and the non-continuous measurements, the formation tester is not used as widely as some other methods. At present, \#3 method is the most widely used in the industry. All the methods have been summarized in the references by Ahmed $a t$ al. (1989) and Bums (1991). For later uses, the method \#3 is presented here.

The original equation that relates permeability with porosity and internal surface area is the Kozeny-Carman equation (Bq.(3.23)). Due to the difficulty in determining the internal surface area, many investigators have tried to relate the surface area to the connate water saturation which can be determined from the electric logs. First, Wyllie and Rose (1950) gave the form of the equation as:

$$
R=c \frac{\phi^{3}}{S_{w}^{2}}
$$

where $c$ is a constant.

Later, Timur (1968) generalized this equation by comparing the core data with loganalysis data: 


$$
R=c \frac{\phi^{a}}{S_{w}^{b}}
$$

where a, b and $\mathrm{c}$ are corrolation constants. According to the analyses on 155 sandstone samples from the different oil fields in North America, Timur found $c=0.136, a=4.4$, and $b=2$. Substituting these values into Bq.(4.9), it becomes

$$
R=0.136 \frac{\phi^{4.4}}{S_{w}^{2}}
$$

This equation applies to a wide range of sand permeability from $0.1 \mathrm{md}$ to 10,000 md. The fitting of the equation with the core permeability data has a very strong correlation coefficient $(0.97)$ and a standand error of 0.314 orders of magnitude. However, this equation is only valid for granular porous media. The correlation constants in Eq. (4.10) are not universal values. For a particular type of reservoir, these constants should be determined through correlation analyses of laboratory core data and field well $\log$ data.

\subsection{Determination of Heterogeneity Factor}

\subsubsection{Defining Heterogenelty Factor in Terms of Permeabllity Variation}

The heterogeneity factor, in a general sense, is a term which describes the degree of spatial variation of a characteristic in a substance (Lambert, 1981). Some heterogeneous reservoir characteristics include permeability, porosity, pore size distribution, and fluid saturation. Since this work deals with fluid flow behavior in sandstone rocks, reservoir heterogeneity will be defined in terms of the variation of permeability distribution in a reservoir.

Koval (1963) defined a heterogeneity factor (H) in terms of the Dykstra-Parsons permeability variation $\left(V_{D P}\right)$ as shown in Figure 4.3. From this figure, an approximate expression for the relationship between the heterogeneity factor and the Dykstra-Parsons permeability variation is obtained as:

$$
H=e^{\left(0.25 V_{p D}\right)}
$$

where; $H=$ Koval's heterogeneity factor, and $V_{D P}=$ Dykstra-Parsons permeability variation. Based on this relationship, the reservoir heterogeneity factor can be determined from the known permeability variation coefficient. 


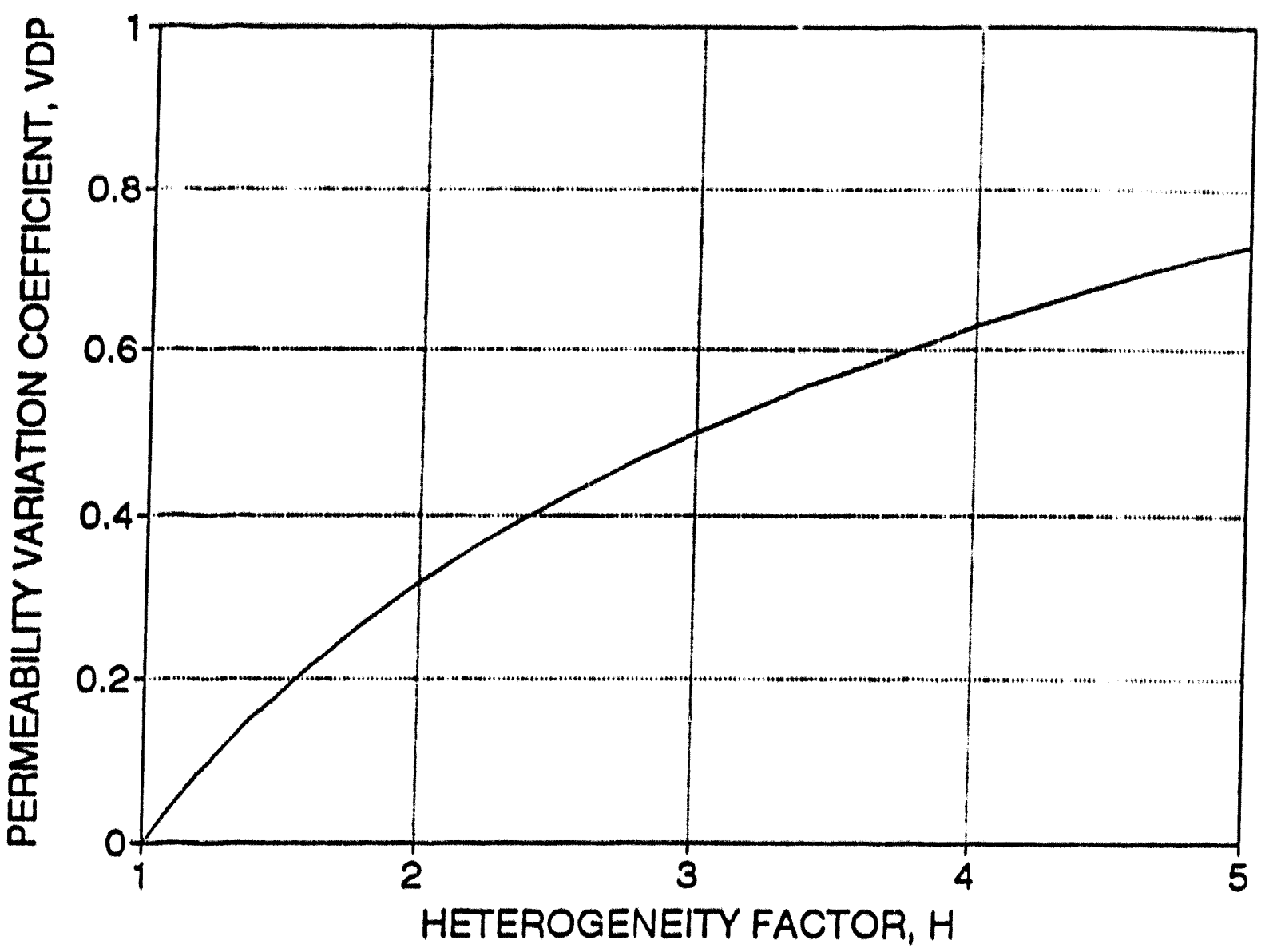

Figure 4.3: Relationship between heterogeneity factor and Dykstra-Parsons permeability variation (from Koval, 1963) 


\subsubsection{Determining Permeabllity Varlation Coefricient}

The study of the permeability distribution began with Law's (1944) work, in which he concluded that permeability may be log-normally distributed. Since then, the permeability distribution has generally been accepted to be log-normal.

To determine the permeabllity vartation, investigators have usually resorted to one of two statistics: the Lorenz coefficient $V_{L Z}$ or the Dykstra-Parsons coefficient $V_{D P}$ (Dykstra and Parson, 1950). Through a complete statistical study, Jensen (1986) proposed to use two parameters for characterizing the permeability variation. However, since we will use the relationship between the heterogeneity factor and the permeability variation coefficient which was developed by Koval (1963), the Dykstra-Parsons permeability variation coefficient will be used in this study. The relationships between the heterogeneity factor and the other two permeability variation coefficients are reserved for future studies.

Based on the assumption of a log-normal distribution of permeability, Dykstra and Parsons (1950) proposed to determine the permeabillty variation by the following procedure:

(1) Arrange the permeability values in descending order of magnitude over equal intervals of porhaps one foot of formation.

(2) Calculate the percentage of the property magnitude exceeding each tabulated entry to obtain the "cumulative percent greater than".

(3) Construct a plot of the data as presented in the previous steps on a log probability coordinate graph paper.

(4) Draw a best fit straight line through the plotted data, with the contral points weighted more heavily than the more distant end points.

(5) Calculate the permeability variation coefficient by the following equation:

$$
V_{D P}=\frac{\boldsymbol{R}_{s 0}-\boldsymbol{R}_{\mathrm{u}, 1}}{\boldsymbol{K}_{s 0}}
$$

where $\mathbf{K}_{s 0}=$ the mean permeability (i.e., the permeability value at the 50 percent probability); and $K_{M .1}=$ the permeability value at 84.1 percent of the cumulative sample.

The permeability variation coefficient determined by Bq.(4.12) ranges from zero to one, with a completely uniform system having a value of zero, and typical oilfield reservoirs having values from 0.6 to 0.8 . 
In addition, it should be pointed out that if the horizontal permeablity distribution data are used in Bq. (4.12), the $V_{D p}$ will be called the horizontal permeability variation coefficient; similarly, for the vertical permeability distribution data, the $V_{D p}$ determined by Eq.(4.12) will be called the vertical permeability vartation coefficient. Accordingly, the heterogeneity factor defined in Bq.(4.11) will be named as the horizontal heterogeneity factor and the vertical heterogeneity factor, respectively. Knowing the porosity, formation factor or tortuosity, permeability, and heterogeneity factor determined from well logs, the rock dispersivity can be calculated using Bq.(3.35). Further discussions on this subject may bo found in chapter 7. 


\section{CHAPTER 5}

\section{LABORATORY EXPERIMENTAL DESIGN AND PROCEDURES}

In the preceding chapters, theoretical correlations between dispersivity and other rock properties have been developed and the determination techniques for the rock properties from well logs have also been discussed. To test these relationships and techniques in the laboratory, laboratory experiment was 20 designed that both dispersivity and rock properties were measured on several groups of sandstone core samples. This chapter presents the experimental design, apparatus, and procodures.

\section{S.1 Preparation of Core Samples}

In this study, twenty-one relatively clean (clay-free) sandstone samples were tested. These samples were classified into five groups. Each group had three to six samples. The first four groups were Berea sandstone which were drilled from four different Berea candstone blocks, and the fifth group was Brown sandstone which was obtained from one single Brown sandstone block. These five sandstone blocks were obtained from five different sources. Therefore, each group of samples should have had a distinct characterlstic.

After cutting and shaping, the samples were extracted of any hydrocartions present using toluene, and leached of any sults present using methanol, in a Soxhlet solvent reflux apparatus. Cleaning of the samples took place by solvent imbibition, diffusion, and drainage. This technique was considered to be effective because of the high permeabilities of the samples (greater than $140 \mathrm{md}$ ). The cleaning process was held for two to three days. Then the samples were placed in an oven for two days. The oven temperature was kept at 95 degrees celsius (203 degrees fahrenheit). The weights of the dried core samples were also recorded after cooling. The geometric descriptions of the samples are listed in Tables 5.1a through 5.1e.

\section{S.2 Coating and Mounting of Core Samples}

Since this study involved fluid flow tests, coating was required to serve as an impermeable boundary allowing fluid to flow through the length of the core. The coating method used here was developed by Menzie ot al. (1988). In this method, an industrial compound named "J-B Weld", a "liquid metal", was applied in a thin layer to the entire outer surface with the exception of the two ends. Since the J-B Weld has a very thick consistency it is expected that its penetration into the pores of core is very limited. More layers of the coating can be applied if higher pressure resistance is 
Table 5.1a: Description of Core Samples (Group \#1)

\begin{tabular}{|l||c|c|c||}
\hline Core Number & Type & $D(\mathrm{~cm})$ & $I(\mathrm{~cm})$ \\
\hline B 12 & Berea & 2.50 & 9.60 \\
\hline B\#2 & Berea & 2.50 & 9.45 \\
\hline B\#3 & Berea & 2.50 & 9.16 \\
\hline B\#4 & Berea & 2.50 & 9.41 \\
\hline B\#5 & Berea & 2.50 & 9.30 \\
\hline B\#6 & Berea & 2.50 & 7.20 \\
\hline
\end{tabular}


Table 5.1b: Description of Core Samples (Group 12)

\begin{tabular}{||l|l|l|l|}
\hline Core NO. & Type & $D(\mathrm{~cm})$ & $I(\mathrm{~cm})$ \\
\hline B*10 & Berea & 2.50 & 9.34 \\
\hline BN11 & Berea & 2.50 & 9.30 \\
\hline B\#12 & Berea & 2.50 & 7.08 \\
\hline
\end{tabular}

Table 5.1c: Description of Core Samples (Group \#3)

\begin{tabular}{|c|c|c|c|}
\hline Core No. & Type & $D(\mathrm{~cm})$ & L (cm) \\
\hline$L B \| 1$ & Berea & 3.80 & 14.65 \\
\hline LB $\# 2$ & Berea & 3.80 & 14.55 \\
\hline$L B \| 3$ & Berea & 3.80 & 14.55 \\
\hline LB $\# 4$ & Berea & 3.80 & 14.10 \\
\hline LB $\# 5$ & Berea & 3.80 & 13.95 \\
\hline
\end{tabular}


Table 5.1d: Description of Core Samples (Group \#4)

\begin{tabular}{|c||c|c|c|}
\hline Core No. & Type & $D(\mathrm{~cm})$ & $L(\mathrm{~cm})$ \\
\hline LB \#6 & Berea & 3.70 & 14.70 \\
\hline LB \#7 & Berea & 3.80 & 14.60 \\
\hline LB \#8 & Berea & 3.80 & 14.30 \\
\hline
\end{tabular}

Table 5.1e: Description of Core Samples (Group \#5)

\begin{tabular}{|l||c|c|c|}
\hline Core No. & Type & $D(\mathrm{~cm})$ & L (cm) \\
\hline \hline BN \#1 & Brown & 2.50 & 9.32 \\
\hline BN \#2 & Brown & 2.50 & 9.52 \\
\hline BN \#3 & Brown & 2.50 & 9.46 \\
\hline BN \#4 & Brown & 2.50 & 6.38 \\
\hline
\end{tabular}


required. Since the tests were performed at the room conditions, two layers of the J-B Weld were sufficient for the present study. After the metal coating mixture was applied to the core, the core was put aside for two days to allow the coating to solidify gradually.

Once the coating material was completely dry, the core sample was mounted in the testing apparatus (see Figure 5.1). This apparatus consisted of two endplates held together by four comer bolts that spanned the length of the core. A gasket of $1 / 8$ " thick rubber was placed between the core ends and the endplates to secure a sea' with the core. The inner hole of the gasket was as wide as possible to allow the greatest contact between the ends of the core and fluid. During the mounting process, the core was carefully centered between the two endplates and was not over-tightened. Once this was finished, the core was ready for the fluid flow and miscible displacement tests. Hereafter the entire core/endplate assembly will be referred to as the "medium".

\subsection{Preparation of Fluids}

\subsubsection{Brine Solution for Electrical Measurement}

To measure resistivities of the core samples, a conducting fluid is needed. Brine was used for this purpose. The brine solution was prepared using the gravimetric technique by adding a weighted amount of distilled water. Its density, viscosity, and electrical resistivity were determined by direct measurements and then cross-checked with the given tables and figures in the literature (see Table 5.2).

\subsubsection{Miscible Fluids for Displacement Test}

To conduct miscible displacement tests for determining the "standard" dispers: vities, two "matched" fluids are necessary. These two fluids must meet the foilowing requirements: (a) completely miscible, (b) equal viscosities and equal densities, (c) wide range of refractive indices, (d) no chemical reactions with rock matrix, no adsorption on rock matrix, (e) easy to get and cost effective, and (f) easy to clean for repeat runs. Based on these considerations, methanol and toluene were selected. As shown in Table 5.3, these two chemicals meet all the above requirements except their densities. However, since our tests were designed for a horizontal position, the effect of the small density difference can be considered negligible.

\subsection{Measurements of Basic Rock Properties}

Basic rock properties measured here include porosity, formation factor, and permeability. Since the techniques for measuring these basic rock properties are standard, the measurement processes are only briefly discussed in this section. Detailed description of these measured techniques can be found in the references by Amyx et al. 


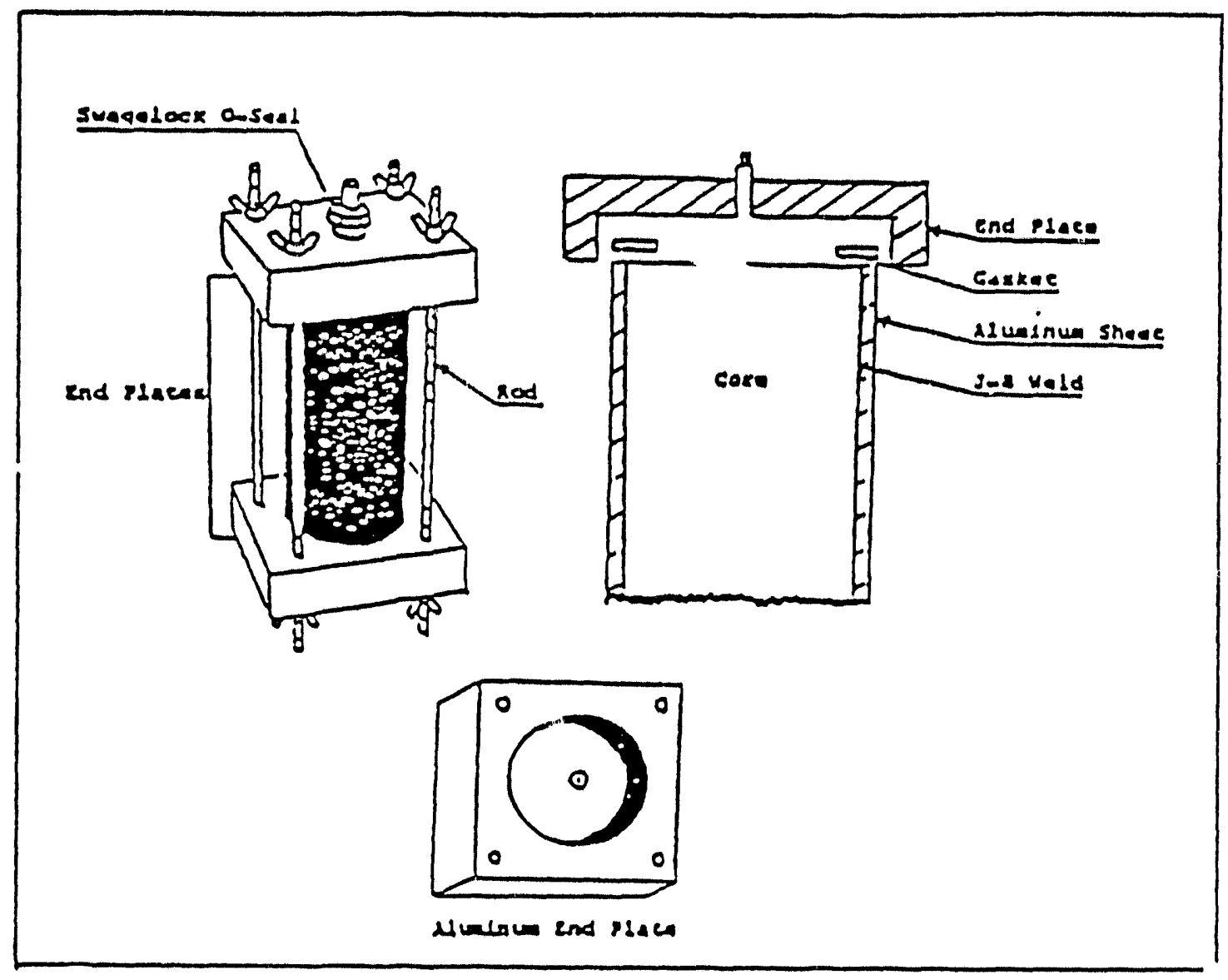

Figure 5.1: "J-B Weld" coated core assembly and cross section (Menzie et al., 1988) 
Table 5.2: Brine Solution Data

\begin{tabular}{|c|c|}
\hline Components: & $3: \mathrm{NaCl}+1 \div \mathrm{CaCl} 2$ \\
\hline salinity: & about $40,000 \mathrm{ppm}$ \\
\hline Density: & e $24^{\circ} \mathrm{C} \& 1 \mathrm{~atm}$ \\
\hline viscosity: & e $24^{\circ} \mathrm{C} \& 1 \mathrm{~atm}$ \\
\hline Resistivity: & $0.19 \mathrm{ohm}-\mathrm{m} \& 24^{\circ} \mathrm{C} \& 1 \mathrm{~atm}$ \\
\hline
\end{tabular}

Table 5.3: Physical Properties of Methanol and Toluene (at room conditions: $24^{\circ} \mathrm{C}$ and $1 \mathrm{~atm}$ )

\begin{tabular}{|l|c|c|}
\hline Properties & Methanol & Toluene \\
\hline Formula: & $\mathrm{CH}_{3} \mathrm{OH}$ & $\mathrm{C}_{6} \mathrm{H}_{6} \mathrm{CH}_{3}$ \\
\hline Molecular Weight: & 32.04 & 92.13 \\
\hline Density: & 0.81 & 0.88 \\
\hline Viscosity: & 0.600 & 0.5980 \\
\hline Refractive Index: & 1.3290 & 1.4890 \\
\hline
\end{tabular}


(1960) and Collins (1976).

\subsubsection{Measurements of Porosity, Formation Factor, and Permeability}

\subsubsection{Porosity Measurement}

Since the coating material affects the measurement of resistivity, the core samples were first saturated with brine and resistivity was measured prior to coating. A vacuum saturation technique was used in this process. When the samples were completely saturated with brine, the weights of the wet samples were recorded. Then the pore volume and porosity of a sample were calculated by the following equations:

$$
P V=\frac{W_{w}-W_{d}}{\rho_{w}}
$$

and

$$
\phi=\frac{P V}{B V}=\frac{P V}{\left[\frac{\pi}{4} d^{2} L\right]}
$$

where:

$W_{w}=$ weight of the saturated sample,

$W_{d}=$ weight of the dry sample,

$\rho_{w}=$ density of brine, and

$\mathrm{BV}=$ bulk volume of the sample.

\subsubsection{Formation Factor and Tortuosity Measurement}

A conductivity bridge apparatus (Figure 5.2) was used for measuring (a) resistivity of brine solution (using a dip cell) and (b) resistivity of core sample (using a core holder). The electrolytic dip cell is designed for convenient usage by simply placing the dip cell in a beaker containing the solution in question. The cell with a constant 0.001 was used for this study. The core holder had a 4-electrode system. Because of the limitation of the conductivity unit, only two electrodes were used. According to Rust (1952), both two-electrode and four-electrode methods can yield equally reliable resistivity values.

Sample resistance was read from the conductivity measurement unit. Sample resistivity was then calculated by the following equation: 


\section{4}

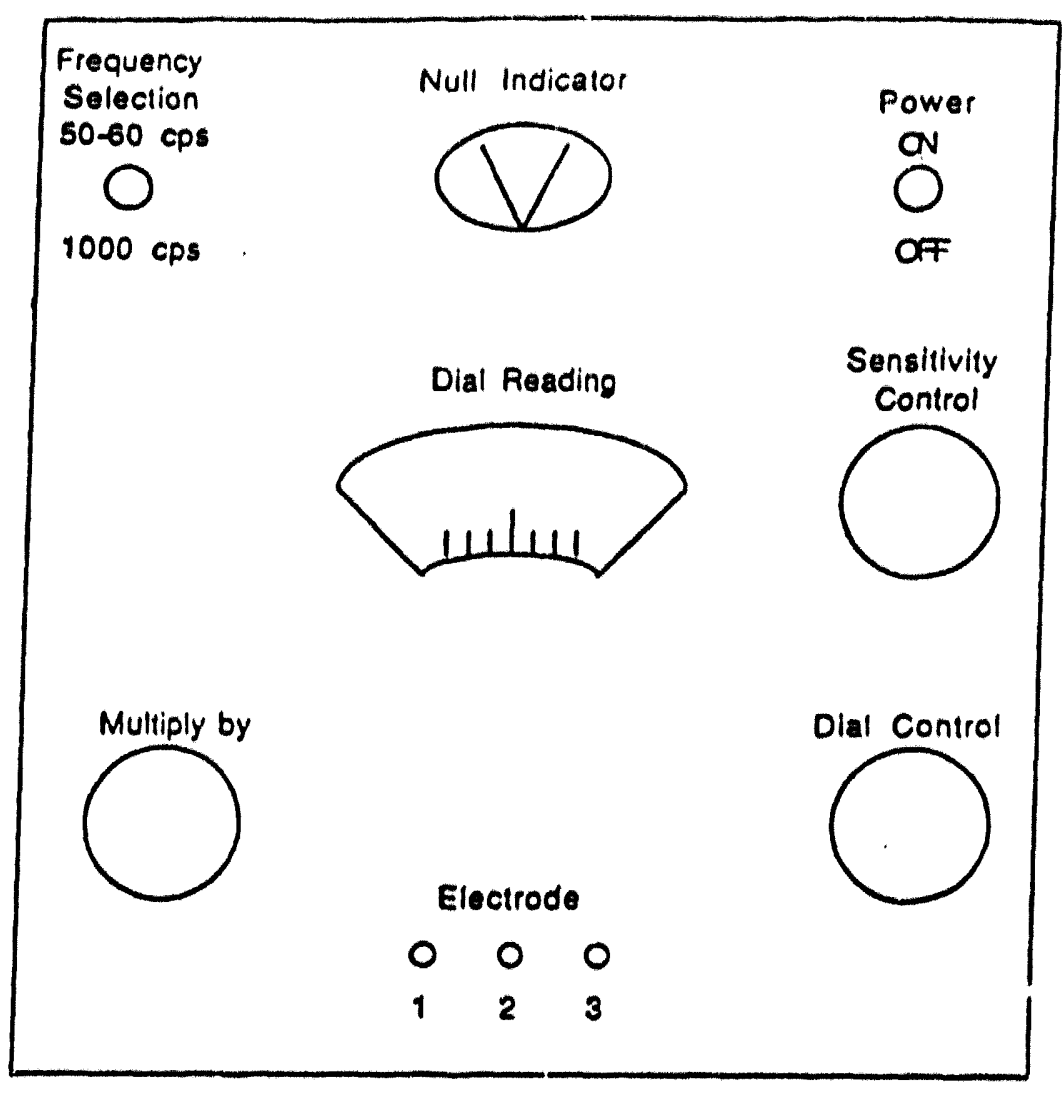

(a) Conirol Panel

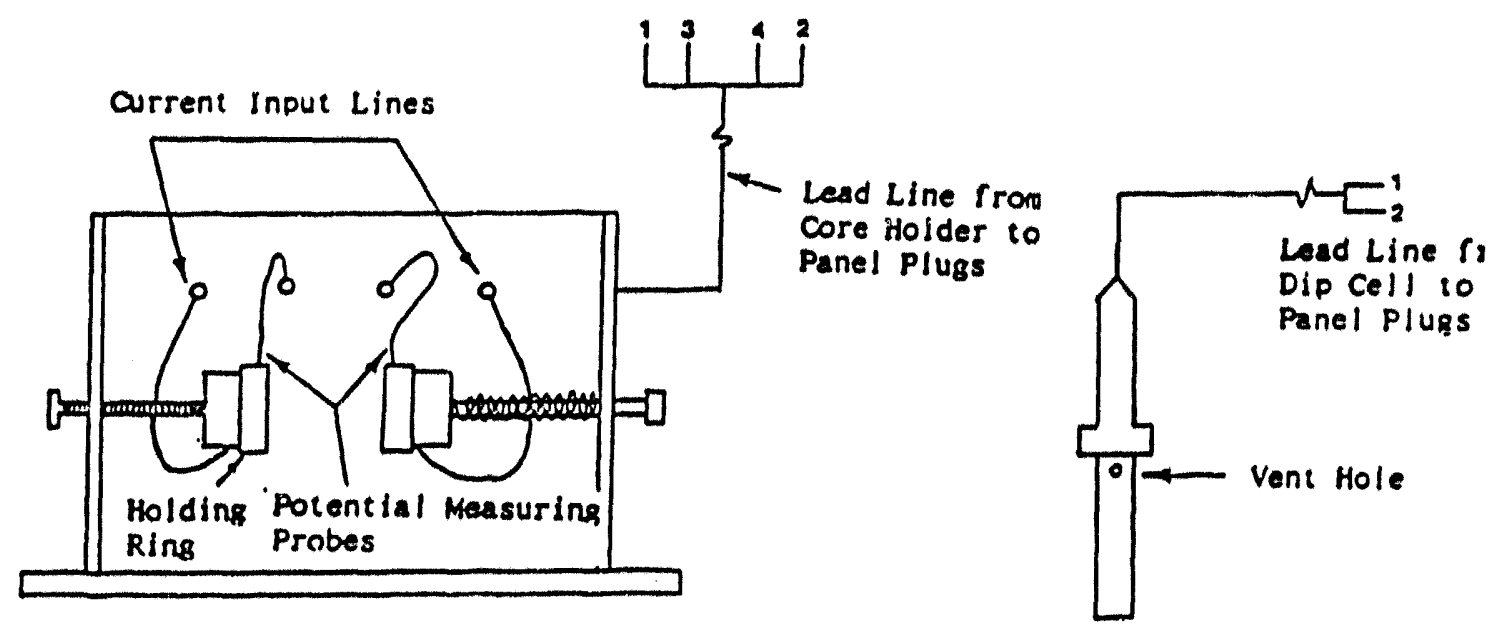

(b) Core Holder

(c) Dip cell

Figure 5.2 : Conductivity bridge apparatus for measuring resistivities 


$$
R_{c}=\left(\frac{A}{L}\right) r_{c}
$$

where $r_{0}$ is a resistance of the sample and $R_{0}$ is a resistivity of the sample.

The formation factor and tortuosity of the sample were further calculated by equations discussed in chapter 3.

\subsubsection{Preparation for Fluld Flow Tests}

After the porosity and resistivity were measured, the core samples were placed back in the Soxhlet solvent extractor for cleaning, and then placid in the oven for drying again. The core was then weighed to determine if the dry weight was consistent with the originally measured value. If the weight was in excess of the original weight, there were some residual fluids and an additional period of drying was needed. After cleaning and drying, the core samples were coated with "J-B" Wold to get ready for permeability and dispersion measurements.

Prior to the beginning of the fluid flow tests, there were two more things to do with the assembled medium. First the sealing of the assembly was checked under certain pressure. The system was tested until no leak was observed.

Second, the gas permeability was measured. This measurement ensured the reliability of the liquid permeability measurements.

\subsubsection{Fluid Permeability Measurement}

Since both processes, permeability measurement and miscible displacement, involve fluid saturation, the resident fluid for miscible displacement was used as fluid for permeability measurement. The procedure was to pull vacuum on the assembled core sample. After about four hours, the fluid - methanol --- was injected into the core with a positive displacement pump. More than three pore volumes of fluid were usually injected to ensure a $100 \%$ saturation. Until the injection pressure stabilized, the liquid permeability was measured by injecting additional fluid and recording the time, pressure, and volume of effluent. It is a general rule that at least two values should be determined at different pressures (or flow rates) to develop a firm value for liquid permeability. Excellent agreement between gas permeability (corrected for "slippage effect") and fluid permeability has been obtained on all core samples.

All the basic rock properties measured in the laboratory are presented in Table 5.4. 
Table 5.4: Measured Petrophysical Properties of Core Samples

\begin{tabular}{||c||c|c|c||}
\hline $\begin{array}{c}\text { Core } \\
\text { Number }\end{array}$ & $\begin{array}{c}\text { Porosity } \\
\text { (8) }\end{array}$ & $\begin{array}{c}\text { Formation } \\
\text { Factor, F }\end{array}$ & $\begin{array}{c}\text { Permeability } \\
\text { K (md) }\end{array}$ \\
\hline B\#1 & 18.8 & 13.32 & 167 \\
\hline B\#2 & 19.7 & 12.99 & 150 \\
\hline B\#3 & 19.1 & 13.40 & 160 \\
\hline B\#4 & 19.2 & 12.77 & 170 \\
\hline B\#5 & 18.8 & 13.47 & 165 \\
\hline B\#6 & 18.7 & 13.49 & 150 \\
\hline B\#10 & 21.4 & 10.46 & 686 \\
\hline B\#11 & 21.5 & 10.70 & 716 \\
\hline B\#12 & 21.1 & 10.95 & 414 \\
\hline
\end{tabular}


Table 5.4: (Continued)

\begin{tabular}{|c|c|c|c|}
\hline $\begin{array}{c}\text { Core } \\
\text { Number }\end{array}$ & $\begin{array}{c}\text { Porosity } \\
\text { (8) }\end{array}$ & $\begin{array}{c}\text { Formation } \\
\text { Factor, F }\end{array}$ & $\begin{array}{c}\text { Permeability } \\
\text { K (md) }\end{array}$ \\
\hline LBH1 & 19.0 & 18.43 & 64 \\
\hline LB\#2 & 18.8 & 18.47 & 69 \\
\hline LB\#3 & 19.2 & 18.38 & 74 \\
\hline LB\#4 & 18.9 & 18.45 & 64 \\
\hline LB\#5 & 19.1 & 18.14 & 83 \\
\hline LB\#6 & 19.4 & 16.55 & 234 \\
\hline LB\#7 & 19.3 & 17.33 & 220 \\
\hline LB\#8 & 19.8 & 16.21 & 268 \\
\hline BN\#1 & 22.8 & 10.95 & 1,817 \\
\hline BN\#2 & 22.5 & 11.40 & 410 \\
\hline BN\#3 & 22.7 & 11.42 & 2,240 \\
\hline BN\#4 & 22.6 & 11.34 & 1,500 \\
\hline
\end{tabular}




\subsection{Misclble Displacement Tests}

To conduct the dispersion measurements on core samples, a miscible displacement test was designed so that an S-shaped concentration profile could be obtained.

\section{S.S.1 Equipment and System Setup}

For the single-phase miscible displacement tests, two major pieces of equipment required were: (1) a constant-rate positive-displacement pump for fluid injection, and (2) - refractometer for measuring the effluent concentration.

The refractometer was used for determining the effluent concentration. This device was designed based on the optical principle that each fluid has a unique value of refractive index (R.I.). If the refractive indices of two fluids are known, their percentage in a mixture can be determined from the measured refractive index for this mixture.

The system setup is illustrated in Figure 5.3. In this system, the system measurement orror was minimized. This can be reflected from the following design considerations:

(a) Since two different types of fluids were to be used in the experiment, two separate flow systems were established to avoid flushing the flow system after injecting one fluid. If the flushing is not complete, the effluent concentration will be in error.

(b) Because relatively low pressure (less than 5 psi) was applied in the experiment, Manometers, rather than pressure gauges, were used to record pressure in order to secure accurate permeability measurements.

(c) As indicated in the theoretical analysis, a constant flow rate is crucial to dispersion measurement. To obtain a constant flow rate, a constant injection pressure should be maintained during the whole process of displacement. However, due to the compressibility of the fluids used, it takes some time for inlet pressure to build up from initially zero to a constant value. This initial pressure variation causes fluctuations of flow rate inside the core sample. As a result, the dispersion process thus measured can not accurately reflect the property of the porous medium. Therefore, establishing a constant injection pressure prior to beginning of the displacement was necessary.

\subsubsection{Operation Procedure of the Experiment}

Prior to the beginning of the test, the endplate-involved volume was determined. With the core $100 \%$ saturated with the resident fluid, the medium (core sample plus core holder) was removed from the system and weighed. With the previously measured dry 


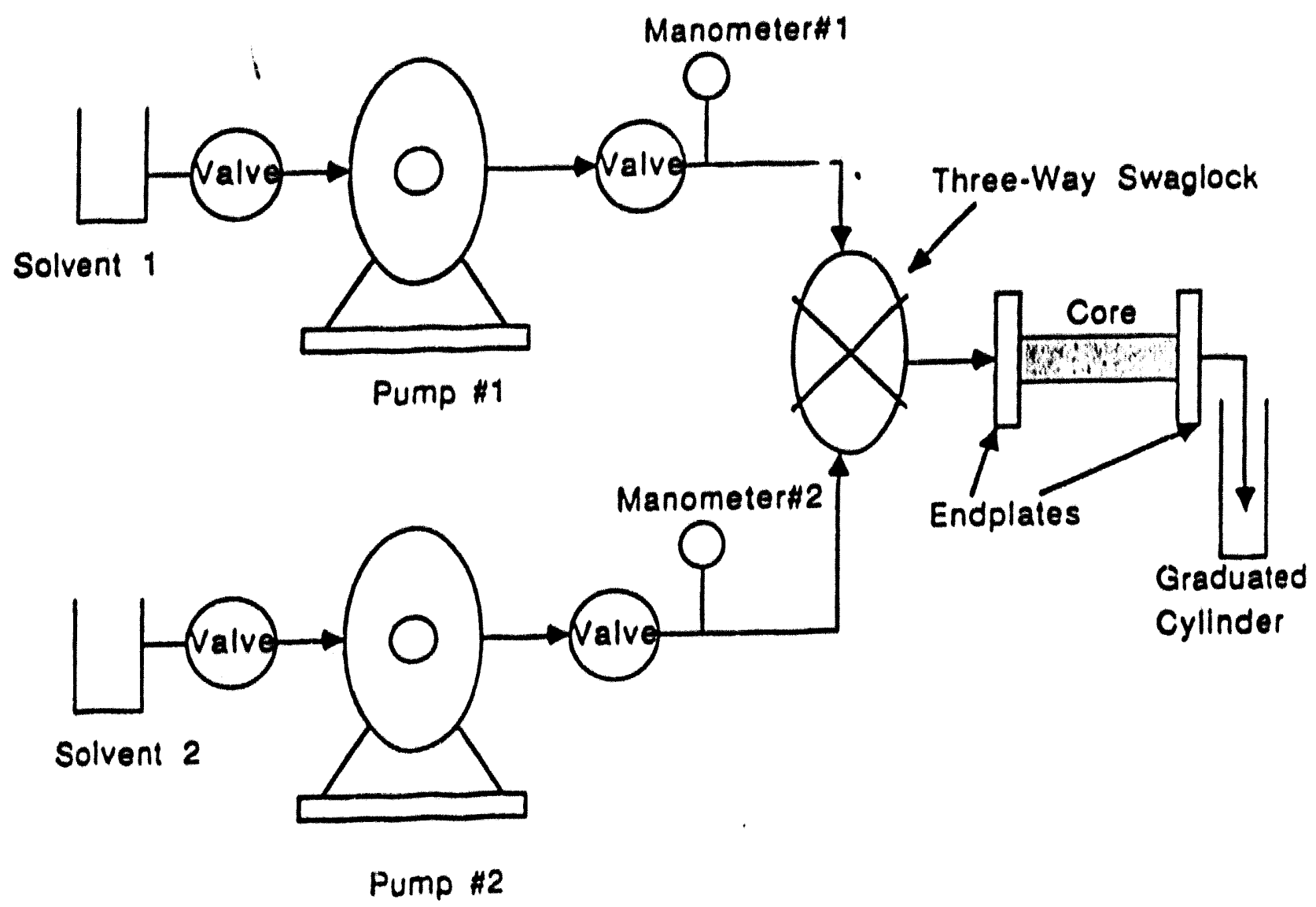

Figure 5.3: System setup for steady-state miscible displacement test 
weight of the medium and the density of the fluid, the total effective pore volume was determined. This total volume minus the known pore volume of the core gave the volume of endplates. The total volume was used in the calculation instead of core pore volume.

Based on the above discussions, the procedure of conducting a rellable miscible displacement can be described below:

(a) With the three-way valve open to the resident fluid injection line,pump the resident fluid (methanol) at the pro-determined flow rate until the pressure drop stablilizes. This step can be done during the permeablitty measurement process;

(b) Close the three-way valve and switch the pump to the displacing fluid (toluene);

(c) Establish a constant injection pressure by pumping the displacing fluid with the three-way valve closed until the same pressure drop as established in step (a) is reached;

(d) Open the three-way valve to the displacing fluid injection line. At the rame time, start the timer;

(c) Record the time when watching the fluid ample collection tubes;

(f) Read the volume of effluent and refractive index for each fluid cample. If this step can not be done immediately, the tubes are capped to prevent vaporization of the fluid sumple; and

(B) Continue steps (e) and (f) until more than 99 percent displacing fluld is recorded in the effluent. 


\section{CHAPTER 6}

\section{CHARACTERIENNG ROCK EETEROGENEITHES WTTH DISPERSION MIRASUREMIENT}

The term "heterogeneity" is quite often a convenient catch-all phase of uncertain meaning, loosely applieo to rock variability in an ambiguous context (Alpay, 1972). Here the term is uned to describe the miscible displacement anomalles or the dynamic aspects of fluid flow in porous media. In this chapter, a new approach to determining the heterogeneity factor from the measured concontration profile will be developed.

\subsection{Measurement of Beterogeneity}

Bven though numerous investigations of reservoir heterogeneity have been made during past decades, how to measure it quantitatively is still an open question. However, ince the class of heterogeneities that is encountered in most cases is at the macroscopic level outlined in Sections IIA, and IBB of Table 6.1, there is a good reason to belleve that the heterogenelty factor and the permeability variation should be related. In addition, permeability is also more sensitive to conductance, mixing, and capillary pressure than porosity and other macroscopic parametors.

By applying the frnetional flow concept ascocinted with immiacible displacement to miscible displacement, Koval (1963) defined the heterogeneity factor in terms of the percentage of solvent recovery at one pore volume injection in a matched viscosity flood. With Koval's approach, a homogeneous systom is abitrarlly defined as one in which the oil recovery at one pore volume solvent injected in a matched viscosity flood is greater than 99 percent. By choosing this high value of recovery at one pore volume injected, only syatems which have no significant dispersion would be termed homogeneous. In addition, the percentage of oll necovery is inconvenient to measure in miscible displacement tests in the laboratory. Instead, the solvent concentration is practically measured at the outlet of the system. It should be more convenient to use the effluent concentration data for ovaluating the mck heterogeneity.

According to the Buckley-Loverett equations (Buckley and Loverett, 1942) and the Koval's discussion, the fractional flow equation for a matched viscosity flood is: 
Table 6.1: Elements of Heterogeneity That Influence

Fluid Flow in a Reservoir (after Alpay, 1972)

\begin{tabular}{|c|c|c|}
\hline $\begin{array}{c}\text { I. } \\
\text { Microscopic } \\
\text { Heterogeneity }\end{array}$ & $\begin{array}{l}\text { II. } \\
\text { Macroscopic } \\
\text { Heterogeneity }\end{array}$ & $\begin{array}{l}\text { III. } \\
\text { Megascopic } \\
\text { Heterogeneity }\end{array}$ \\
\hline $\begin{array}{l}\text { Pore-to-poral } \\
1.6 .1\end{array}$ & $\begin{array}{l}\text { Well-to-well or } \\
\text { intra-format lonal, } \\
\text { l.e. }\end{array}$ & $\begin{array}{l}\text { Formational, elthar } \\
\text { leldwlde/reglonal, } \\
\text { l.e., }\end{array}$ \\
\hline $\begin{array}{l}\text { A. Pore-size dle- } \\
\text { tribution }\end{array}$ & $\begin{array}{l}\text { A. Stratifleation } \\
\text { characteriaticas }\end{array}$ & A. Reservolr geometry \\
\hline B. Pore geometry & $\begin{array}{l}\text { (1) Nonundform } \\
\text { otratifleation }\end{array}$ & $\begin{array}{l}\text { (1) over-ail otruo- } \\
\text { tural framework, } \\
\text { faults, dipping } \\
\text { etrata, etc. }\end{array}$ \\
\hline \multirow[t]{7}{*}{$\begin{array}{l}\text { c. Dead-end pore } \\
\text { epace }\end{array}$} & $\begin{array}{l}\text { (2) Stratification } \\
\text { contrauts }\end{array}$ & $\begin{array}{l}\text { (2) over-ald otrat 1- } \\
\text { graphle irame- } \\
\text { work, bar,blanket, } \\
\text { channel illd, etc. }\end{array}$ \\
\hline & $\begin{array}{l}\text { (3) stratifleation } \\
\text { continuity }\end{array}$ & $\begin{array}{l}\text { B. Hyperpermeablilty } \\
\text { oriented natural } \\
\text { eracture oystems }\end{array}$ \\
\hline & $\begin{array}{l}\text { (4) Insulation to } \\
\text { crosetlow }\end{array}$ & \\
\hline & $\begin{array}{l}\text { B. Permeablilty } \\
\text { characteristics: }\end{array}$ & \\
\hline & $\begin{array}{l}\text { (1) Nonuniform } \\
\text { permeability }\end{array}$ & \\
\hline & $\begin{array}{l}\text { (2) Permeability } \\
\text { trends }\end{array}$ & \\
\hline & $\begin{array}{l}\text { (3) Directional } \\
\text { permeablility }\end{array}$ & \\
\hline
\end{tabular}




$$
f_{s}=\frac{H S_{s}}{1+S_{s}(H-1)}
$$

where:

$$
\text { H = a heterogeneity factor used by Koval, }
$$

$f_{1}=$ flow fraction of solvent of effluent concentration, and

$S_{\mathbf{s}}=$ solvent saturation inside the porous medium.

Since the driving proxess is similar to that of Koval, the heterogeneity factor is essentially the same. The difference is the way it is determined. Therefore, the same notation for the heterogeneity factor, $\mathrm{H}$, is used.

Using material balance, the frontal equation is further derived as

$$
\frac{d f_{s}}{d S_{s}}=\frac{1}{I}
$$

Substituting Eq.(6.2) into Eq.(6.1) yields

$$
f_{s}=\frac{H-\sqrt{\frac{H}{I}}}{H-1}
$$

At one pore volume injection, i.e., $\mathrm{I}=1$, Eq.(6.3) becomes

$$
f_{s}=\frac{H-\sqrt{H}}{H-1}
$$

Solving for $H$ in the above equation and substituting $f_{1}$ with $C_{\text {OI=1 }}$, the evaluation equation for rock heterogeneities in terms of concentration at one pore volume of injection is obtained:

$$
H=\left[\frac{f_{s}}{1-f_{s}}\right]^{2}
$$

The plot of Eq.(6.5) is shown in Figure 6.1.

From Figure 6.1, it can be seen that as the core sample becomes more homogeneous (i.e, the heterogeneity factor is approaching 1), the solvent cut at one pore volume injection approaches 0.5 . This explains why the concentration at one pore volume injection is often greater than 50 percent in consolizated sandstone samples. Eq.(6.5) 


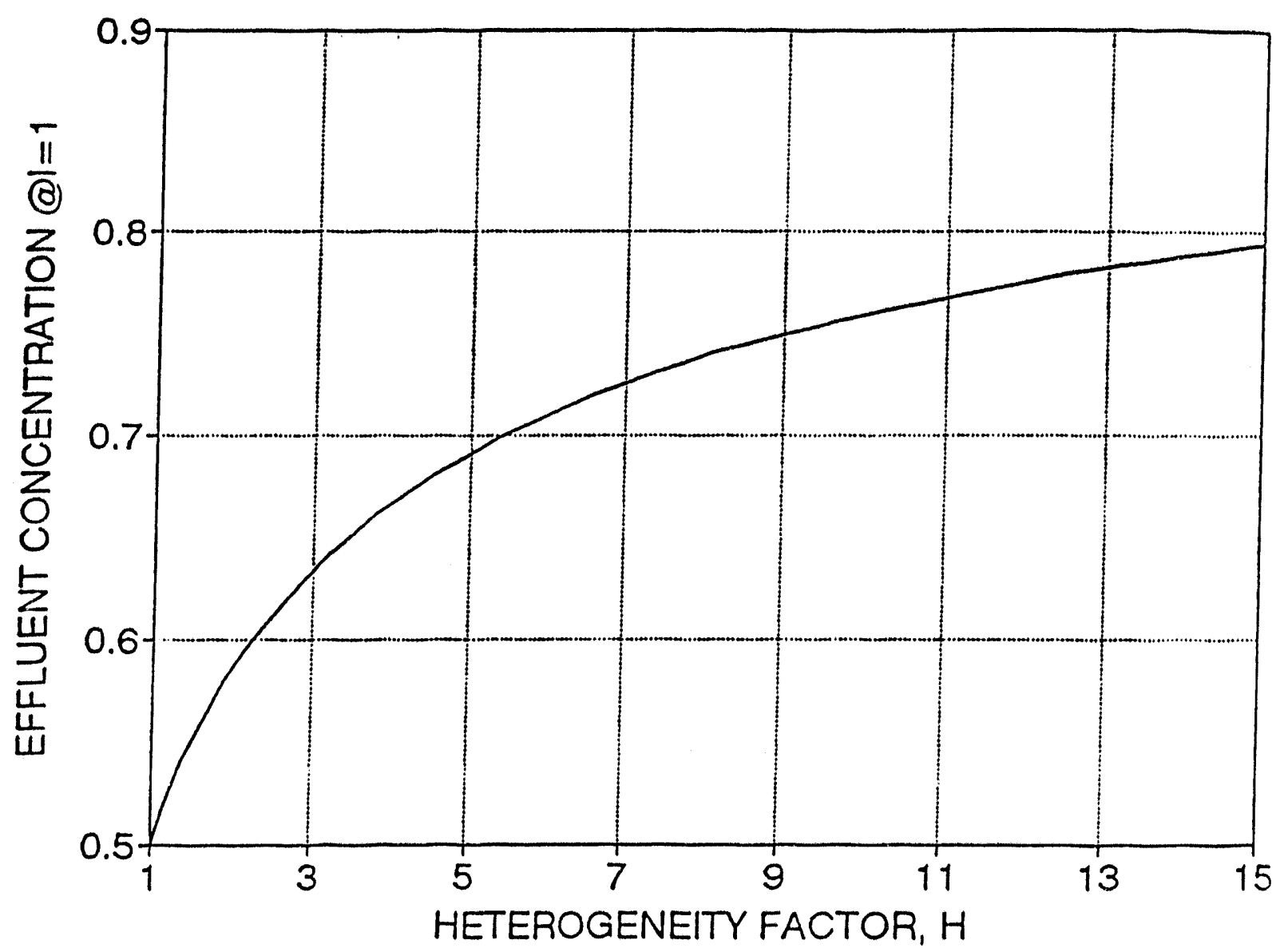

Figure 6.1: Defining heterogeneity factor in terms of solvent cut at one pore volume of solvent injection in a matched viscosity flood 
also means that the greater the deviation of the concentration at one pore volume injection from 50 percent, the more heterogeneous the porous medium.

All the core samples described in the last chapter have been evaluated using Eq.(6.5) for their heterogeneity factors and the results are presented in Table 6.2.

\subsection{Correlations of Heterogeneity Factor with Rock Parameters}

\subsubsection{Heterogeneity Factor Versus Permeability}

Apparently permeability should be a function of rock heterogeneity. This relationship has been presented in Figures 6.2 through 6.5. However, this observation may not be consistent because permeability is related to the magnitude of pore sizes, while heterogeneity is related to the distribution of pore sizes.

\subsubsection{Heterogeneity Factor versus Tortuosity}

In general, tortuosity should increase as the heterogeneity factor increases. For each group of core samples, this relationship is shown in Figure 6.6. 
Table 6.2: Evaluated Heterogeneity Factors of Core Samples

\begin{tabular}{|c|c|c|}
\hline Core No. & $C_{\text {Q1=1 }}$ & $H$ \\
\hline$B \# 1$ & 0.54 & 1.378 \\
\hline$B \# 2$ & 0.52 & 1.174 \\
\hline$B \# 3$ & 0.53 & 1.272 \\
\hline$B \# 4$ & 0.54 & 1.378 \\
\hline$B \# 5$ & 0.53 & 1.272 \\
\hline$B \# 6$ & 0.54 & 1.378 \\
\hline$B \# 10$ & 0.625 & 2.662 \\
\hline$B \# 11$ & 0.63 & 2.899 \\
\hline$B \# 12$ & 0.63 & 2.899 \\
\hline$L B \# 1$ & 0.635 & 3.03 \\
\hline$L B \# 2$ & 0.63 & 2.90 \\
\hline$L B \# 3$ & 0.63 & 2.90 \\
\hline$L B \# 4$ & 0.635 & 3.03 \\
\hline$L B \# 5$ & 0.63 & 2.90 \\
\hline
\end{tabular}


Table 6.2: (Continued)

\begin{tabular}{|c|c|c|}
\hline Core No. & $C_{\text {GI } 1}$ & $H$ \\
\hline LB\#6 & 0.605 & 2.35 \\
\hline LB\#7 & 0.60 & 2.25 \\
\hline LB\#8 & 0.655 & 3.60 \\
\hline BN\#1 & 0.61 & 2.45 \\
\hline BN\#2 & 0.63 & 2.90 \\
\hline BN\#3 & 0.60 & 2.25 \\
\hline$B N \# 4$ & 0.615 & 2.55 \\
\hline
\end{tabular}




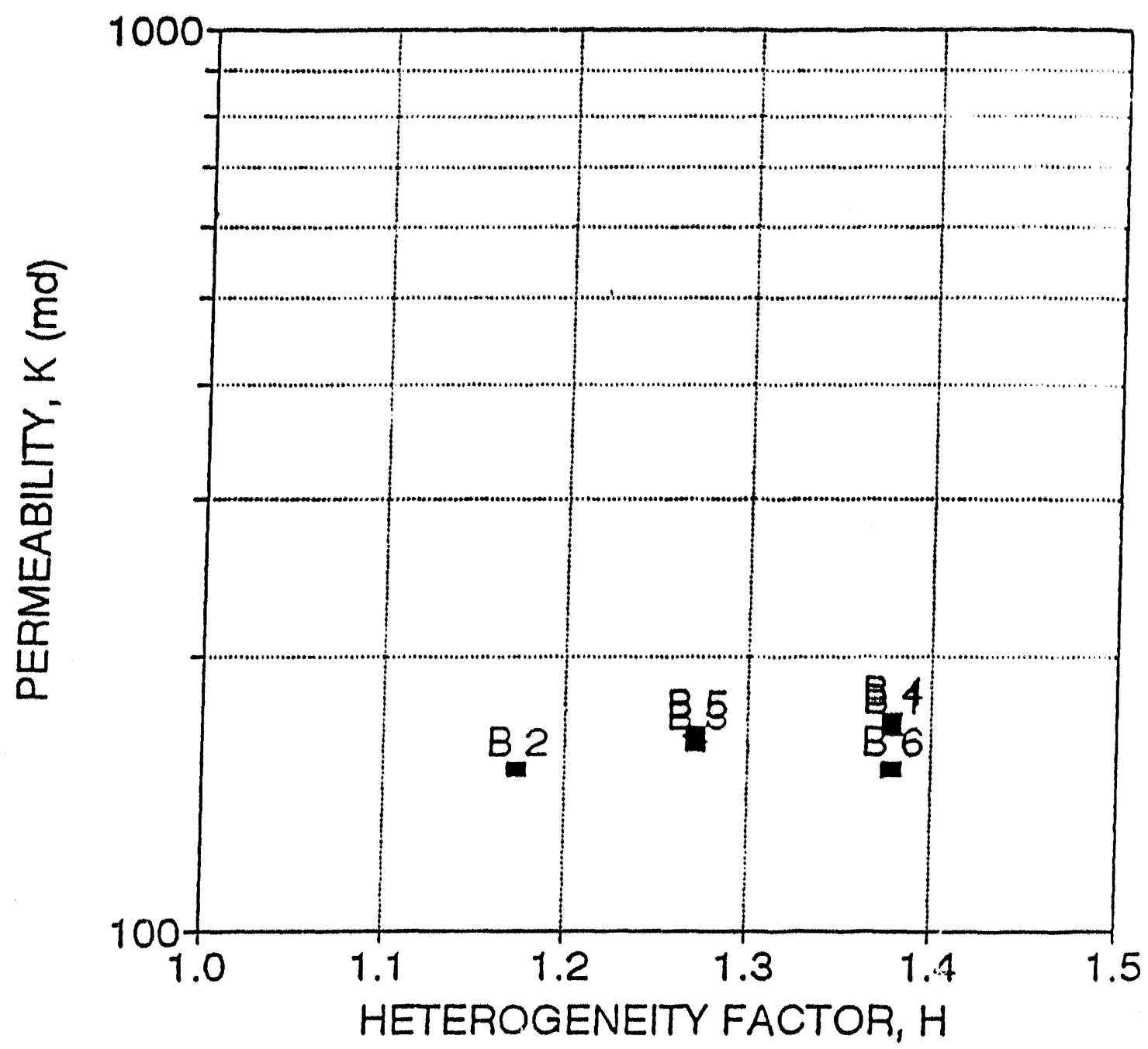

Figure 6.2: Permeability versus heterogeneity for Group \#1 Berea sandstone samples 


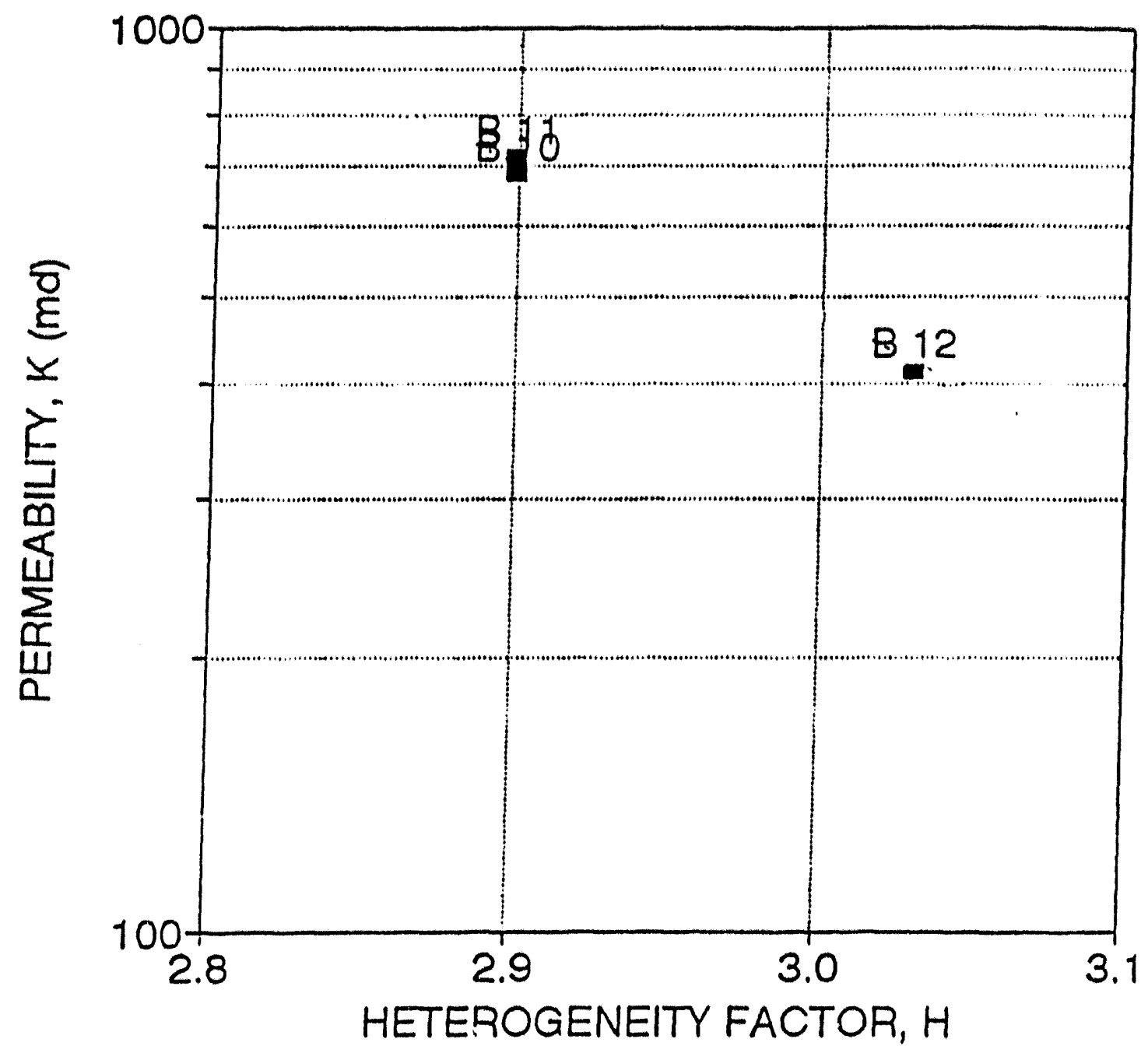

Figure 6.3: Permeability versus heterogeneity for Group \#2 Berea sandstone samples 


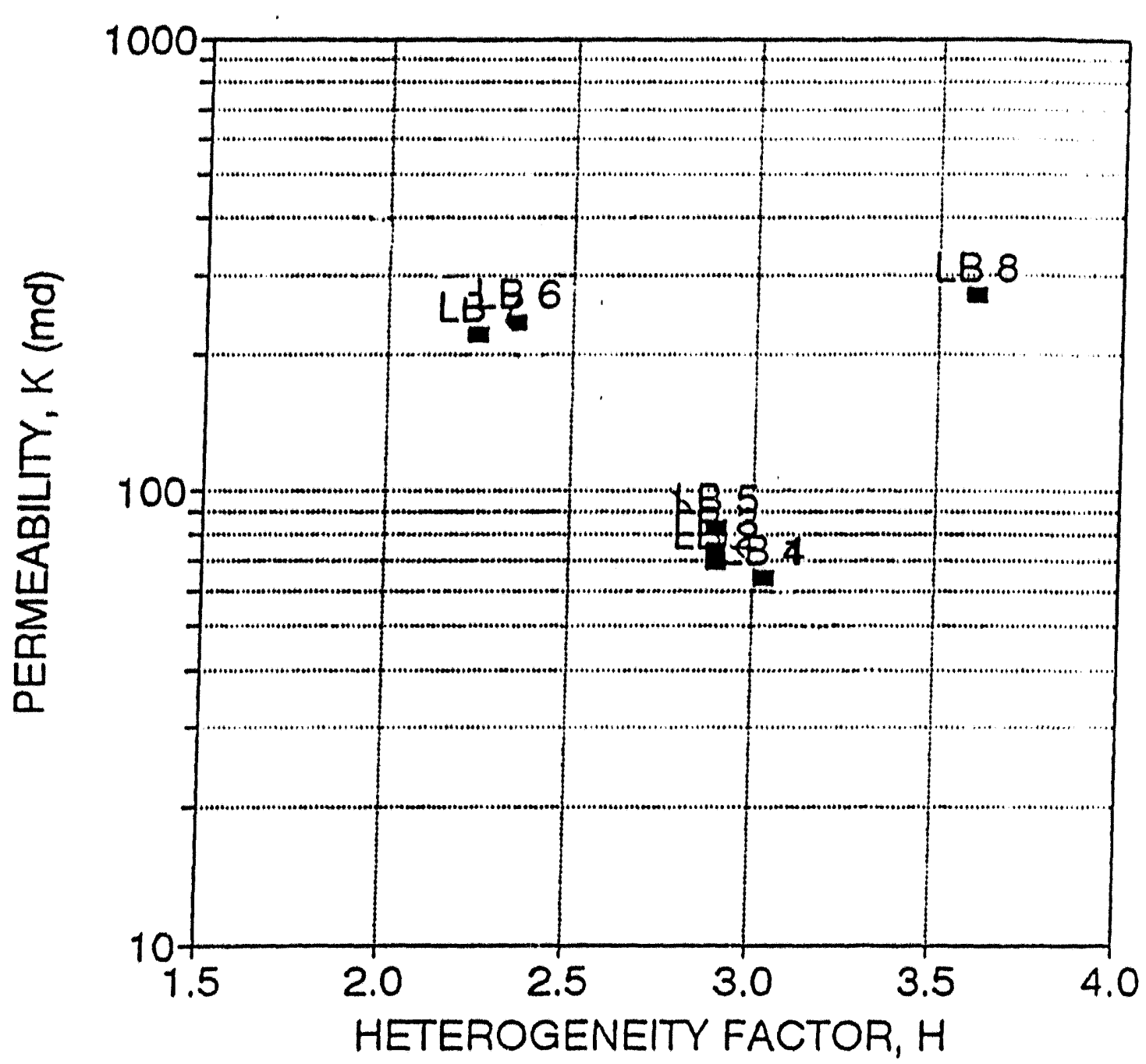

Figure 6.4: Permeability versus heterogeneity factor for $1.5^{\prime \prime}$ diameter Berea sandstone samples (Group \#3 and $\$ 4$ ) 


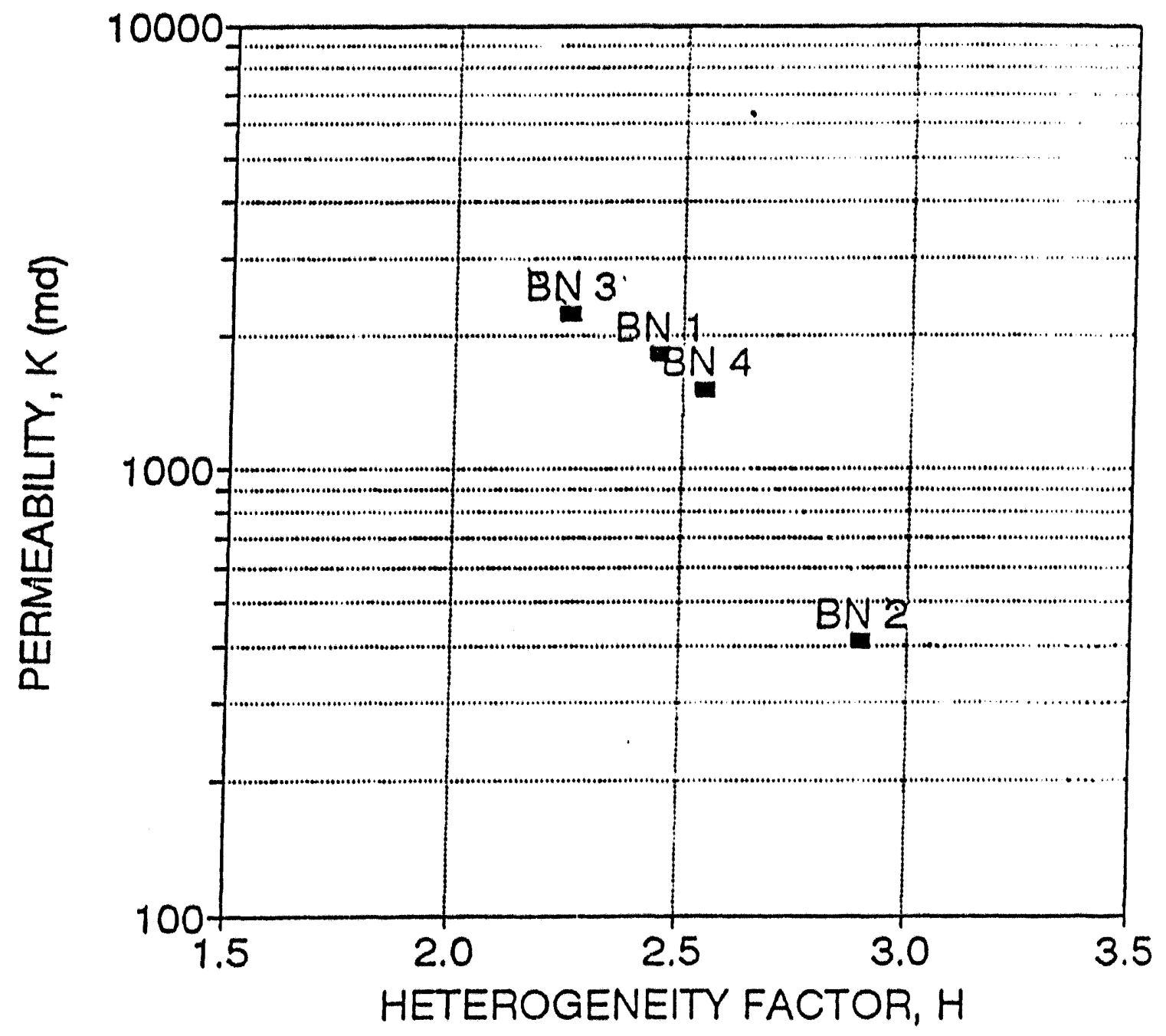

Figure 6.5: Permeability versus heterogeneity for 1 " diameter Brown sandstone samples (Group \#5) 


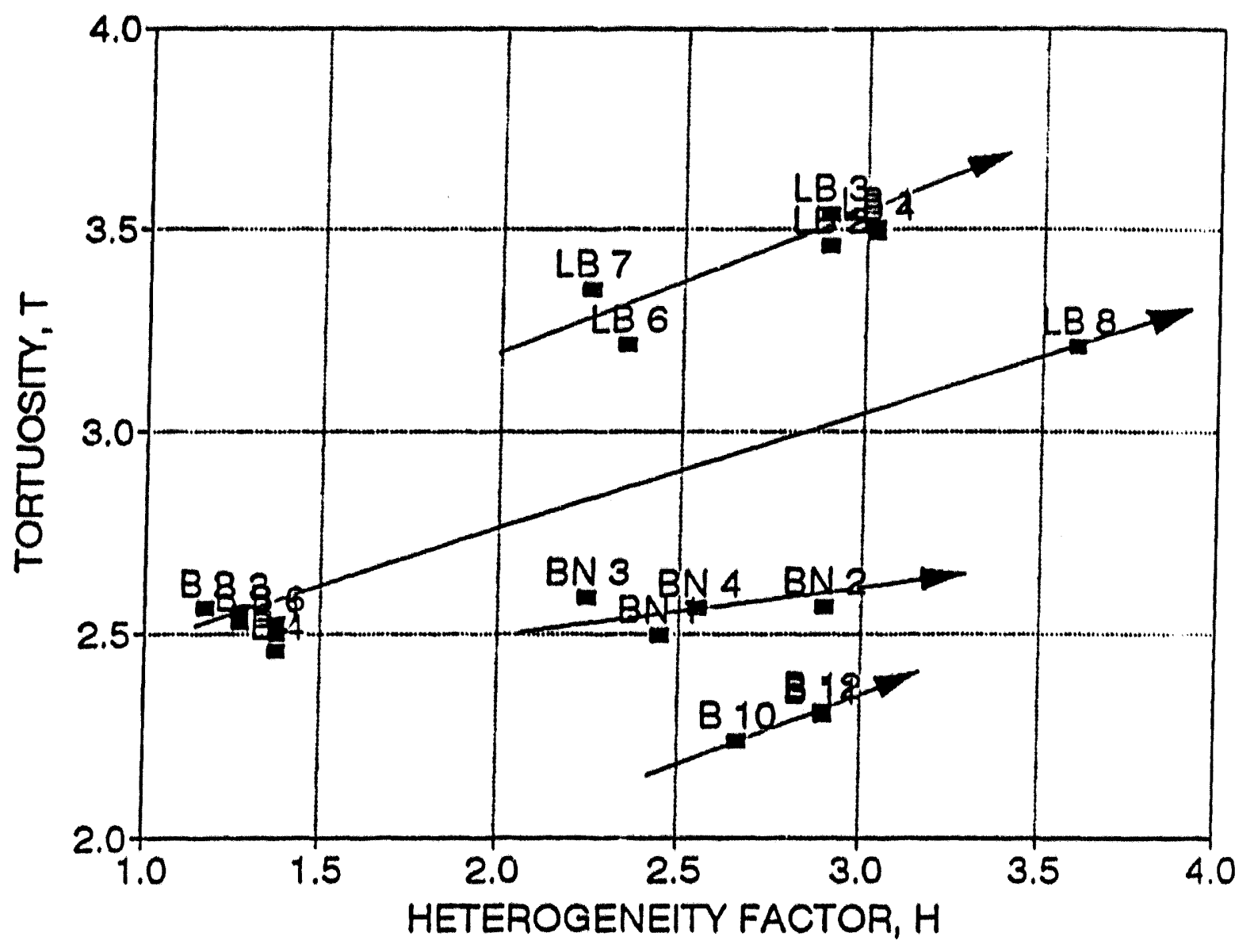

Figure 6.6: Heterogeneity factor as a function of tortuosity in Berea and Brown sandstone samples 


\section{3}

\section{CHAPTER 7}

\section{CHARACTERIZNNG RESERVOIR ROCKS WITH DISPERSTVITY}

In chapter 6, the rock heterogeneities have been evaluated from the concentration profiles. In this section, a "standard dispersivity parameter" will be determined from the concentration profile for characterizing the rock.

\subsection{Dispersion Model for Matching Concentration Proflles}

To calculate dispersivity from a concentration profile, an appropriate dispersion model must be applied. By matching the concentration profile with the dispersion model, a dispersivity number for that rock can be calculated. For a symmetrical S-shaped concentration profile the convection-dispersion model yields the dispersivity number which is simply the dispersion coefficient divided by the fluid velocity. For an asymmetrical S-shaped concentration profile, the convection-dispersion model fails. A more complex model such as the dispersion-capacitance model should be used in these cases. The concentration profiles measured on the sandstone core ramples have indicated the asymmetrical S-shaped curves by having the long tails. For these concentration profiles, the Coats-Smith capacitance model seems to be appropriate.

However, three fitting parameters in the dispersion capacitance model cause difficulty in determining one unique dispersivity number because of the multiple solutions. Traditionally in the literature, this model was used to match concentration profiles by arbitrarily assigning a value to one parameter and adjusting the remaining two parameters. Multiple solutions are usually obtained. Also none of the solutions are physically meaningful. To overcome the deficiency of the model, the following efforts have been made by redefining the flow fraction and its physical meaning, and using an equivalent dispersivity parameter to represent the concentration profile.

\subsubsection{New Definition for the parameter $f$ in the Dispersion Capacitance Model}

In the dispersion capacitance model, parameter $f$ is conceptually defined by Coats and Smith (1964) as the flow fraction that contributes to the dynamic fluid flow during miscible displacements. Unfortunately, this definition has not given any explanation for its existence and its physical meaning because it has never been directly tested in the laboratory. An alternative approach to explain the parameter $f$ is to redefine the parameter $f$ based on its physical meaning. 
Since the solution equation of the dispersion capacitance model is very complex, the derivation of the mathematical forms of its first and second derivatives to pore volume is very difficult. Through mathematical analysis, the following conclusion can be obtained:

$$
\left[\frac{\partial C}{\partial\left(V_{1} / V_{p}\right)}\right]_{O\left(V_{1}, V_{1}\right) v} \stackrel{\rightarrow \text { maximum }}{ }
$$

This equation says that the first derivative of concentration reaches a maximum at $V / V$, equals $f$. Therefore, $f$ is numerically equal to the pore volume fraction which corresponds to the peak of the first derivative concentration curve, as illustrated in Figure 7.1.

The peak of the first derivative concentration curve means the maximum miscible displacement efficiency. The corresponding pore volume fraction, $f$, can be referred to as the dispersion fraction (or mixing frnction). To differentiate this definition with the term of flow fraction, hereafter this parameter $f$ will be replaced by $f_{d}$.

The definition of the parameter $f_{d}$ gives its physical meaning as the maximum pore volume fraction that contributes to the dynamic miscible displacement process by mechanical dispersion. The rest of the pore volume $\left(1-f_{0}\right)$ is the stagnant pore spaces which contribute to the miscible displacement only by molecular diffusion. Therefore, the parameter, $1-f_{d}$ can then be named the diffusion fraction. This essentially divides the concentration curve into two parts: the left-part and the right-side part by drawing a vertical line through the disporsion fraction point as illustrated in Figure 7.1. The left part can be called the front dispersion which is dominated by the mechanical dispersion mechanism; and the right-side part can be called the rear dispersion which is dominated by the diffusion mechanism. The definitions for these terms are also closely tied with the dispersion capacitance model where the dispersion coefficient $D_{L}$ controls the front dispersion and the mass transfer coefficient $M$ controls the rear dispersion.

By definition, the parameter of dispersion fraction $f_{d}$ can be directly determined from the first derivative concentration curve. Once this parameter is well defined, a unique solution to the capacitance-dispersion can be obtained. Further discussion can be found in the section 7.2.2 where equivalent dispersivity is defined.

\subsubsection{Relationship Between Dispersion Fraction and Heterogeneity Factor}

By drawing a vertical line at 1 pore volume $\left(V_{i} / V_{p}=1\right)$, the whole concentration curve is divided into two parts. According to the material balance, the area below the curve on the left side of the vertical line must be ozual to the area above the cu.ve on 


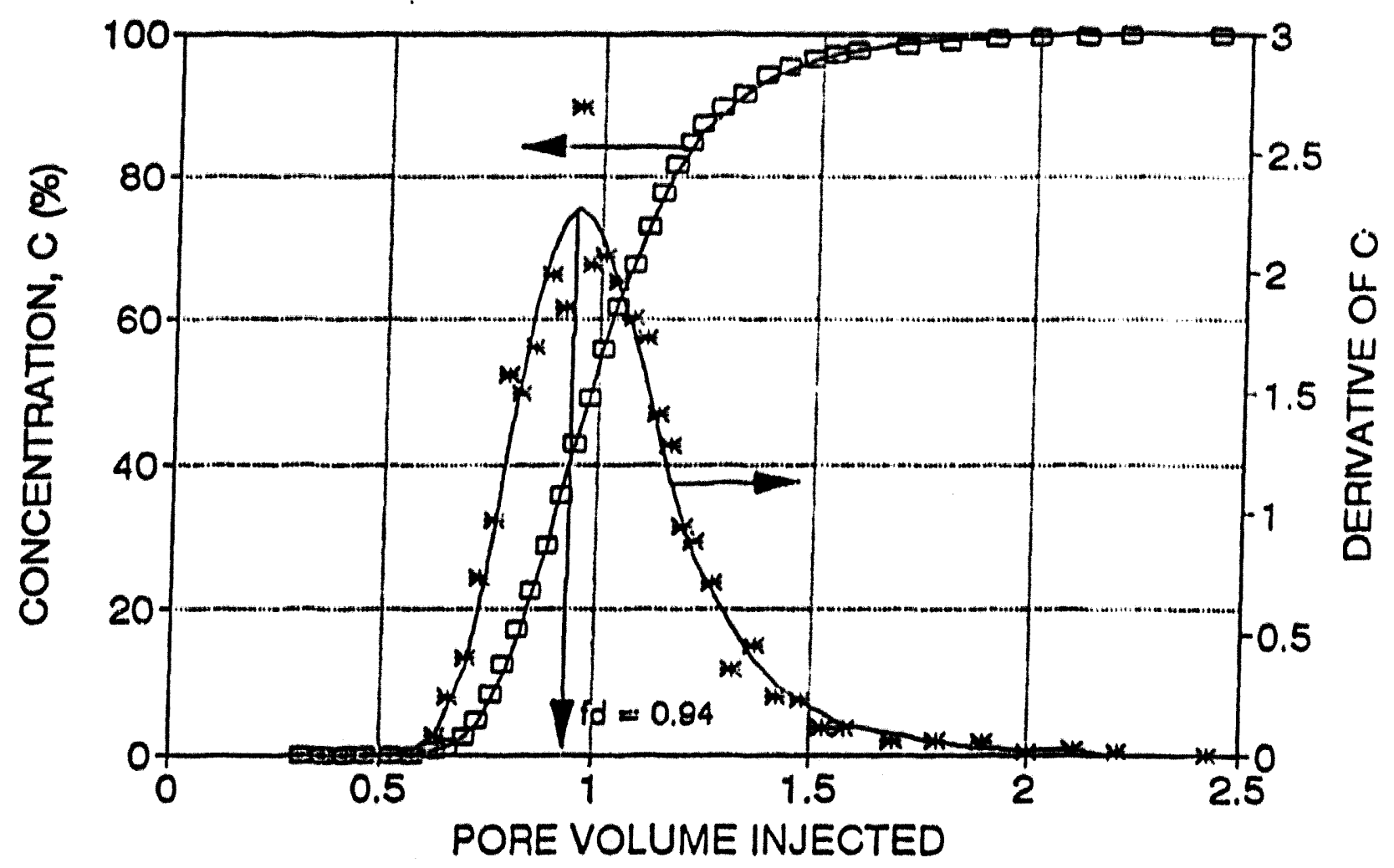

D EXP. - MODEL $*$ EXP. $\longrightarrow$ MODEL

Figure 7.1: Defining dispersion fraction based on the peak of the first derivative of concentration 
the right side of the vertical line. Due to the long tall on the concentration curve which is caused by the heterogeneity of the medium, the concentration at one pore volume injection must be greater than 50 percent. Accordingly, the peak of the concentration derivative curve must fall on the left side of the vertical line, which results in the dispersion fraction less than 1 . Now if the concentration at 1 pore volume injection is designated as $\left.\mathrm{C}\right|_{e, 1=1}$ and the pore volume corresponding to the peak of the concentration derivative curve as II emar, their relationship can be obtained from the solution to the convection-dispersion model:

$$
\left.C\right|_{01-1}=\frac{1}{2}+\sqrt{\frac{1-\left.I\right|_{0 \text { mat }} ^{2}}{\left.8 \pi I\right|_{\text {pomit }}}}
$$

This equation says that $\mathrm{C} \mid$ oim increases as I | opuk decreases. The variation of I | oput ranges from 0.0 to 0.1 , and accordingly $\left.\mathrm{C}\right|_{\text {ot }-1}$ ranges from 1.0 to 0.5 . For a homogeneous medium, $\left.I\right|_{\text {epout }}=1.0$ and $\left.C\right|_{\text {or }=1}=0.5$.

A similar relationship to Bq. (7.2) can aleo be dertved from the solution to the dispersion capacitance model. Since this solution is complex, it is difficult to obtain an explicit analytical equation. For the sake of simplicity, Bq.(7.2) will be applied below to discuss the relationship between the dispersion fraction and the heterogeneity factor.

As discussed in a previous chapter, $\mathrm{C} \mid$ oim is related to rock heterogeneity. Bq. (7.1) indicates that PV | one is related to the dispersion fraction. Substituting Eq.(7.2) into Bq. (6.5) yields

$$
H=\left(\frac{\frac{1}{2}+\sqrt{\frac{1-f_{d}^{2}}{8 \pi f_{d}}}}{\left.\frac{1}{2}-\sqrt{\frac{1-f_{d}^{2}}{8 \pi f_{d}}}\right)^{2}}\right.
$$

where I | oport is replaced by dispersion fraction $f_{d}$ and $C \mid$ and by effluent concentration of solvent in flux. By plotting Eq.(7.3) in Figure 7.2, it can be scen that the dispersion fraction decreases as the heterogeneity factor increases.

The relationship indicated by Bq.(7.3) is also supported by the laboratory data (Figures 7.3 and 7.4). The rock heterogeneity is a function of the pore size distribution. A higher heterogeneity factor means wider pore size distribution, which then results in a larger stagnant pore volume and a smaller dispersion fraction. 


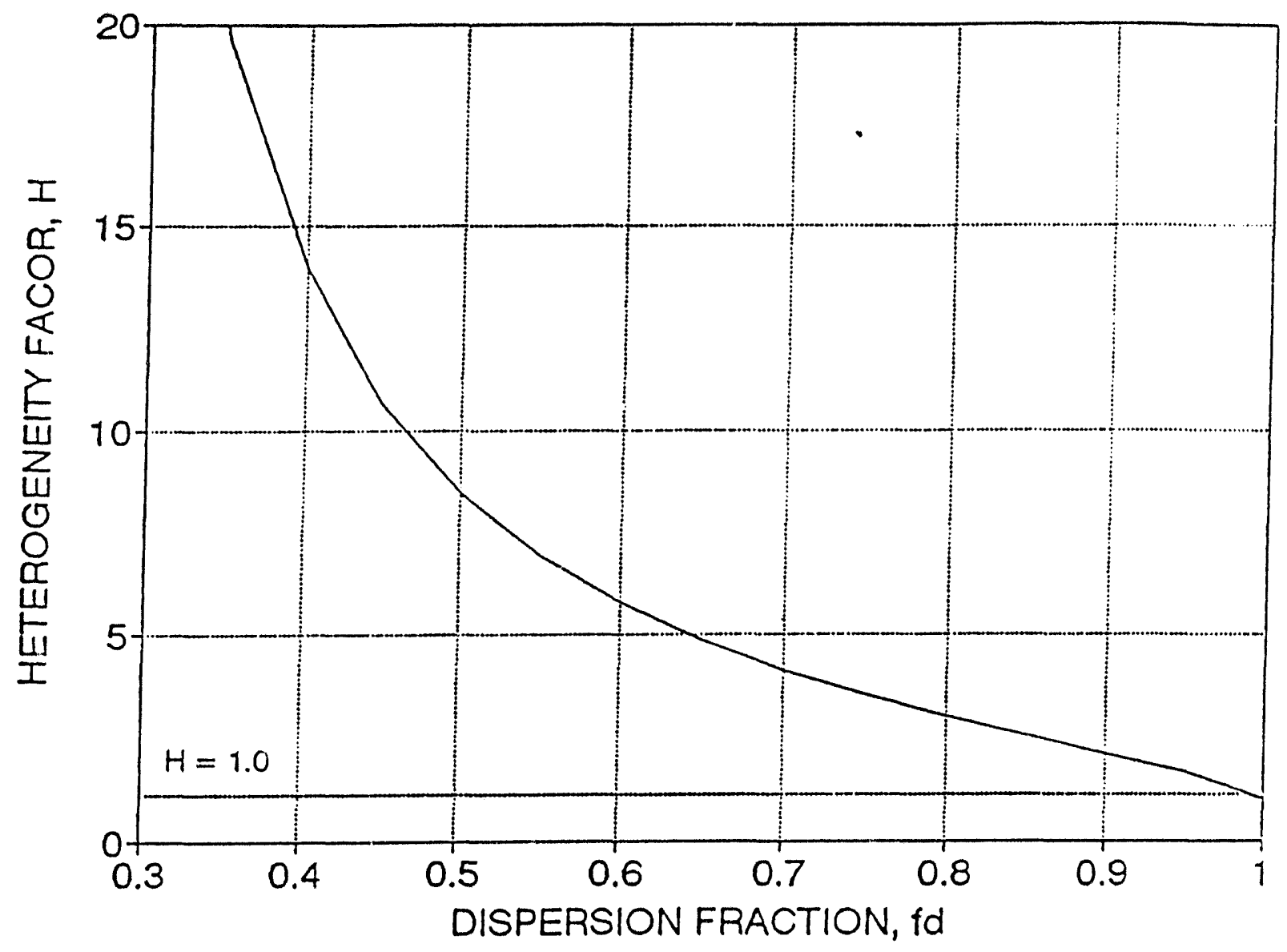

Figure 7.2: Relationship between dispersion fraction $\left(f_{d}\right)$ and heterogeneity factor $(\mathrm{H})$ 


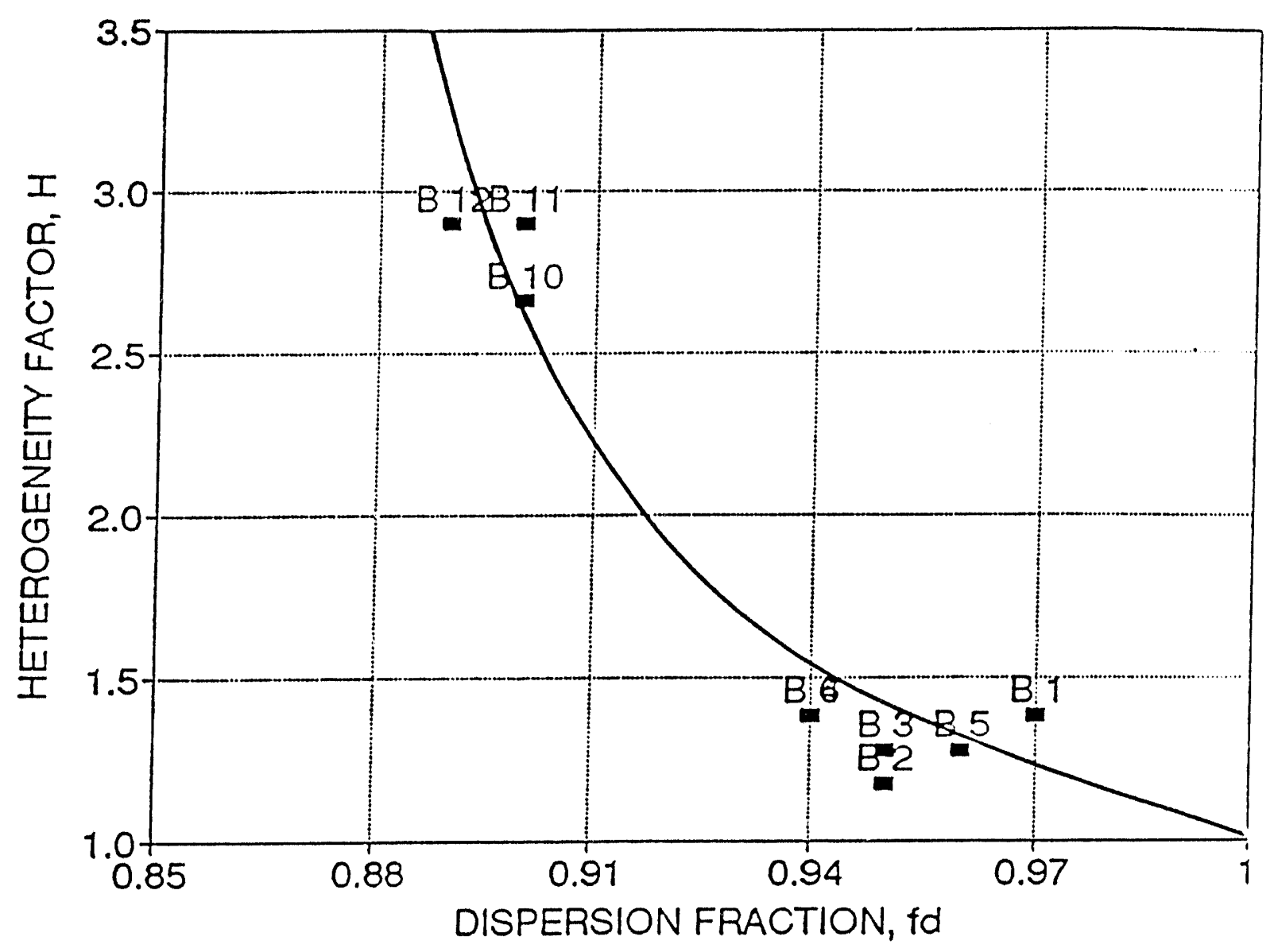

Figure 7.3: Dispersion fraction as a function of heterogeneity factor in $1^{\text {" diameter Berea Samples }}$ 


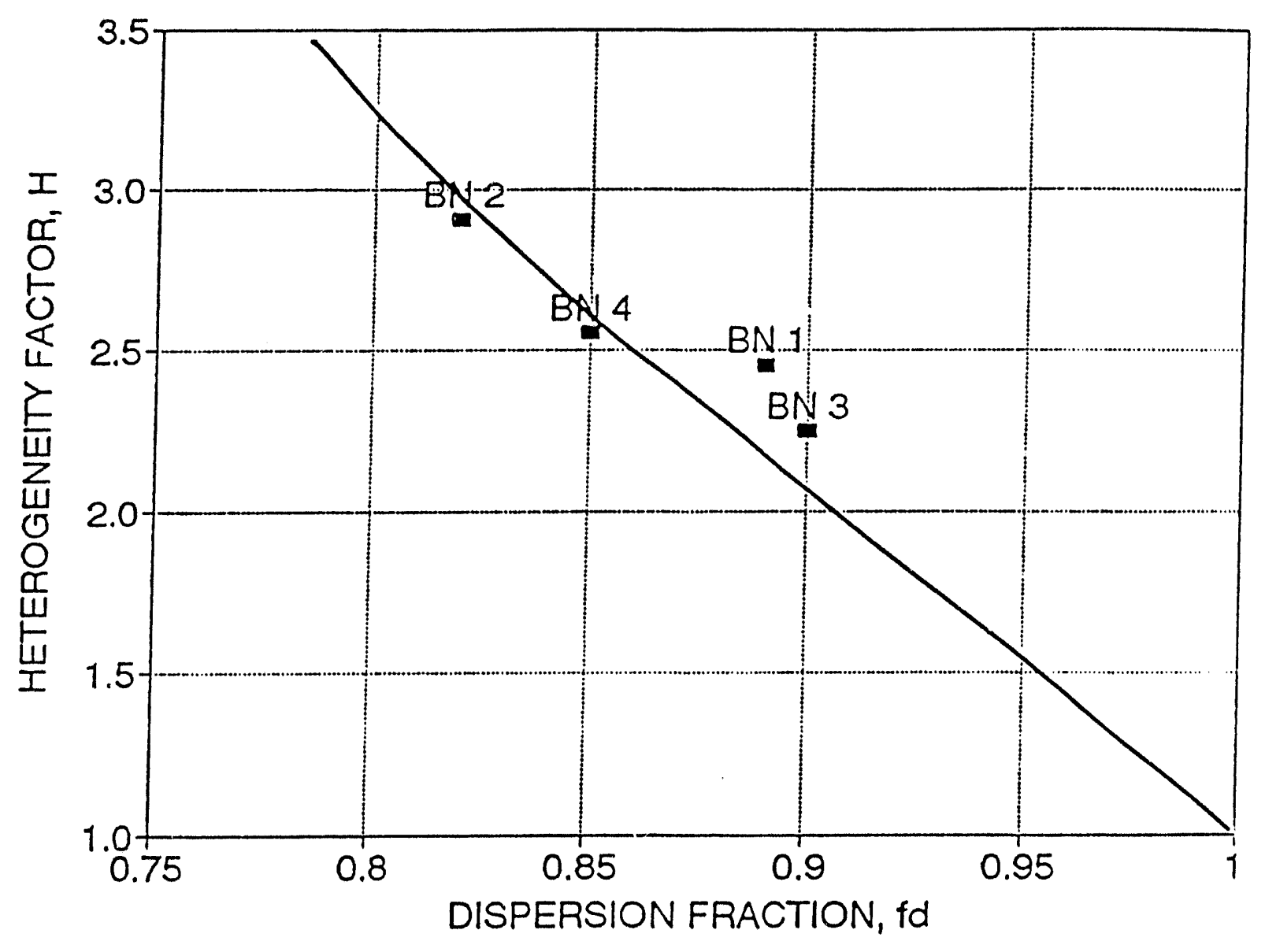

Figure 7.4: Dispersion fraction as a function of heterogeneity factor in 1" diameter Brown samples 


\subsubsection{Defining the Mass Transfer Coefficient (M)}

According to the model of stagnant (or dead end) pore volume employed by Goodknight et al. (1960), Coats and Smith (1964) derived the following expression for the mass transfer coefficient at a steady state process:

$$
M=\frac{\left(1-f_{d}\right) A_{s}}{V_{s} l_{s}} D_{0}
$$

where:

$V_{1}=$ volume of the stagnant space,

$\mathrm{A}_{1}=$ cross-sectional area of the neck leading to the stagnant pore space, and

$1_{1}=$ length of the neck leading to the stagnant space.

Eq. (7.4) indicates that the mass transfer coefficient is proportional to the diffusivity of the fluids used. Thomas et al. (1963) have indicated this fact in their experiments. Thus the high diffusing fluids should be avoided in running dispersion measurements. In addition, almost all literature has indicated that $M$ is velocity-dependent. To obtain the consistent dispersion measurements, all tests should be conducted with the same fluids and the same velocity. Slight variations in velocity were indicated in measuring the concentration profiles on the sandstone core samples in the previous chapter because of the variations in porosity values and fluctuation of the pump rate. Since these variations are small, their effect on dispersivity calculation can be ignored.

Furthermore, Eq.(7.4) also says that the mass transfer coefficient $M$ is also proportional to the diffusion fraction $\left(1-f_{d}\right)$. For the same type of core samples (for example from the same group), the ratio $M /\left(1-f_{d}\right)$ should be a constant. In Figure 7.5, this point is supported by the experimental data. Since the same fluids (methanol and toluene) were used for all tests in this study, the constant slope of the straight line in Figure 7.5 indicates that the samples in the same group have similar stagnant pore spaces. Different slopes of the straight lines represent different parameters for the stagnant pore spaces. Two straight lines can be drawn through all data, one passing through the other four groups of samples. By comparing this figure with the heterogeneity factors listed in Table 6.2, it is found that the samples in group \#1 have relatively low heterogeneities, and the samples in the other four groups have relatively higher heterogeneities. From this fact it probably can be concluded that the parameters for describing the stagnant pore spaces are directly related to the rock heterogeneities. In another word, the higher the heterogeneity of the rock, the larger the stagnant pore volume. 


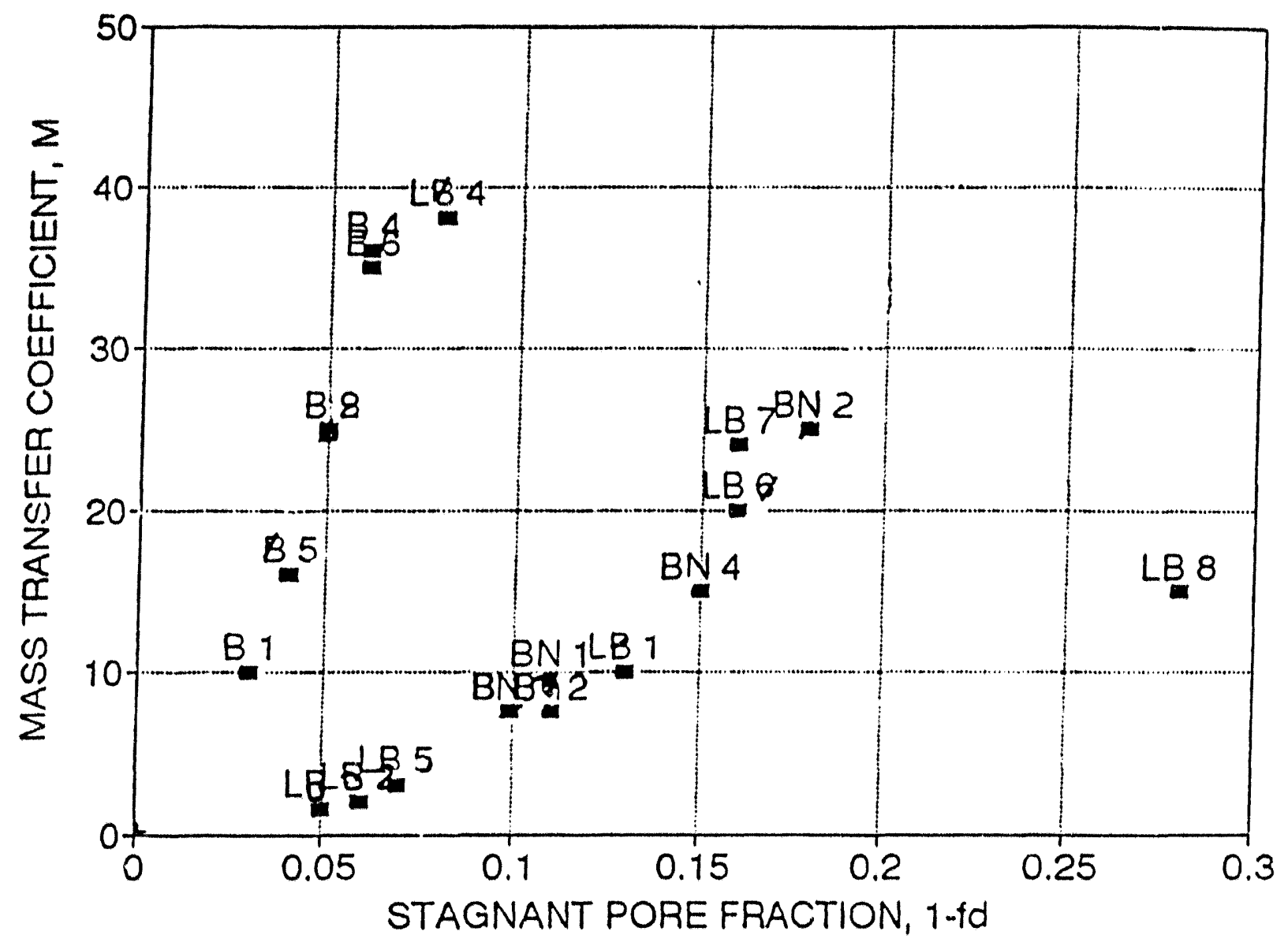

Figure 7.5: Mass transfer coefficient $(M)$ as a function of stagnant pore fraction in Berea and Brown sandstone samples 


\subsection{Determining the "Standard" Dispersivity}

\subsubsection{Matching Concentration Profiles}

After the dispersion fraction and the mass transfer coefficient are determined, the measured concentration profile can be easily well matched with the dispersion capacitance model by adjusting the simple dispersion coefficient. A computer program was developed for this purpose. The fitting parameters for all the concentration profiles obtained experimentally in this study are summarized in Table 7.1.

\subsubsection{Defining the Equivalent Dispersivity}

Three parameters: dispersion coefficient $\left(D_{\mathcal{L}}\right)$, dispersion fraction $\left(f_{d}\right)$, and mass transfer coefficient $(M)$ are obtained for each core sample. However, it is still difficult to characterize the core with these three parameters. It would be much easier to use one equivalent parameter, instead of three parameters, to represent the characteristic of the sample. For this purpose, the results obtained by Yellig and Baker (1981) are used here to convert the three fitting parameters into one equivalent parameter.

Differentiating Eq.(2.16) with respect to time and then substituting the resulting expression for $\partial \mathrm{C}^{\circ} / \partial \mathrm{C}$ into Eq.(2.15), the dispersion capacitance model equation reduces to

$$
D_{L}\left[1+\frac{\left(1-f_{d}\right)^{2}}{\left.\int \frac{D_{L} M}{V_{c}^{2}}\right]} \frac{\left(\frac{\partial^{2} C^{\cdot}}{\partial x^{2}}\right)}{\left(\frac{\partial^{2} C}{\partial x^{2}}\right)}\right] \frac{\partial^{2} C}{\partial x^{2}}-V_{c} \frac{\partial C}{\partial x}=\frac{\partial C}{\partial t}
$$

The above equation is analogous to the convection dispersion model (Eq.2.2), with an equivalent dispersion coefficient, $D_{c}$, which is defined by

$$
D_{C}=D_{L}\left[1+\frac{\left(1-f_{d}\right)^{2}}{\left(\frac{D_{L} M}{V_{c}^{2}}\right)} \frac{\left(\frac{\partial^{2} C^{0}}{\partial x^{2}}\right)}{\left(\frac{\partial^{2} C}{\partial x^{2}}\right)}\right]
$$

The plot of Eq.(7.6), as shown in Figure 7.6, indicates that the equivalent dispersion coefficient increases as both dispersion fraction and mass transfer coefficient decrease.

Through experimental work, Baker (1977) has shown that the equivalent dispersion 
Table 7.1: Fitting Parameters for Matching Concentration Profiles and the Resultant Dispersivities

\begin{tabular}{|c|c|c|c|c|c|}
\hline $\begin{array}{c}\text { Core } \\
\text { Number }\end{array}$ & $\begin{array}{c}Q \\
(\mathrm{Cc} / \mathrm{s})\end{array}$ & $f_{d}$ & $M$ & $\begin{array}{c}D_{L} \\
(\mathrm{Cm} / \mathrm{s})\end{array}$ & $\begin{array}{c}\alpha_{\mathrm{t}} \\
(\mathrm{cm})\end{array}$ \\
\hline$B \# 1$ & 0.0089 & 0.97 & 0.00010 & 0.0015 & 0.242 \\
\hline$B \# 2$ & 0.0093 & 0.95 & 0.00025 & 0.0007 & 0.169 \\
\hline$B \# 3$ & 0.0097 & 0.95 & 0.00025 & 0.0011 & 0.210 \\
\hline$B \# 4$ & 0.0092 & 0.94 & 0.00036 & 0.0012 & 0.221 \\
\hline$B \# 5$ & 0.0093 & 0.96 & 0.00016 & 0.0011 & 0.210 \\
\hline$B \# 6$ & 0.0087 & 0.94 & 0.00035 & 0.0012 & 0.224 \\
\hline$B \# 10$ & 0.0090 & 0.90 & 0.000075 & 0.0015 & 1.442 \\
\hline$B \# 11$ & 0.0100 & 0.90 & 0.000075 & 0.0018 & 1.458 \\
\hline$B \# 12$ & 0.0086 & 0.89 & 0.000085 & 0.0009 & 1.385 \\
\hline$L B \# 1$ & 0.0105 & 0.87 & 0.00010 & 0.0003 & 0.885 \\
\hline$L B \# 2$ & 0.0097 & 0.94 & 0.00005 & 0.0002 & 0.865 \\
\hline$L B \# 3$ & 0.0096 & 0.95 & 0.000015 & 0.0002 & 0.842 \\
\hline$L B \# 4$ & 0.0097 & 0.92 & 0.000038 & 0.0003 & 0.873 \\
\hline$L B 5$ & 0.0097 & 0.93 & 0.00003 & 0.0003 & 0.831 \\
\hline
\end{tabular}




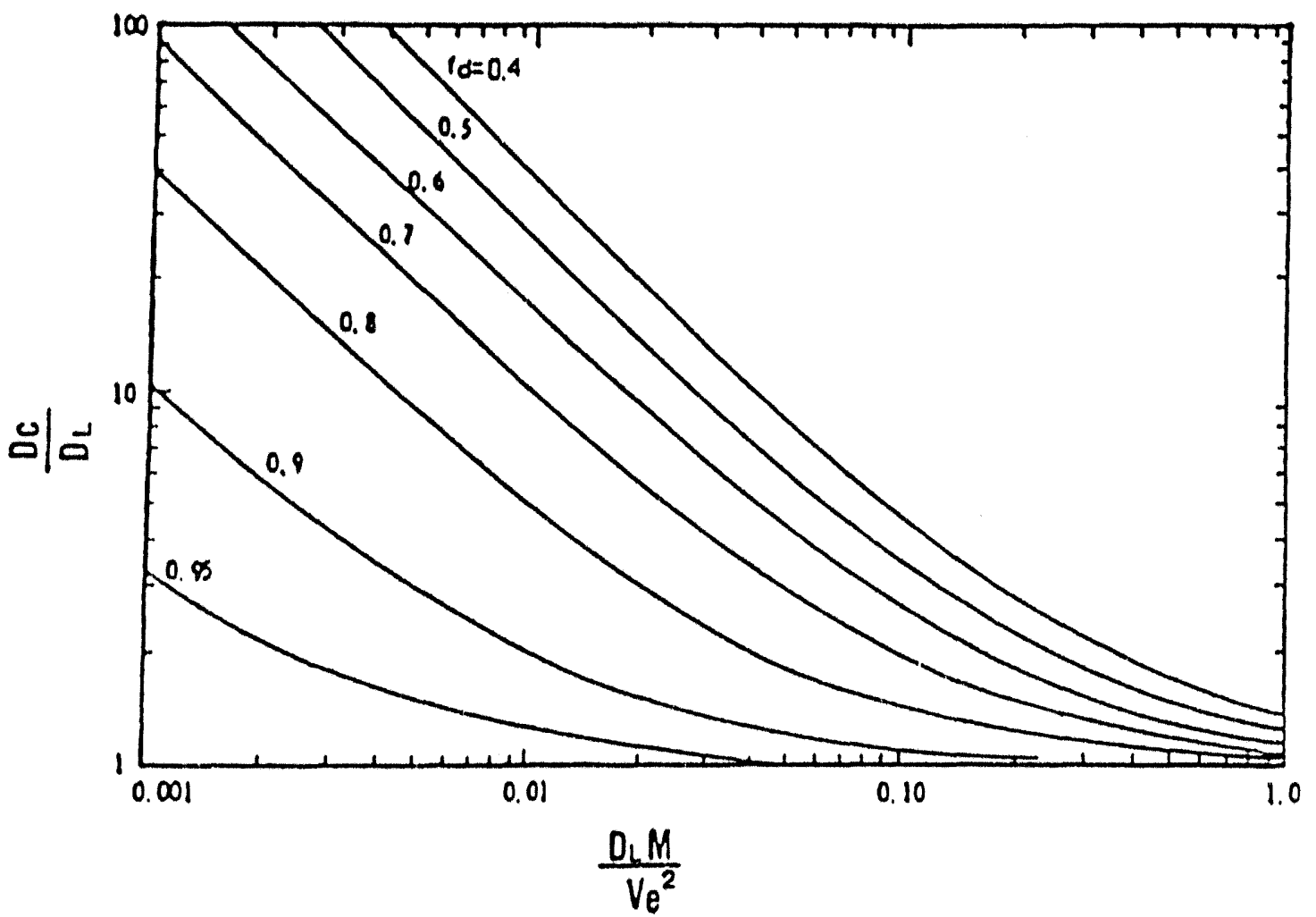

Figure 7.6: Effects of dispersion fraction and mass transfer coefficient on the equivalent dispersion coefficient (Baker, 1977) 
coefficient and mass transfer coefficient were velocity dependent, while the dispersion fraction was relatively independent of velocity.

By assuming that the ratios $\partial^{2} C^{*} / \partial x^{2}$ and $\partial^{2} C / \partial x^{2}$ are equal (at a sufficiently large travel distance, $L$ ), the equivalent dispersivity, $\alpha_{c}$, is then defined as

$$
\alpha_{c}=\alpha_{L}+\frac{\left(1-f_{d}\right)^{2}}{\left[\frac{M}{V_{c}}\right]}
$$

This equation integrates the three fitting parameters into one single parameter. Since the dispersion fraction, $f_{d}$, is velocity independent and the mass transfer coefficient is velocity dependent, the equivalent dispersivity defined by Eq. (7.7) should be independent of fluid flow velocity. With this parameter, one should be able to characterize the dispersion process in porous media.

Using the three fitting parameters obtained from the matching process, the equivalent dispersivity was then calculated using Eq.(7.7) for each core sample. These results are also presented in Table 7.1.

\subsubsection{Effect of the Multiple Solutions}

As pointed out earlier, multiple solutions can be obtained from the dispersion capacitance equation. Based on Eq.(7.7), it seems that the multiple solutions could result in the same equivalent dispersivity value. Figure 7.7 shows an example where the concentration profile for Core B\#4 was satisfactorily matched with two different sets of fitting parameters.

In Figure 7.7a, the fitting parameters used are $D_{L}=0.0012, f_{d}=0.94$, and $M=$ 0.00036 , the resultant equivalent dispersivity is $\alpha_{c}=0.221 \mathrm{~cm}$. In Figure $7.7 \mathrm{~b}$, the fitting parameters used are $D_{L}=0.0016, f_{d}=0.98$, and $M=0.000065$, the resultant equivalent dispersivity is $\alpha_{\mathrm{b}}=0.224 \mathrm{~cm}$. If the third digit is rounded off, the two equivalent dispersivity numbers would be considered the same.

\subsection{Relating the Parameter $\sigma_{\mathrm{B}}$ to the heterogeneity Factor $\mathbf{H}$}

In chapter 3, dispersivity has theoretically been related to the conventional rock properties as indicated in Eq. (3.33). For a quick reference, Eq. (3.33) is presented here for $p=q=1$ : 


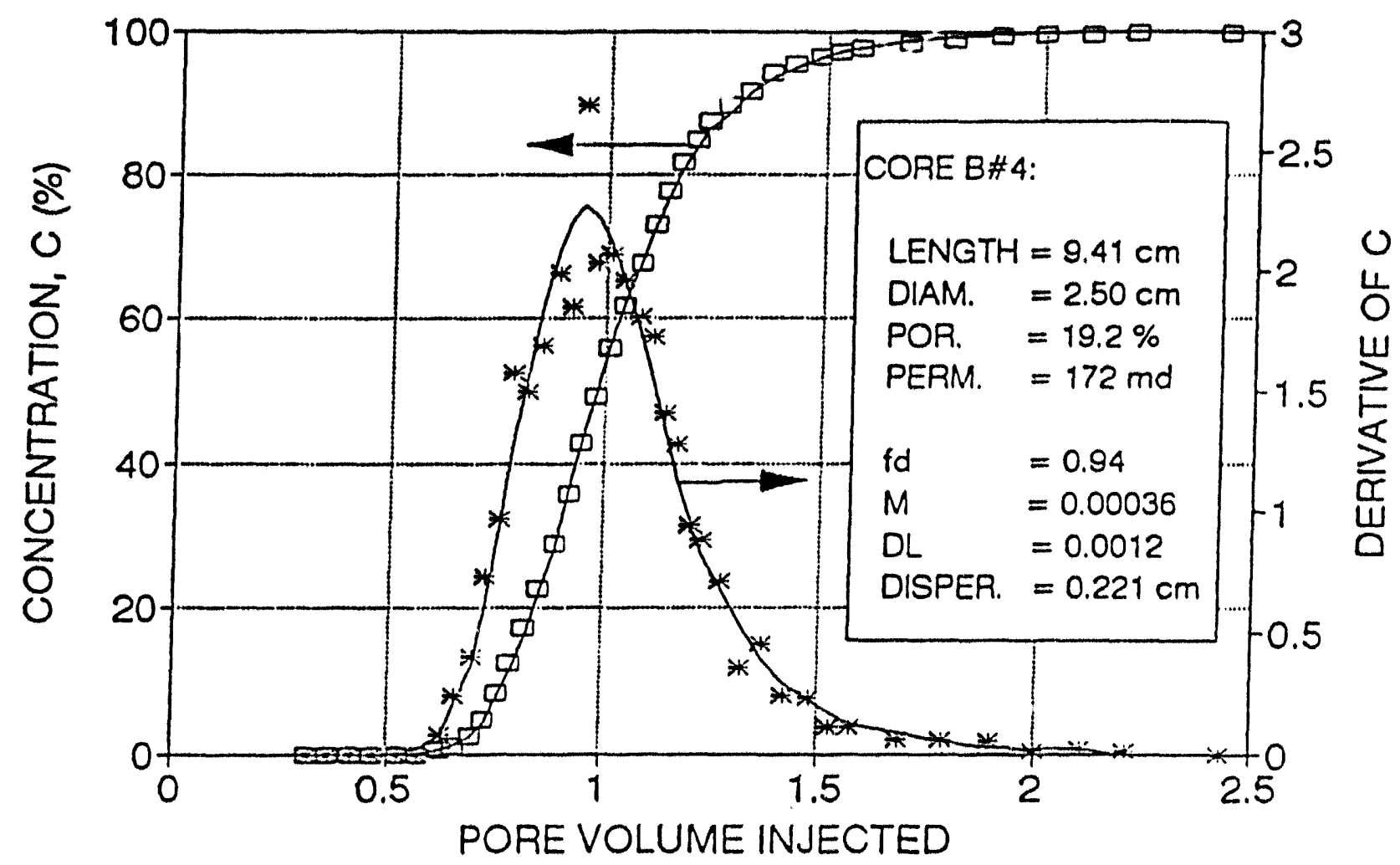

Figure 7.7a: Multiple solutions to the capacitance model resulting in almost the same dispersivity 


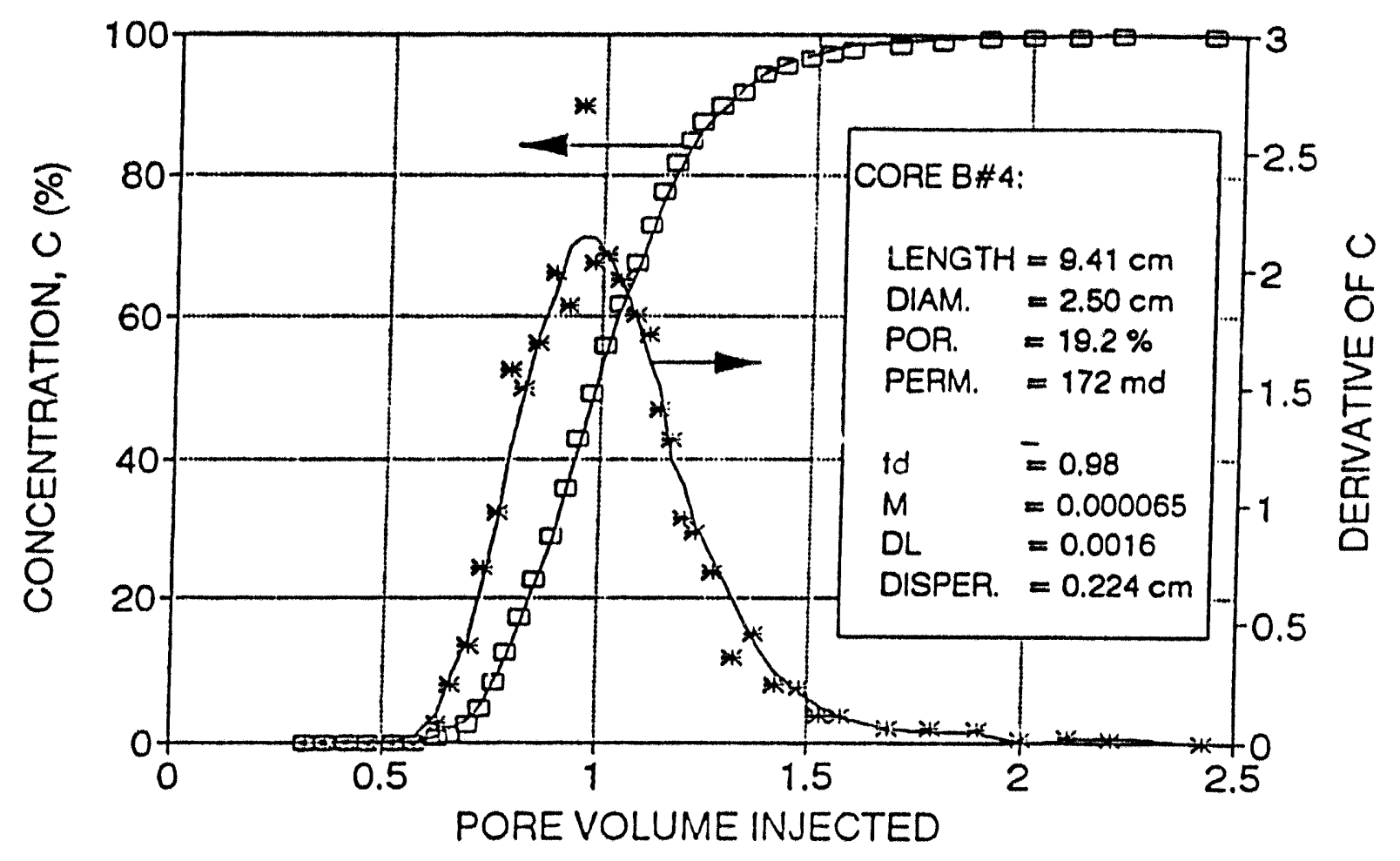

D EXP. - MODEL * EXP. - MODEL

Figure 7.7b: Multiple solutions to the capacitance model resulting in almost the same dispersivity 


$$
\alpha_{D}=6 c \sigma_{H} \tau\left(\frac{1-\phi}{\phi}\right) \sqrt{\frac{R_{0}}{\epsilon} \frac{K}{\phi}}
$$

In some literature, the parameter $\sigma_{\mathrm{H}}$ is also called the heterogeneity factor. To differentiate this parameter with the heterogeneity factor $(H)$ defined in the previous chapter, $\sigma_{\mathrm{H}}$ is called the heterogeneity-related parameter in this chapter.

To use the consistent parameters with the literature, both $\sigma_{\mathrm{H}}$ and $\mathrm{H}$ are also used in this chapter. There is some confusion because both parameters are related to rock heterogeneity. However, due to the different definitions and physical meanings, different parameters should be used. The parameter $\sigma_{\mathrm{H}}$ is a parameter which reflects the effect of rock heterogeneity on dispersion, while the parameter $H$ is defined as the heterogeneity factor which reflects the effect of rock heterogeneity on the miscible displacement efficiency. Since both $\sigma_{H}$ and $\mathrm{H}$ are related to rock heterogeneity, they should be related to each other.

As indicated in Figure 7.8, the experimental data on Berea and Brown sandstones have indicated that dispersivity increases exponentially as the heterogeneity factor $\mathbf{H}$ increases. This figure indicates that the dispersion measured in natural consolidated sandstones is larger than one might have suspected from the heterogeneity caused by the particle size distribution alone. This relationship can be expresses in the form of

$$
\alpha_{D}=c e^{H}
$$

where $\mathrm{c}$ is a constant and $\mathrm{H}$ is the heterogeneity factor defined in the last chapter.

Based on the above equation, the following relationship between the parameter $\sigma_{\mathrm{H}}$ and the heterogeneity factor $(H)$ is proposed here:

$$
\sigma_{H}=c^{\prime} e^{H}
$$

where $c^{\prime}$ is a constant.

By using the "mixing cell" theory, several investigators including Aris and Amundson (1957), Carberry (1958), and Prausnitz (1958) have obtained $\sigma_{H}=3.5$ for random packs. If the sand packs can be considered as homogeneous materials, i.e., $H=1$, then Eq. (7.10) give $c^{\prime}=1.2876$. Substituting this value into Eq. (7.8) yields $c=0.64$, which is close to 0.5 obtained by Aris and Amundson (1957). It is surprising that the constant $\mathrm{c}$ has been found to be about 15 for all Berea and Brown sandstone samples studied in this work. This is not to say that 15 is a universe value for the constant c. A different 


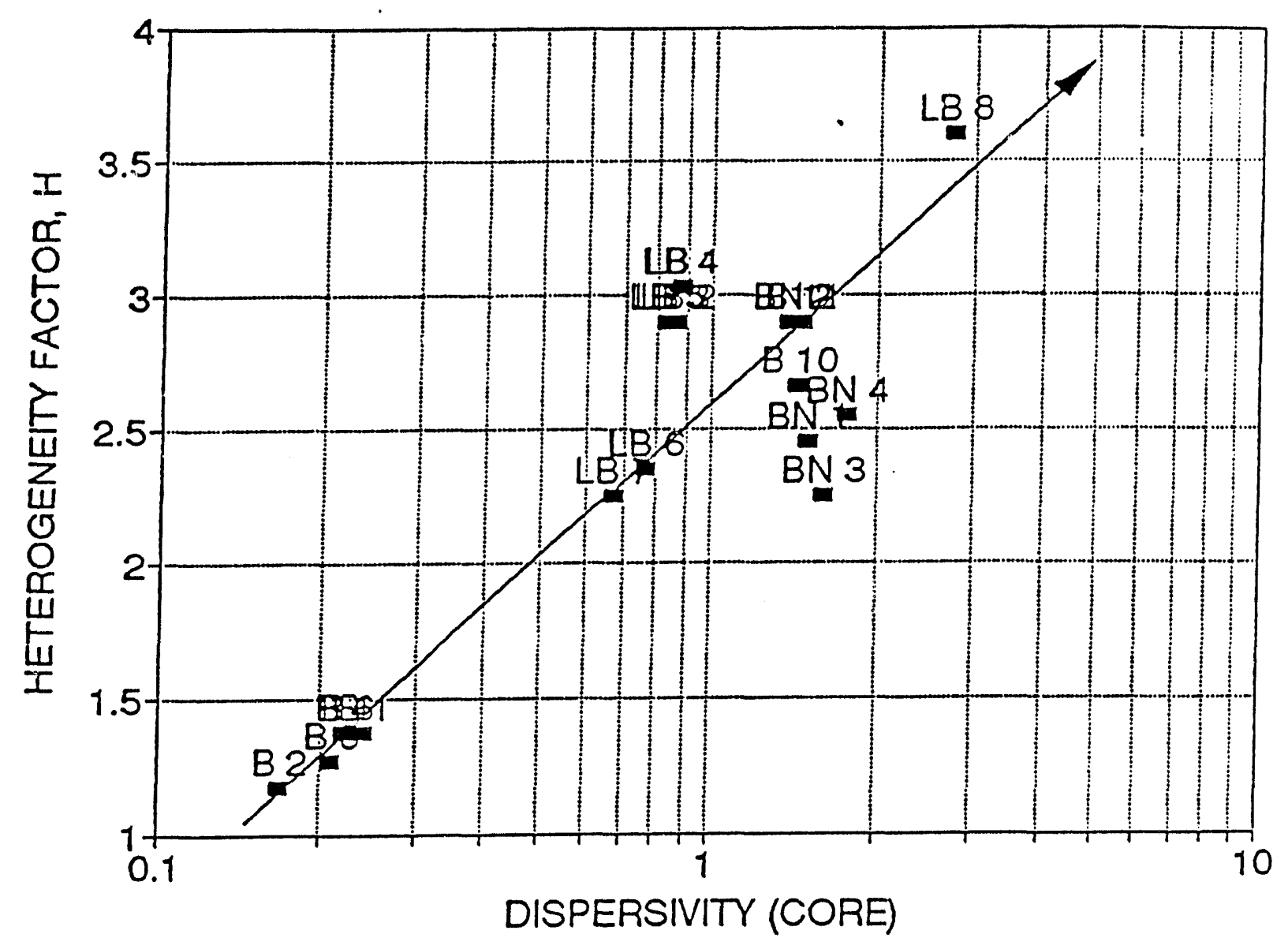

Figure 7.8: Dispersivity versus heterogeneity factor in Berea and Brown sandstone samples 
value could be obtained on other core samples.

Using $c^{\prime}=1.2876$ in Eq.(7.10), the values of $\sigma_{\mathrm{H}}$ for all cores samples are evaluated and the results are presented in Table 7.2.

To compare the $\sigma_{\mathrm{H}}$ values calculated for Berea and Brown sandstone samples in Table 7.2, some $\sigma_{\mathrm{H}}$ values calculated from the literature are listed in Table 7.3. The products of $\sigma_{\mathrm{H}} \mathrm{d}_{\mathrm{p}}$ are obtained from the dispersion measurements on some natural sandstone outcrops. By assuming an average particle size $0.15 \mathrm{~mm}$., the $\sigma_{\mathrm{H}}$ values are calculated from the products of $\sigma_{\mathrm{H}} \mathrm{d}_{\mathrm{p}}$. It can be seen that the $\sigma_{\mathrm{H}}$ values in both Tables 7.2 and 7.3 are very similar for sandstone samples.

\subsection{Comparison of Laboratory Measured Dispersivity with Theoretical Dispersivity}

With the parameter $\sigma_{\mathrm{H}}$, constant $\mathrm{c}$, and rock physical properties including porosity, formation factor (tortuosity) and permeability, the theoretical dispersivity values were calculated using Eq. (7.8). The results are also presented in Table 7.2 as the last column.

Figure 7.9 compares the laboratory data with the theoretical predictions. With a correlation coefficient of 0.98 , the theoretical equation (Eq. (7.8) is applicable to predict the rock dispersivities in single-phase miscible displacement systems. Since those rock parameters can be effectively determined from well logs, Eq.(7.8) is the basic equation for estimating rock dispersivities from well logs.

Through the above correlations and discussions, it can be concluded that the theoretical equation, Eq.(7.8), is valid for predicting the rock dispersivities in singlephase miscible displacement cases. With the tortuosity, heterogeneity factor, porosity, and permeability determined from well logs, the rock dispersivity can be predicted by Eq.(7.8).

Meanwhile, the equivalent dispersivity determined from the Coats-Smith capacitance model is also considered to be appropriate to represent the measured S-shaped concentration profile. With the equivalent dispersivity parameter, the dispersion phenomena in a porous medium can be characterized.

Further, since dispersivity is related to so many rock parameters as indicated by Eq. (7.8), no simple equivalence between dispersivity and any one rock parameter should be expected. Instead, dispersivity should be considered to be equally important as compared to the conventional petrophysical properties such as porosity, tertuosity, and permeability. 
Tabie 7.2: Rock Heterogeneity Factors and Theoretical Dispersivities

\begin{tabular}{|c|c|c|c|}
\hline Core No. & $\mathrm{H}$ & $\sigma_{H}$ & $\alpha_{\text {th }}$ \\
\hline B\#1 & 1.378 & 5.11 & 0.23 \\
\hline$B \# 2$ & 1.174 & 4.17 & 0.17 \\
\hline$B \# 3$ & 1.272 & 4.59 & 0.20 \\
\hline $\mathrm{B} \# 4$ & 1.378 & 5.11 & 0.22 \\
\hline B\# 5 & 1.272 & 4.59 & 0.21 \\
\hline$B \neq 6$ & 1.378 & 5.11 & 0.22 \\
\hline$B \# 10$ & 2.662 & 18.44 & 1.28 \\
\hline$B \# 11$ & 2.899 & 23.38 & 1.67 \\
\hline$B \# 12$ & 2.899 & 23.38 & 1.32 \\
\hline LB\#1 & 3.03 & 26.65 & 0.87 \\
\hline LB\#\# 2 & 2.90 & 23.40 & 0.81 \\
\hline LB\# 3 & 2.90 & 23.40 & 0.81 \\
\hline LB\# 4 & 3.03 & 26.65 & 0.88 \\
\hline LB\# 5 & 2.90 & 23.40 & 0.86 \\
\hline
\end{tabular}


Table 7.2: (Continued)

\begin{tabular}{|c|c|c|c|}
\hline Core NO. & $\mathrm{H}$ & $\sigma_{\mathrm{H}}$ & $\alpha_{\mathrm{W}}$ \\
\hline \hline LB\#6 & 2.25 & 13.50 & 0.78 \\
\hline LB\#7 & 2.16 & 12.22 & 0.70 \\
\hline LB\#8 & 3.45 & 47.12 & 2.81 \\
\hline BN\#1 & 2.45 & 14.92 & 1.59 \\
\hline BN\#2 & 2.90 & 23.40 & 1.23 \\
\hline BN\#3 & 2.25 & 12.22 & 1.49 \\
\hline BN\#4 & 2.55 & 16.49 & 1.65 \\
\hline
\end{tabular}

Table 7.3: $\sigma_{\mathrm{H}}$ Values for Natural Sandstones

\begin{tabular}{||c|c|c|c|}
\hline \multicolumn{1}{|c|}{ Source } & Rock & $\begin{array}{c}\sigma_{\mathrm{H}} \cdot \alpha_{\mathrm{p}} \\
(\mathrm{cm})\end{array}$ & $\begin{array}{c}\sigma_{\mathrm{H}} \\
\left(\alpha_{\mathrm{P}}=0.15 \mathrm{~mm}\right)\end{array}$ \\
\hline \hline Grane \&Gardner (1961) & Berea & 0.25 & 16.7 \\
\hline Brigham et al. (1961) & Berea & 0.39 & 26.0 \\
& Torpedo & 0.17 & 11.3 \\
\hline Ramondi et al. (1959) & Berea & 0.46 & 30.7 \\
\hline Handy (1959) & Bnise & 0.55 & 36.7 \\
\hline
\end{tabular}




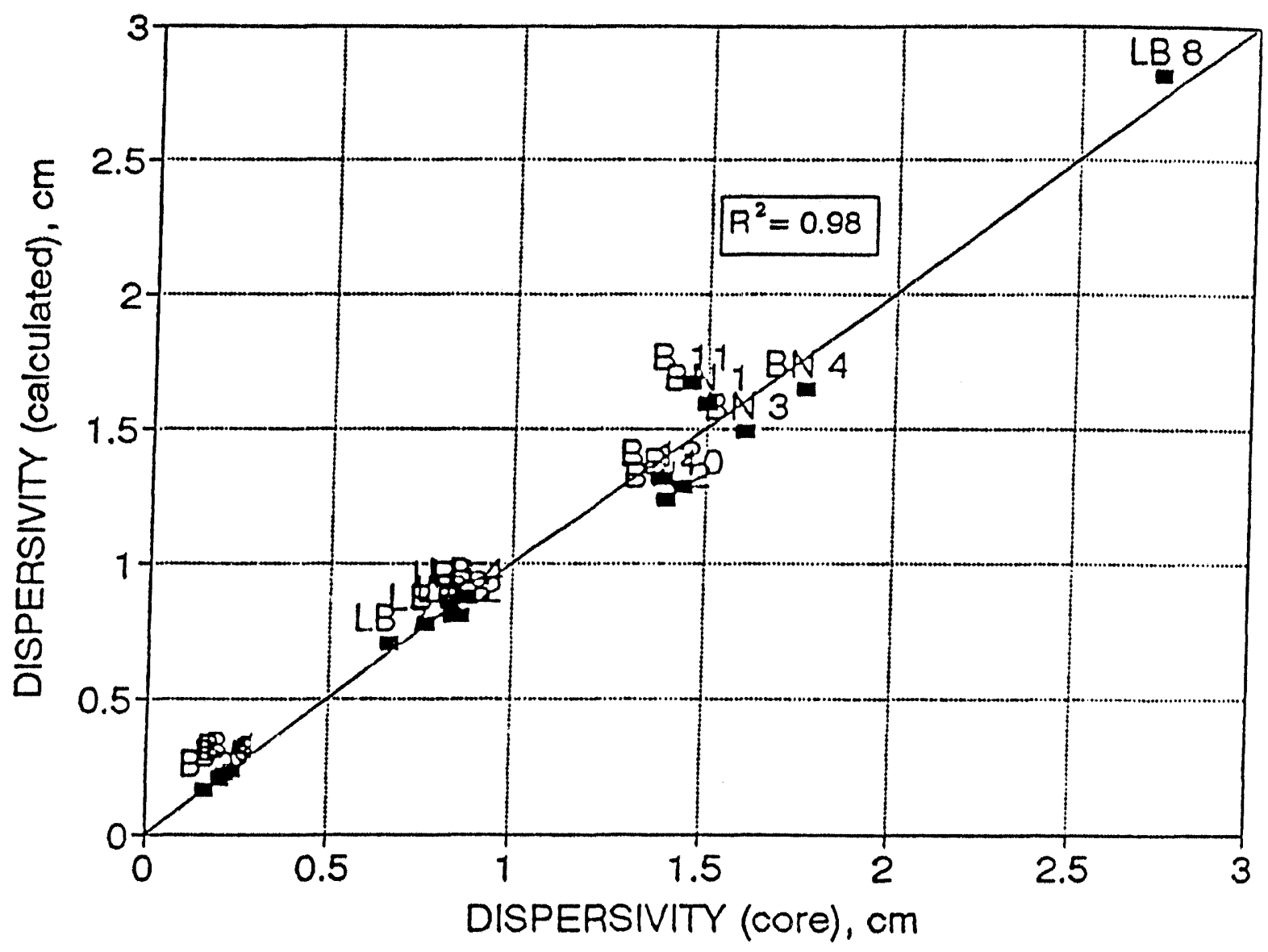

Figure 7.9: Comparison of laboratory measured dispersivity with theoretical prediction 


\section{CHAPTER 8}

\section{CONCLUSIONS}

Through theoretical analyses and experimental studies presented in the preceding chapters, the following conclusions havis been obtained:

(1) The tortuosity factor plays an essential role in connecting dispersivity with conventional rock properties.

(2) Coats-Smith's dispersion-capacitance equation can be used effectively to model the dispersion phenomena in porous media.

(3) The dispersion fraction (or flow fraction) parameter in the dispersion-capacitance equations can be determined from the concentration derivative curve. As a result, a unique solution to the dispersion-capacitance equations can be obtained.

(4) The equivalent dispersivity determined from the three fitting parameters in the dispersion-capacitance equations can be used as a characteristic of a reservoir rock.

(5) A new method was proposed to evaluate rock heterogeneities from the concentration profiles which can be measured through miscible displacement tests in the laboratory.

(6) An approach to estimating rock dispersivity from well logs was presented and was verified by the laboratory experiments. 


\section{NOMENCLATURE}

$A=$ cross-sectional area of the core sample $\left(\mathrm{cm}^{2}\right)$

$A_{1}=$ cross-sectional area available to fluid flow defined by Cornell and Katz $\left(\mathrm{cm}^{2)}\right.$

$A_{1}=$ cross-sectional area of the neck leading to the stagnant pore space $\left(\mathrm{cm}^{2}\right)$

a $=$ a correlation constant

b $=$ a correlation constant

BV = bulk volume of the sample $\left(\mathrm{cm}^{3}\right)$

c = a general constant which has different value in different equations

$c^{\prime}=a$ constant in Eq. $(7.10)$

C = in-situ concentration of flowing fluid

$C_{f}=$ effluent concentration of displacing fluid in flux

$C_{1}=$ surface conductance caused by electric double-layer (ohm ${ }^{-1}$ )

$\mathrm{C}_{\text {ast }}=$ salt concentration in the saturation fluid

$C_{t}=$ overall conductance of a porous medium (ohm ${ }^{-1}$ )

$C^{*}=$ in-situ concentration of stagnant fluid

$\mathrm{d}=$ diameter of the core sample $(\mathrm{cm})$

$d_{50}=50$ percent particle size $(\mathrm{cm})$

$d_{0}=$ outer diameter of a logging tool $(\mathrm{cm})$

$\mathrm{d}_{\mathrm{p}}=$ particle diameter $(\mathrm{cm})$

$d_{d}=$ separation distance between the flood fronts of concentration $C$ and $C^{*}$

$D=$ general dispersion coefficient $\left(\mathrm{cm}^{2} / \mathrm{sec}\right)$

$D_{c}=$ equivalent dispersion coefficient $\left(\mathrm{cm}^{2} / \mathrm{sec}\right)$

$D_{L}=$ longitudinal dispersion coefficient $\left(\mathrm{cm}^{2} / \mathrm{sec}\right)$

$D_{L^{\prime}}=$ longitudinal dispersion coefficient in a tortuous capillary $\left(\mathrm{cm}^{2} / \mathrm{sec}\right)$

$D_{\mathrm{m}}=$ effective molecular diffusivity of fluids in a porous medium, $\left(\mathrm{cm}^{2} / \mathrm{sec}\right)$

$D_{0}=$ molecular diffusivity of fluid $\left(\mathrm{cm}^{2} / \mathrm{sec}\right)$

erf $=$ error function

erfc $=$ complementary error function

f $=$ flow fraction

$f_{d}=$ dispersion (or mixing) fraction

$f_{1}=$ solvent fraction in effluent flux

$F=$ electrical formation factor

$h_{d}=$ average thickness of the measuring electrir current layer of the deep-investigating logging tool $(\mathrm{cm})$

$\mathrm{H}$ = rock heterogeneity factor

I $=$ pore volume injection $\left(=V_{i} / V_{p}\right)$

$\mathbf{J}=\mathbf{I} / \mathbf{f}$ 
$K_{0}=$ Kozeny shape factor ranging from 2 to 3

$\mathbf{K}=$ absolute permeability (md)

$\mathbf{K}_{d}=$ calibration coefficient of the deep-investigating logging tool

$\mathbf{K}_{\mathbf{1}}=$ calibration coefficient of the shallow-investigating logging tool

$\mathbf{K}_{s 0}=$ permeability value at the 50 percent probability (md)

$K_{\mu .1}=$ permeability at the 84.1 percent probability (md)

$\mathrm{u}_{1}$ = length of the neck leading to the stagnant volume $(\mathrm{cm})$

$\mathrm{L}=$ length of the core sample $(\mathrm{cm})$

$L_{0}=$ critical system length $(\mathrm{cm})$

$\mathbf{L}_{\mathbf{w}}=$ the distance from wellbore to the equivalent zero potential point for a deepinvestigating logging tool $(\mathrm{cm})$

$L_{c}=$ length of a tortuous path in a porous medium $(\mathrm{cm})$

$L_{t}=$ the distance from wellbore to the equivalent zero potential point for a shallowinvestigating logging tool $(\mathrm{cm})$

$M=$ mass transfer coefficient $\left(\mathrm{sec}^{-1}\right)$

$\mathrm{n}_{\mathrm{K}}=$ an exponent constant

$\mathrm{n}_{\mathrm{V}}=$ velocity exponent

$p=a$ correlation constant

$P=$ pressure (atm)

$P_{0}=$ peclet number

$\mathrm{PV}=$ pore volume of a porous medium $\left(\mathrm{cm}^{3}\right)$

$q=a$ correlation constant

$\mathrm{Q}=$ flow rate $\left(\mathrm{cm}^{3} / \mathrm{sec}\right)$

$\mathbf{r}_{0} \quad=$ measured resistance of a sample (ohm)

$\mathrm{r}_{\mathrm{b}}=$ hydraulic radius $(\mathrm{cm})$

$R_{0}=$ measured resistivity of a core sample (ohm-m)

$R_{d}=$ resistivity reading from the deep-investigating logging tool (ohm-m)

$R_{d s 0}=$ particle size Reynolds number

$R_{k}=$ permeability Reynolds number

$\mathbf{R}_{\mathrm{mf}}=$ mud filtrate resistivity (ohm-m)

$R_{0}=$ resistivity of the rock that is fully saturated rock with brine (ohm-m)

$R_{\mathrm{Tw}}=$ relative resistivity to the wetting phase

$R_{1}=$ resistivity reading from the shallow-investigating logging tool (ohm-m)

$R_{\mathrm{sF}}=$ micro-log resistivity (ohm-m)

$R_{1}=$ resistivity of a partially-saturated medium (ohm-m)

$\mathbf{R}_{\mathrm{w}}=$ resistivity of the brine (ohm-m)

$S=$ particle surface area per unit volume $\left(\mathrm{cm}^{2}\right)$

$S_{0}=$ specific surface area of the particles

$S_{1}=$ solvent saturation

$S_{\mathrm{W} \mid}=$ connate water saturation of a rock

$\mathrm{t}=$ time (sec)

$T=$ tortuosity factor

$T_{b}=$ breakthrough time of injected fluid at the outlet (sec) 
$u_{c}=$ average velocity in a capillary $(\mathrm{cm} / \mathrm{sec})$

$V$ Darcy velocity $(\mathrm{cm} / \mathrm{sec})$

$V^{\prime}=$ pore velocity modified by Carman $(\mathrm{cm} / \mathrm{sec})$

$V_{D p}=$ Dykstra-Parsons permeability variation coefficient

$V_{0}=$ interstitial velocity in a porous medium $(\mathrm{cm} / \mathrm{sec})$

$V_{1}=$ Volume of displacing fluid injected $\left(\mathrm{cm}^{3}\right)$

$V_{L z}=$ Lorenz permeability variation coefficient

$V_{p}=$ pore volume of the porous medium $\left(\mathrm{cm}^{3}\right)$

$V_{1}=$ volume of the stagnant pores $\left(\mathrm{cm}^{3}\right)$

$W_{d}=$ weight of a dry core sample (gr)

$W_{w}=$ weight of a fully wet core sample (gr)

GREEK LETTERS

$\alpha_{c}=$ equivalent dispersivity (cm)

$\alpha_{D}=$ dispersivity $(\mathrm{cm})$

$\alpha_{D, d}=$ dynamic dispersivity (cm)

$\alpha_{D_{\text {S }}}=$ geometric dispersivity (cm)

$\alpha_{L}=$ "standard" dispersivity of a rock (cm)

$\varepsilon \quad=$ particle surface factor

$\lambda=$ volume modifying function parameter

$\lambda_{j}=\lambda$ values at the $j \%$ concentration of displacing fluid $(j=10,20,80$, and 90$)$

$\mu \quad=$ kinematic fluid viscosity (cp)

$\rho=$ porosity of the rock (\%)

$\rho=$ density of a fluid $\left(\mathrm{gr} / \mathrm{cm}^{3}\right)$

$Q_{w}=$ density of brine $\left(\mathrm{gr} / \mathrm{cm}^{3}\right)$

$\sigma_{\mathrm{H}}=$ heterogeneity-related parameter

$\tau=$ tortuosity

$\psi=$ dimensionless coefficient in Eq.(2.30)

$x=$ dimensionless coefficient in Bq.(2.3) 
CONSTANTS IN COATS AND BMITH MODEL SOLUTIONS

$$
\begin{aligned}
& a_{1}=1+\sqrt{\rho} \cos (\theta / 2)-\beta \sqrt{\rho} \sin (\theta / 2) \\
& a_{2}=\beta(1+\sqrt{\rho} \cos (\theta / 2))+\sqrt{\rho} \sin (\theta / 2) \\
& a=\frac{L M}{V_{0}} \\
& b=\frac{a f}{(1-f)} \\
& u=1+\frac{4}{\gamma}\left(1+\frac{b a+a\left(1+\beta^{2}\right)}{(1+b)^{2}+\beta^{2}}\right) \\
& v=\frac{4 \beta}{\gamma}\left(1+\frac{a b}{(1+b)^{2}+\beta^{2}}\right) \\
& \rho=\sqrt{u^{2}+v^{2}} \\
& w=\frac{\gamma}{2} \sqrt{\rho} \sin (\theta / 2) \\
& \theta=\tan { }^{-1}(v / u) \\
& \Gamma_{1}=\frac{a_{1}}{2}\left(1+\frac{\gamma v}{4 w}\right)+\frac{a_{2} w}{\gamma} \\
& \Gamma_{2}=\frac{a_{2}}{2}\left(1+\frac{\gamma v}{4 w}\right)-\frac{a_{1} w}{\gamma} \\
& \hline
\end{aligned}
$$




\section{REFERENCES}

Alpay, O.A. (1972): "A Practical Approach to Defining Reservoir Heterogeneity", Loumal of Petroleum Technology, July, p. 841-848.

Amyx, J.W., Bass, D.M., Jr., and Whitting, R.L. (1960): "Petroleum_Reservoir Engineering (Physical Properties)", McGraw Hill, New York.

Anderson, M.P. (1979): "Using Models to Simulate the Movement of Contaminants Through Groundwater Flow Systems", Critical Reviews in Environmental Controls, Vol.9, No.2, p. 97-156.

Archie, G.E. (1942): "The Electrical Resistivity Log as an Ald in Determining Some Reservoir Characteristics", Transaction of American Institute of Mechanical Engineers, Vol.146, p. 54-62.

Aris, R. (1956): "On the Dispersion of a Solute in a Fluid Flowing Through a Tube", Proc. Reyal Soci of London, Series A. Vol.235, p. 67-77.

Aris, R. and Amundson, N.R. (1957): "Some Remarks on Longitudinal on Miscible Flooding", ALCHE Joumal, Vol.3, p. 280-282.

Aris, R. (1959): "The longitudinal Diffusion Coefficient in Flow Through a Tube with Stagnant Pockets", Chemical Engineering Science, Vol. 11, p. 194-198.

Baker, L.E. (1977): "Effects of Dispersion and Dead-End Pore Volume in Miscible Flooding",Society of Petroleum Engineers Joumal, p. 219-227.

Batycky, J.P., Maini, B.B., and Fisher, D.B. (1982): "Simulation of Miscible Displacement in Full-Diameter Carbonate Cores", Society of Petroleum Engineers Joumal, p. 647-657.

Bear, J. (1960): "The Transition Zone between Fresh and Salt Waters in Coastal Aquifers, Ph.D. Dissertation, University of California, Berkeley, CA..

Bear, J. (1961): "On the tensor Form of Dispersion in Porous Media", Joumal of Geophysical Research, Vol. 66, No. 4, p. 1185-1197. 
Bear, J. (1969): "Hydrodynamic Dispersion", In Elow Through Porous Media, Edited by De Wiest, R.J.M., Academic Press, New York, P. 109-200.

Bear, J. and Bachmat, Y. (1967): "A Generalized Theory on Hydrodynamic Dispersion in Porous Media", I.A.S.H. Symp. Artificial Recharge and Management of Aquifers, Haifa, Israel, IASH, P.N. 72, p. 7-16.

Bikerman, J.J. (1940): "Electrokinetic Equations and Surface Conductance, a Survey of the Diffuse Double Layer Theory of Colloidal Solutions", Earad. Soc. Trans., Vol. 36, p. 154-160.

Blackwell, R.J. (1962): "Laboratory Studies of Microscopic Dispersion Phenomena", Society of Petroleum Engineers Joumal, p. 1-8.

Brigham, W.E., Reed, P.W., and Dew, J.N. (1961): "Experiments on Mixing During Miscible Displacement in Porous Media", Society of Petroleum Engineers Joumal, p. 18.

Brigham, W.E. (1972): "Mixing Equations in Short Laboratory Cores", Society of Petroleum Engineers Joumal, p. 91-99.

Buckley, S.E. and Leverett, M.C. (1942): "Mechanisms of Fluid Displacement in Sands", Trans. ANME, Vol. 146, p. 107-116.

Carberry, J.J. and Bretton, R.H. (1958): "Axial Dispersion of Mass in Flow Through Fixed Beds", American Institute of Chemical Engineering_Joumal, Vol. 4 No. 3, p. 367 375.

Carman, P.C. (1937): "Fluid Flow Through Granular Beds", Trans, of Institute of Chemical Engineering, London, Vol. 15, p. 150-166.

Carman, P.C. (1956): Elow of Gases Through Porous Media, Academic Press Inc., New York.

Chen, S. and Tan, T. (1983): "A New Method of Mud Fluid Invasion Corrections for Dual Laterologs", Ioumal of Well Logging Tech. (in Chinese), Yol. 4, p. 1-12.

Chen, S. and Tan, T. (1985): "Investigations on Calibration Coefficients and Investigation Depth of Laterolog Tools", Presented at the Annual National Electric Logging Conference, Hangzhou, China.

Chen, S.M., Allard, D.R., and Anli, J. (1984): "Factors Affecting Solvent Slug Size Requirements in Hydrocarbon Miscible Flooding", SPE/DOE paper 12636, Presented at the SPE/DOE Fourth Symposium on Enhanced Oil Recovery, Tulsa, Oklahoma. 
Coats, K.H. and Smith, B.D. (1964): "Dead End Pore Volume and Dispersion in Porous Media", Society of Petmoleum Engineers Joumal, p. 73-84.

Collins, R.B. (1976): Elow of Eluids Through Porous Materials, The Petroleum Publishing Company, Tulsa, OK.

Comell, D. and Katz, D.L. (1953): "Flow of Gases Through Consolidated Porous Media", Ind. Enge Chem., Vol. 45, No. 10, P. 2145-2152.

Davis, A.D. (1986): "Deterministic Modeling of Dispersion in Heterogeneous Permeable Modia", Ground Water, Vol. 24, No. 5, p. 609-615.

Deans, H.A. (1963): "A Mathematical Model for Dispersion in the Direction of Flow in Porous Media", Society of Petroleum Engineers Joumal, P. 49-53.

Dewan, J.T. (1983): Essentials of Modem Open-Hole Loe Interpretation, PennWell Books, Tulsa, Oklahoma.

De Witte, L. (1950): "Relations between Resistivities and Fluid Contents of Porous Rock", Oil and Gas Joumal, Vol. 49, P. 120-132.

Domenico, P.A. and Robbins, G.A. (1984): "A Dispersion Scale Effect in Model Calibrations and field Tracer Experiments", Joumal of Hydrology, Vol. 70, P. 123-132.

Dullien, F.A.L., Lai, F.S.Y., and MacDonald, I.F. (1986): "Hydraulic Continuity of Residual Wetting Phase in Porous Media", Joumal of Colloid and Interface Science, Vol. 109 , No. 1, p. 201-218.

Dykstra, H. and Parsons, R.L. (1950): "The Prediction of Oil Recovery by Water flood", Secondary Recovery of Oill in United States, 2nd Edition, API, Now York, p. 160-174.

Evers, J.F. and Iyer, B.G. (1975): "Quantification of Surface Conductivity in Clean Sandstone", Presented at the 5th Formation Bvaluation Symposium of the Canad. Well Logging Society in Calgary, Canada, Paper L.

Freeze, R.A. and Cherry, J.A. (1979): Groundwater, Prentice-Hall, Englewood Cliffs, N.J..

Goodknight, R.c., Klikoff, W.A., and Fatt, I. (1960): "Non-Steady State Fluid Flow and Diffusion in Porous Media Containing Dead End Pore Volume", Jour. Phys. Chem., Vol. 64, p. 1162. 
Greenkom, R.A. and Kessler, D.P. (1970); "Dispersion in Heterogeneous Nonuniform Anisotropic Porous Media", Elow Through Porous Media, Bdited by Nunge, R.J. and Gill, W.N., Amer. Chem. Soc. Publications, Washington D.C., p. 160-177.

Harleman, D.R.F., Mehlhorn, P.F., and Rumer, R.R., Jr. (1963): "dispersionPermeability Correlation in Porous Media", Joumal of the Hydraulic Div. Proc. Amer. Soc. Civil Eng, p. 67-85.

Jensen, J.L. (1986): A Statistical Study of Reservoir Permeability Distributions, Ph.D. Dissertation, University of Texas, Austin, Texas.

Kasraie, M. (1979): "Influence of Rate and Various Immobile Fluid Saturations on Convective Mixing in a Porous Medium", M.S. thesis, The Pennsylvania State University.

Kelkar, B.G. and Gupta, S.P. (1988): "The effect of Small Scale Heterogeneities on the Effective Dispersivity of Porous Media", SPE/DOE paper 17339, Presented at the Sixth SPE/DOE Symposium on Enhanced Oil Recovery, Tulsa, Oklahoma.

Klotz, D. and Moser, H. (1974): "Hydrodynamic Dispersion as Aquifer Characteristic: Model Experiments with Radioactive Tracers", Broc. Series. Isotope Tech. in Groundwater Hydrology. Intern. Atomic Energy Agency aABA), Vol. 12, Vienna, p. 341-355.

Koval, B.J. (1963): "A Method for Predicting the Perfornance of Unstable Miscible displacement in Heterogeneous Media", Society of Petroleum Engineers Joumal, p. 145 154.

Kreft, A. and Zuber, A. (1978): "On the Physical Meaning of the Dispersion Equation and Its Solutions for Different Initial and Boundary Conditions", Chem. Eng. Scil, Vol. 33, p. 1471-1480.

Lake, L.W., Scott, A.J., and Kocurek, G.A. (1986): "Reservoir Characterization for Numerical Simulation", Final Report (DOE/BC/10744-8) to U.S. Department of Energy under contract No. AS19-82BC10744, Bartlesville, Oklahoma.

Lambert, M.E. (1981): "A Statistical Study of Reservoir Heterogeneity", M.S. Thesis, University of Texas, Austin, Texas.

Law, J. (1944): "Statistical Approach to the Interstitial Heterogeneity of Sand Reservoirs", Trans. ADME, Vol. 155, p. 202-222. 
Maini, B.B., Ionescu, E., and Batycky, J.P. (1986): "Miscible Displacement of Residual Oil - Effect of Wettability on Dispersion in Porous Media", Joumal of Canadian Petroleum Technology, Vol. 25, No. 3, p. 36-41.

Menzie, D.E., Dutta, S., and Shadizadeh, R.S. (1988) "A New Method of Coating Oilfield Core for Laboratory Studies", Joumal of Petroleum Technology, p. 643-644.

Menzie, D.E. and Dutta, S. (1989): "Dispersivity as an Oil Reservoir Rock Characteristic", Final report to U.S. Department of Energy, No. DOE/BC/10851-15, Bartlesville, Oklahoma.

Moltz, F.J., Guven, O., and Melville, J.G. (1983): "An Examination of Scale-Dependent Dispersion Coefficients", Ground Water, vol. 21, No. 6, p. 715-725.

Niemann, E.H. (1969): "Dispersion During Flow in Non-Uniform, Heterogeneous Porous Media", M.S. Thesis, Purdue University, Lafayette,Ind.

Ogata, A. and Banks, R.B. (1961): "A Solution of The Differential Equation of Longitutional Dispersion in Porous Media", Geological Survey Professional Paper No. 411-A, United States Department of the Interior, Washington, D.C., pp. 7.

Ogata, A. (1970): "theory of Dispersion in a Granular Medium", U.S. Geological Survey Professional Paper, No. 411-I.

Orlob, G.T. and Radhakrishna, G.N. (1958): "the Effects of Entrapped Gases on the Hydraulic Characteristics of Porous Media", Trans. of American Geophysical Union, Vol. 39, No. 4, p. 640-659.

Patel, K.M. and Greaves, M. (1987): "Surfactant Dispersion in Porous Media", Chem. Eng. Res. Des., Vol. 65, p. 12-22.

Paterson, M.S. (1983): "the Equivalent Channel Model for Permeability and Resistivity in Fluid-Saturated Rocks--- A Reappraisal", Mech. Mater., Vol.2, No. 4, p. 345-352.

Patnode, H.W. and Wyllie, M.R.J. (1950): "The presence of Conductive Solids in Reservoir rocks as a Factor in electric Log Interpretation", Trans. AIME, Vol. 189, p. 47-52.

Perkins, T.K. and Johnston, O.C. (1963): "A Review of Diffusion and Dispersion in Porous Media", Socity of Petrole: in Engineers Joumal, p. 70-84. 
Pfannkuch, H.O. (1972): "On the Correlation of Electrical Conductivity Properties of Porous Systems with Viscous Flow Transport Coefficients", in Eundamentals of Transport Phenomena in Porous Media, Elsevier Publishing, New York, p. 42-54.

Pickens, J.F. (1978): "The Effect of Aquifer Stratification on the Determination of Dispersivity", Presented at the Joint Annual Meeting of Geol. Assoc. Can./Geol. Soc. of Amer., Toronto, Canada, Abstracts and Programs, p. 472.

Pickens, J.F. and Grisak, G.E. (1981a): "Modeling of Scale-Dependent Dispersion in Hyrogeologic Systems", Water Resources Research, Vol. 17, No. 6, p. 1701-1711.

Pickens, J.F. and Grisak, G.E. (1981b): "Scale-Dependent Dispersion in a Stratified Granular Aquifer", Water Resources Research, Vol. 17, No. 4, p. 1191-1211.

Poulin, T.J. (1985): "the Determination of the Coefficient of Dispersion in Sandstones", M.S. Thesis, University of Oklahoma, Norman, Oklahoma.

Prausnitz, J.M. (1958): "Longitudinal Dispersion in a Packed Beds", American Institute of Chemical Engineering Joumal, Vol. 4, No. 1, p. 14M.

Raimondi, P., Gardner, G.H.F., and Patrick, C.B. (1959); "Effect of Pore Structure and Molecular Diffusion on the Mixing of Miscible Liquids Flowing in Porous Media", Reprint 43, Presented at AICHE-SPE Joint Symposium, San Francisco, California.

Raimondi, P., Torcaso, M.A., and Henderson, J.H. (1961): "The effect of Interstitial Water on the Mixing of Hydrocarbons During a Miscible Displacement Process", Mineral Industrial Experiment station Circular, No. 61, Pennsylvania State University.

Rink, M. and Schopper, J.R. (1974): "Interface Conductivity and Its Implications to Electric Logging", Presented at the SPWLA 15th Annual Logging Symposium, Paper J.

Rust, C.F. (1952): "Electrical Resistivity Measurements on Reservoir Rock Samples by the Two-Electrode and four-Electrode Methods", Trans. AMME, Vol. 195, p. 217-224.

Sandrea, R. and Nielsen, R.F. (1974): "Gas-Oil Displacement With Mass Transfer", Dynamics of Petroleum Reservoirs Under Gas Injection, Gulf, Houston, TX., p. 104158.

Scheidegger, A.E. (1954): "Statistical Hydrodynamics in Porous Media", Joumal of Apply Physics, No. 25, p. 994-1001. 
Scheidegger, A.E. (1957): "On the Theory of Flow of Miscible Phases in Porous Media", C.R. Ass. Toronto. Assoc. Int. Hydrol. Scient., Vol. 2, p. 236-242.

Scheidegger, A.E. and Larson, V.C. (1958): "Asymmetry of the Concentration Front During Miscible Displacement in Porous Media", Can.Jour. Phys., Vol. 36, p. 14761482.

Scheidegger, A.E. (1961): "General Theory of Dispersion in Porous Media", Jour. of Geophy. Research, Vol. 66, No. 10, p. 3273-3279.

Scheidegger, A.E. (1972): "The Physics of Flow through Porous Media'. 3rd Edition, University of Toronto Press, Canada.

Schlumberger (1990): "Log Interpretation Principles and Applications". Schlumberger Educational Services, Houston, Texas.

Schlumberger (1991): "Log Interpretation Charts, Schlumberger Educational Services", Houston, Texas.

Schwartz, F.W. (1977): "Macroscopic Dispersion in Porous Media: The Controlling Factors", Water Resources Research, Vol. 13, No. 4, p. 743-752.

Serra, O. (1984): "Fundamental of Well-Log Interpretation, : The Acquisition of Logging Data", Developments in Petroleum Science, 15A, Elsevier, New york.

Slawinski, A. (1926) :Jour. Chim Phys, Vol. 23, p. 710.

Slichter, C.S. (1905): "Field Measurements of the Rate of Movement of Underground Water", U.S. Geological Survey Water-Supply, Paper 140, p. 9-85.

Stalkup, F.I. (1970): "Displacement of Oil by Solvent at High Water saturation", Society of Petroleum Engineers Joumal, p. 337-348.

Stalkup, F.I. (1983): Miscible Displacement, SPE Monograph Series, Vol. 8.

Taylor, G. (1953): "Dispersion of Solute Matter in Solvent Flowing Slowly Through a Tube", Proc. Royal Soci., Vol. 219A, p. 186-203.

Taylor, S.R., Moltyaner, G.L., Howard, K.W.F., and Killey, R.W.D. (1987): "A Comparison of Field and Laboratory Methods for determining Contaminant Flow Parameters", Ground Water, Vol. 25, No. 3, p. 321-330. 
Thesis, C.V. (1962): "Notes on Dispersion in Fluid Flow by Geologic Features", in Morgan, J.M., Jamison, D.K., and Stevenson, J.D., Bditors, U.S.Atomic Energy Commission, TID-7628, p. 166-178.

Thomas, G.H., Countryman, G.R., and Fatt, I. (1963): "Miscible Displacement in a Multiphase System", Society of Petroleum Engineers Joumal, p. 189-196.

Timur, A. (1968): "An investigation of Permeability, Porosity, and Residual Water Saturation Relationships for Sandstone Reservoirs", The Log Analyst, Vol. 9, No. 4, p. 8-17.

Turner, G.A. (1958): "The Flow-Structure in Packed Beds: A Theoretical Investigation Utilizing Frequency Response", Chem. Eng. Sci, Vol. 7, No. 3, p. 156-165.

Walsh, J.B. and Brace, W.F. (1984): "The Effect of Pressure on Porosity and the Transport Properties of Rock", Joumal of Geophys. Res., Vol. 89, No. 11, p. 94259431.

Winsauer, W.O., Shearin, H.M., Jr., Masson, P.H., and Williams, M. (1952): "Resistivity of Brine-Saturated Sands in Relation to Pore Geometry", AAPG Bull, Vol. 36, No. 2, p. 253-277.

Winsauer, W.O. and McCardell, W.M. (1953): "Ionic Double-Layer Conductivity in Reservoir Rock", Trans. ATME, Vol. 198, p. 129-134.

Wong, P.Z. (1988): "The Statistical of Sedimentary Rocks", Physics Today, December, p. 24-32.

Wyllie, M.R.J. and Rose, W. (1950): "Some Theoretical Considerations Related to the Quantitative Evaluation of the Physical Characteristics of Reservoir Rock from Electrical log Data", Joumal of Petroleum Technology, Vol. 2, No. 4, p. 105-118.

Wyllie, M.R.J. and Spangler, M.D. (1952): "Application of Electrical Resistivity Measurements to Problems of Fluid Flow in Porous Media", AM. Assoc. Pet. Geol. Bull, Vol. 36, No. 2, p. 359-403.

Wyllie, M.R.J. and Gregory, A.R. (1955): "Fluid Flow Through Unconsolidated Porous Aggregates", Ind. Eng. Chem., Vol. 47, No. 7, p. 1379-1388.

Yelling, W.F. and Baker, L.E. (1981): "Factors Affecting Miscible Flooding Dispersion Coefficients", Joumal of Canadian. Petroleum Technology, October-December, p. 69-75. 

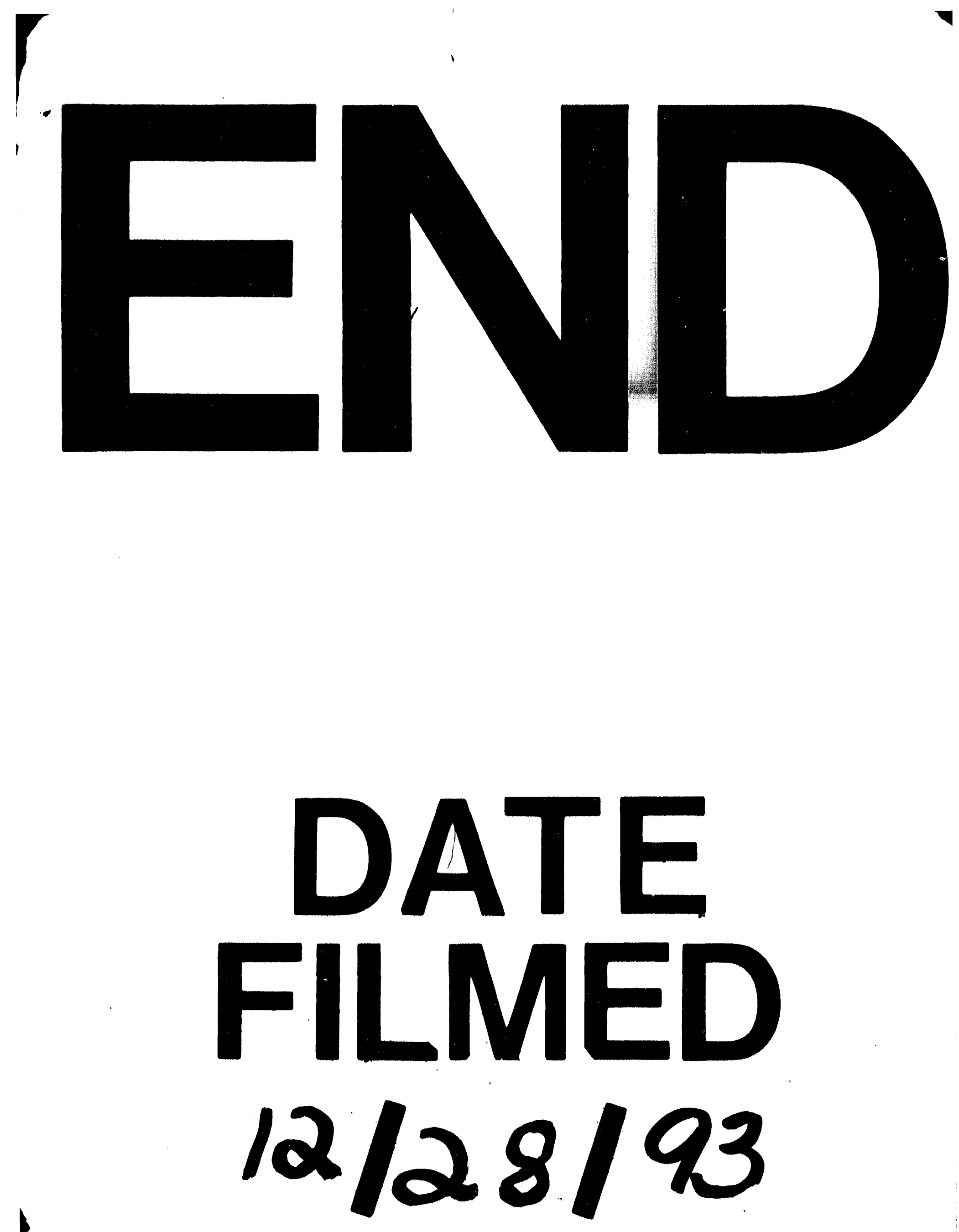


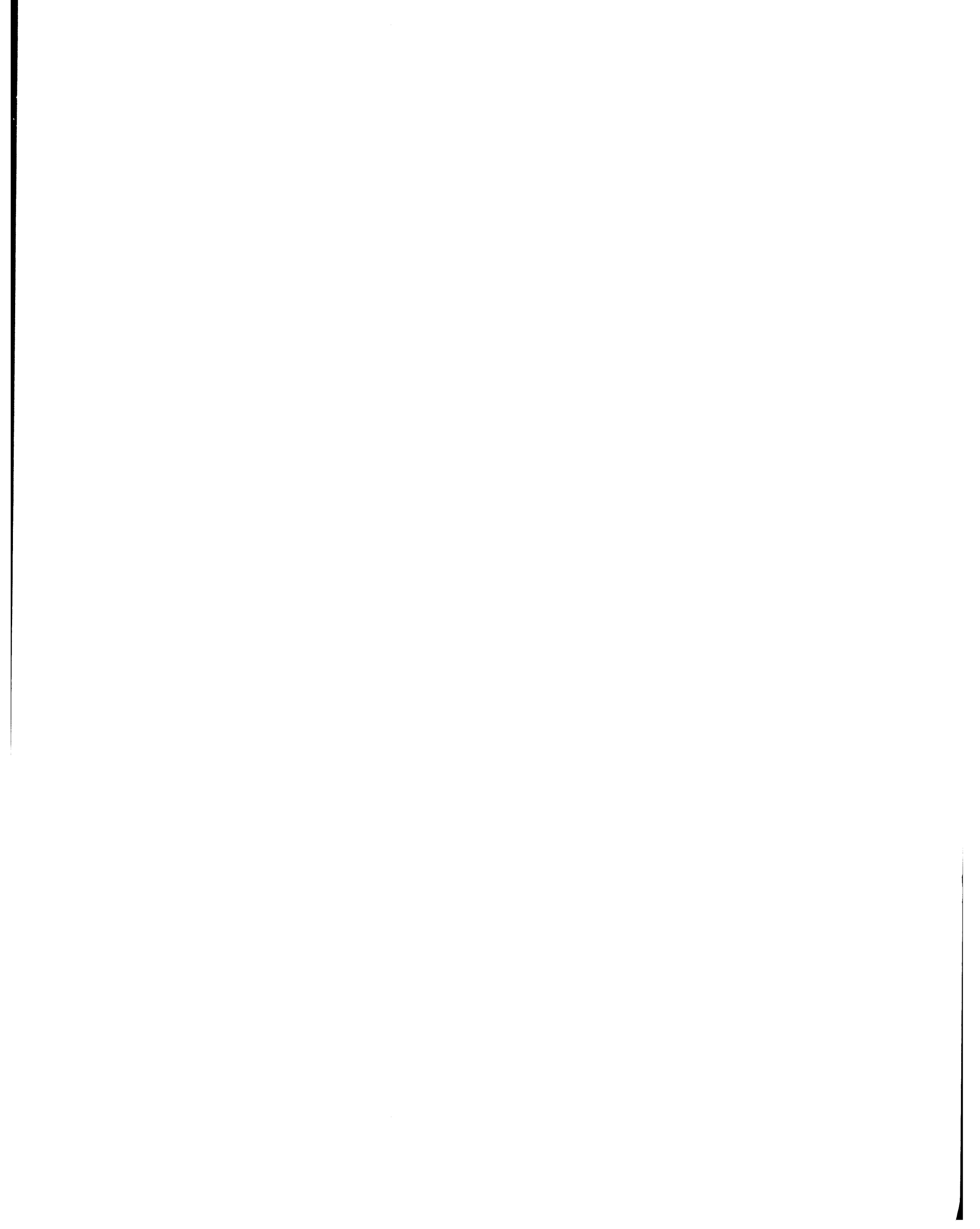

